\title{
Promiscuous installation of D-amino acids in gene-encoded peptides
}

\author{
Madlen Korneli, Sebastian W. Fuchs, Katja Felder, Chantal Ernst, Léa V. Zinsli, and Jörn Piel*
}

\begin{abstract}
D-amino acids can have major effects on the structure, proteolytic stability, and bioactivity of peptides. Proteusin radical $S$ adenosyl methionine epimerases regioselectively install such residues in ribosomal peptides to generate peptides with the largest number of D-residues currently known in biomolecules. To study their utility in synthetic biology, we investigated the substrate tolerance and substrateproduct relationships of the cyanobacterial model epimerase OspD using libraries of point mutants as well as distinct extended peptides that were fused to an $\mathrm{N}$-terminal leader sequence. OspD was found to exhibit exceptional substrate promiscuity in E. coli, accepting 15 different amino acids and converting peptides with a broad range of compositions, secondary structures, and polarities. Diverse single and multiple epimerization patterns were identified that were dictated by the peptide sequence. The data suggest major potential in creating genetically encoded products previously inaccessible by synthetic biology.
\end{abstract}




\section{Table of Contents}

Experimental Procedures

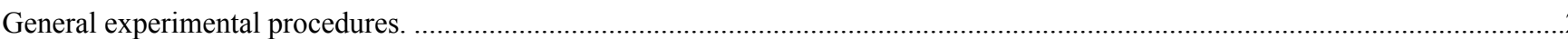

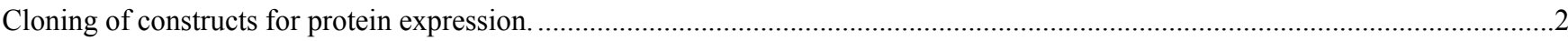

Protein expression of unlabeled precursor proteins and orthogonal $\mathrm{D}_{2} \mathrm{O}$-based induction system (ODIS) for labeling epimerized

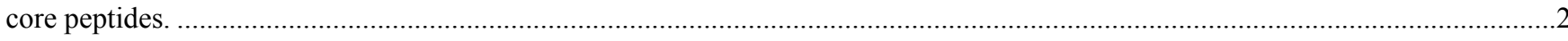

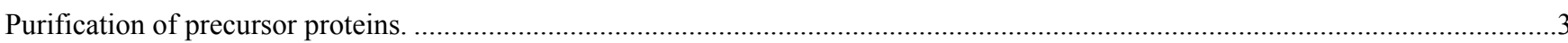

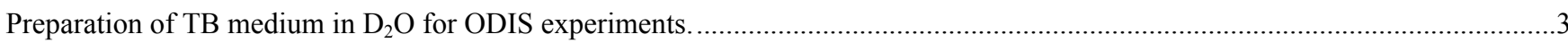

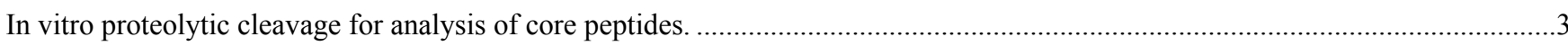

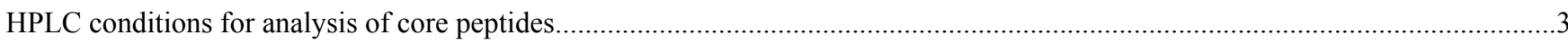

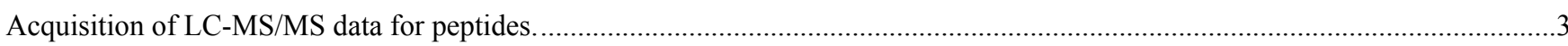

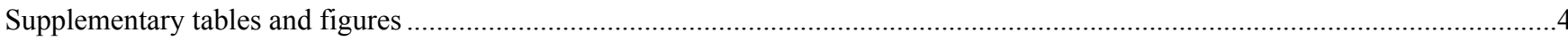

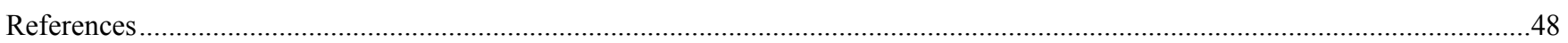

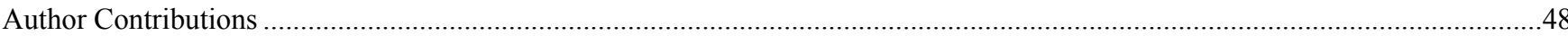

\section{Experimental Procedures}

\section{General experimental procedures}

E. coli DH5 $\alpha$ (Invitrogen) was used for plasmid maintenance and DNA manipulations. All reagents were used according to the manufacturer's instructions. Unless stated otherwise, chemicals were purchased from Sigma Aldrich. Exceptions are isopropyl $\beta$-D-1thiogalactopyranoside (IPTG) from Biosynth and dithiothreitol (DTT) from Applichem. NucleoSpin ${ }^{\mathrm{TM}}$ Plasmid and NucleoSpin ${ }^{\mathrm{TM}}$ Gel and PCR Clean-up kits were obtained from Macherey-Nagel. All PCR primers were ordered from Microsynth. Phusion ${ }^{\mathrm{TM}}$ polymerase came from Thermo Scientific. DpnI restriction enzyme and Gibson Assembly ${ }^{\circledR}$ master mix were from New England Biolabs. Yeast Extract and Tryptone were purchased from Oxoid. Selective antibiotics were obtained from Applichem and used at the following concentrations: 40 $\mu \mathrm{g} / \mathrm{mL}$ kanamycin sulfate (Kan), $100 \mu \mathrm{g} / \mathrm{mL}$ ampicillin sodium salt (Amp), and $40 \mu \mathrm{g} / \mathrm{mL}$ spectinomycin dihydrochloride 5-hydrate (Spt). Protino ${ }^{\circledR}$ Ni-NTA Agarose was purchased from Macherey-Nagel and Factor Xa protease was from Merck. Deuterium oxide (99.90\% D) used in labeling experiments was purchased from Euriso-top. LC-MS experiments were performed on a Dionex Ultimate 3000 UHPLC coupled to a Thermo Scientific Q Exactive mass spectrometer Hybrid Quadrupole-Orbitrap Mass Spectrometer using heated electrospray ionization in positive ion mode. Solvents were Optima ${ }^{\text {TM }}$ LC-MS grade from Fisher Scientific, and the formic acid additive was from Carl Roth.

\section{Cloning of constructs for protein expression}

His $_{6}$-OspA-Fx-I86 and -V95 core mutants, respectively, were generated according to the modified "exponential" QuikChange protocol using pET-28b_ospA-Fx as a template. ${ }^{l}$ Primers were designed with an overlapping region with mutations only on one primer that allowed for exponential amplification of the full plasmid, providing homologous regions for plasmid circularization by Gibson ligation (Table S1).

Nucleotide sequences encoding non-native core peptides were designed for heterologous expression in E. coli from the peptide primary structures using the codon optimization tool of integrated DNA technologies (http://eu.idtdna.com/CodonOpt).

Using pET-28b_ospA-Fx and pCDFDuet_ospA-Fx, respectively, as template, primer pairs were designed to generate His 6 -OspA-Fx-point mutation and non-native core mutants (Table S1). In two separate PCRs, two complementary PCR products were generated each carrying half of the plasmid and half of the corresponding insert with appropriate homologous overlapping regions. ${ }^{2}$ Vectors were assembled via Gibson Assembly ${ }^{\circledR}$ and screened with E. coli DH5 $\alpha$. For all generated constructs, the protein-coding region was confirmed by DNA sequencing (Microsynth). Strains were maintained as $-80^{\circ} \mathrm{C}$ cell stocks prepared from fresh overnight cultures, pelleted and resuspended in $0.5 \times$ LB medium and $25 \%$ glycerol.

Protein expression of unlabeled precursor proteins and orthogonal $\mathrm{D}_{2} \mathrm{O}$-based induction system (ODIS) for labeling epimerized core peptides

Production of His6-OspA-Fx-core mutants was performed as previously described with minor changes. ${ }^{3}$ In brief, NHis precursor proteins encoded in pET-28b and pCDF-Duet, respectively, were co-transformed with epimerase construct in pBAD_Myc-HisA into E. coli BL21(DE3) (Table S2). ${ }^{4}$ The production of unmodified precursor protein was induced with $0.1 \mathrm{mM}$ IPTG. The cells grown in $30 \mathrm{~mL}$ TB medium for 16 hours at $16^{\circ} \mathrm{C}(250 \mathrm{rpm})$. The cell pellet of a $10 \mathrm{~mL}$ aliquot containing the unmodified precursor proteins were flash frozen in liquid nitrogen and kept at $-80^{\circ} \mathrm{C}$. The remaining $20 \mathrm{~mL}$ were centrifuged $(10$ minutes, $3220 \times \mathrm{g})$, supernatant removed and rinsed with TB medium $(2 \times 30 \mathrm{~mL})$ to remove residual IPTG. The rinsed cells were divided into two parts and centrifuged. The first aliquot of cell pellets was reconstituted in TB medium $(20 \mathrm{~mL})$ containing appropriate antibiotics and $0.2 \%(\mathrm{w} / \mathrm{v})$ L-arabinose, whereas the second part 
was reconstituted in $18-20 \mathrm{~mL}$ TB with antibiotics and $0.2 \%(\mathrm{w} / \mathrm{v})$ L-arabinose all resolved in $\mathrm{D}_{2} \mathrm{O}$. Cell cultures were grown in $50 \mathrm{~mL}$ Falcon tubes for 24 hours $\left(16^{\circ} \mathrm{C}, 250 \mathrm{rpm}\right)$. The cells were collected $(3220 \times \mathrm{g}, 10$ minutes $)$ and subjected to protein purification in parallel with the first sample only induced with IPTG.

\section{Purification of precursor proteins}

Unmodified, epimerized and labeled precursor proteins were purified as previously reported. ${ }^{3}$ Minor changes were: cell lysis was accomplished by sonication using a Q700 Sonicator (Qsonica) with a $10 \times 10 \mathrm{sec}$ on/off each pulse sequence and a $6.4 \mathrm{~mm}$ probe at $40 \%$ amplitude. Cell lysates were clarified by centrifugation at $3220 \times \mathrm{g}$ for $80 \mathrm{~min}$. $\mathrm{Ni}^{2+}$ - bound NHis precursor proteins were eluted with 100 $\mu \mathrm{L}$ elution buffer $(50 \mathrm{mM}$ sodium phosphate, $300 \mathrm{mM}$ sodium chloride, $10 \%$ [v/v] glycerol, $250 \mathrm{mM}$ imidazole, $\mathrm{pH} 8.0)$ and subjected directly to in vitro cleavage.

\section{Preparation of TB medium in $\mathrm{D}_{2} \mathrm{O}$ for ODIS experiments}

$1 \mathrm{~L}$ TB medium in $\mathrm{D}_{2} \mathrm{O}$ was produced by first dissolving $12 \mathrm{~g}$ tryptone and $24 \mathrm{~g}$ yeast extract in $900 \mathrm{~mL}_{2} \mathrm{O}$. Secondly, the supplementary potassium phosphate solution was produced by dissolving $2.31 \mathrm{~g} \mathrm{KH}_{2} \mathrm{PO}_{4}(0.17 \mathrm{M})$ and $12.54 \mathrm{~g} \mathrm{~K}_{2} \mathrm{HPO}_{4}(0.72 \mathrm{M})$ in $100 \mathrm{~mL} \mathrm{D} \mathrm{O}$. A $20 \%$ $(\mathrm{w} / \mathrm{v})$ of L-arabinose solution $\left(2 \mathrm{~g}\right.$ in $\left.10 \mathrm{~mL} \mathrm{D}_{2} \mathrm{O}\right)$ was used for induction. All solutions were filter sterilized $(0.22 \mu \mathrm{m})$ and mixed freshly for each ODIS experiment.

\section{In vitro proteolytic cleavage for analysis of core peptides}

Typically, $20 \mu \mathrm{L}$ of elution fraction of unmodified, epimerized or labeled precursor proteins was used for cleavage. To this protein solution, $68 \mu \mathrm{L} \mathrm{dH} \mathrm{d}_{2} \mathrm{O}, 10 \mu \mathrm{L}$ Factor Xa buffer (200 mM Tris, $500 \mathrm{mM} \mathrm{NaCl}, 20 \mathrm{mM} \mathrm{CaCl}_{2}, \mathrm{pH}$ 8.0), $2 \mu \mathrm{L}$ of Factor Xa protease and $1.5 \mathrm{mM}$ DTT were added. The mixture was incubated at room temperature overnight and subsequently analyzed by LC-MS. In addition, trypsin was used

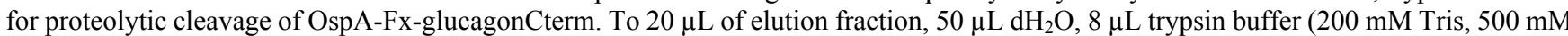
$\mathrm{NaCl}, 20 \mathrm{mM} \mathrm{CaCl}_{2}, \mathrm{pH} 8.0$ ), $2 \mu \mathrm{L}$ of trypsin solution (dissolved in $50 \mathrm{mM}$ acetic acid, $0.5 \mu \mathrm{g} / \mathrm{mL}$ ), and $1.0 \mathrm{mM}$ DTT were added. The mixture was incubated at $37^{\circ} \mathrm{C}$ for $3 \mathrm{~h}$ and subsequently analyzed by LC-MS.

\section{HPLC conditions for analysis of core peptides}

The mixture of the proteolytic digest $(5 \mu \mathrm{L})$ was analyzed by reversed phase HPLC. HPLC conditions for OspA point mutants and truncated variants, A $\beta 10-23$, protegrin as well as GnRH-G6S: column: Phenomenex, Kinetex, C18-XB, $2.6 \mu \mathrm{m}, 150 \times 4.6 \mathrm{~mm}$; column temperature: $50{ }^{\circ} \mathrm{C}$; flow rate $1 \mathrm{~mL} / \mathrm{min}$; mobile phase/gradient: $10 \% \mathrm{CH}_{3} \mathrm{CN}+0.1 \%$ formic acid $/ \mathrm{H}_{2} \mathrm{O}+0.1 \%$ formic acid for $0.5 \mathrm{~min}$ then ramped to $40 \% \mathrm{CH}_{3} \mathrm{CN}+0.1 \%$ formic acid $/ \mathrm{H}_{2} \mathrm{O}+0.1 \%$ formic acid over $11.5 \mathrm{~min}$.

HPLC conditions for split GFP: column: Phenomenex, Kinetex, C18-XB, $2.6 \mu \mathrm{m}, 150 \times 4.6 \mathrm{~mm}$; column temperature: $50^{\circ} \mathrm{C}$; flow rate 1 $\mathrm{mL} / \mathrm{min}$; mobile phase/gradient: $25 \% \mathrm{CH}_{3} \mathrm{CN}+0.1 \%$ formic acid $/ \mathrm{H}_{2} \mathrm{O}+0.1 \%$ formic acid for 0.5 min then ramped to $60 \% \mathrm{CH}_{3} \mathrm{CN}+0.1 \%$ formic acid $/ \mathrm{H}_{2} \mathrm{O}+0.1 \%$ formic acid over $11.5 \mathrm{~min}$.

HPLC conditions for glucagon ${ }_{1-10}$ : column: Phenomenex, Kinetex, $\mathrm{C} 18-\mathrm{XB}, 2.6 \mu \mathrm{m}, 150 \times 4.6 \mathrm{~mm}$; column temperature: $50^{\circ} \mathrm{C}$; flow rate 1 $\mathrm{mL} / \mathrm{min}$; mobile phase/gradient: $10 \% \mathrm{CH}_{3} \mathrm{CN}+0.1 \%$ formic acid $/ \mathrm{H}_{2} \mathrm{O}+0.1 \%$ formic acid for 0.5 min then ramped to $45 \% \mathrm{CH}_{3} \mathrm{CN}+0.1 \%$ formic acid $/ \mathrm{H}_{2} \mathrm{O}+0.1 \%$ formic acid over $11.5 \mathrm{~min}$.

HPLC conditions for glucagon ${ }_{10-29}$ : column: Phenomenex, Kinetex, $\mathrm{C} 18-\mathrm{XB}, 2.6 \mu \mathrm{m}, 150 \times 4.6 \mathrm{~mm}$; column temperature: $50^{\circ} \mathrm{C}$; flow rate $1 \mathrm{~mL} / \mathrm{min}$; mobile phase/gradient: $5 \% \mathrm{CH}_{3} \mathrm{CN}+0.1 \%$ formic acid $/ \mathrm{H}_{2} \mathrm{O}+0.1 \%$ formic acid for $0.5 \mathrm{~min}$ then ramped to $50 \% \mathrm{CH}_{3} \mathrm{CN}+0.1 \%$ formic acid $/ \mathrm{H}_{2} \mathrm{O}+0.1 \%$ formic acid over $11.5 \mathrm{~min}$.

HPLC conditions for A $330-41$ : column: Phenomenex, Kinetex, C18-XB, $2.6 \mu \mathrm{m}, 150 \times 4.6 \mathrm{~mm}$; column temperature: $50^{\circ} \mathrm{C}$; flow rate 1 $\mathrm{mL} / \mathrm{min}$; mobile phase/gradient: $10 \% \mathrm{CH}_{3} \mathrm{CN}+0.1 \%$ formic acid $/ \mathrm{H}_{2} \mathrm{O}+0.1 \%$ formic acid for 0.5 min then ramped to $60 \% \mathrm{CH}_{3} \mathrm{CN}+0.1 \%$ formic acid $/ \mathrm{H}_{2} \mathrm{O}+0.1 \%$ formic acid over $11.5 \mathrm{~min}$.

HPLC conditions for bactenecin-R4A: column: Phenomenex, Kinetex, C18-XB, $2.6 \mu \mathrm{m}, 150 \times 4.6 \mathrm{~mm}$; column temperature: $50^{\circ} \mathrm{C}$; flow rate $1 \mathrm{~mL} / \mathrm{min}$; mobile phase/gradient: $10 \% \mathrm{CH}_{3} \mathrm{CN}+0.1 \%$ formic acid $/ \mathrm{H}_{2} \mathrm{O}+0.1 \%$ formic acid for $0.5 \mathrm{~min}$ then ramped to $50 \% \mathrm{CH}{ }_{3} \mathrm{CN}$ $+0.1 \%$ formic acid $/ \mathrm{H}_{2} \mathrm{O}+0.1 \%$ formic acid over $11.5 \mathrm{~min}$.

\section{Acquisition of LC-MS/MS data for peptides}

Unmodified, epimerized, or labeled core peptide mixtures were subjected to full-scan LC-MS. Full MS was performed at a resolution of 70,000 (AGC target 1e6, maximum IT $100 \mathrm{~ms}$, scan range 300-1250 m/z). Expected masses were calculated using the ChemCalc online tool $^{5}$ and used in an inclusion list to simultaneously acquire MS2 using parallel reaction monitoring (PRM). PRM was performed at a resolution of 17,500 (AGC target 2e5, maximum IT $100 \mathrm{~ms}$, isolation windows in the range of $4.0 \mathrm{~m} / \mathrm{z}$ ). Normalized collision energy (NCE) was adjusted based on the peptide and charge state to optimize fragmentation and ranged from 25-33. Inclusion lists were adjusted for each peptide and included masses for protonated adducts ranging from 0 to up to 4 deuterium incorporations as well as two charge states $(+2$ and $+3)$. $\mathrm{MS}^{2}$ spectra that display "0D", "+1D", "+2D", etc. in parentheses indicate the number of incorporated deuterium atoms (\# of epimerizations) in the fragment. "0D" indicates no deuterium incorporation. 


\section{Supplementary tables and figures}

Table S1. DNA primers used in this study

\begin{tabular}{|c|c|}
\hline Primer name & DNA sequence $5^{\prime}$ to $3^{\prime}$ \\
\hline mutI106rv & $\begin{array}{l}\text { CCAGCAACCACGACCATCGATAGCTTCTAATTGTTCTTCAGATAATTCT } \\
\text { TCTTCAACAGC }\end{array}$ \\
\hline mutI106Hfw & CGATGGTCGTGGTTGCTGGCATGCTGGTAGCCGTGGCTG \\
\hline mutI106Kfw & CGATGGTCGTGGTTGCTGGAAAGCTGGTAGCCGTGGCTG \\
\hline mutI106Efw & CGATGGTCGTGGTTGCTGGGAAGCTGGTAGCCGTGGCTG \\
\hline mutI106Sfw & CGATGGTCGTGGTTGCTGGTCTGCTGGTAGCCGTGGCTG \\
\hline mutI106Qfw & CGATGGTCGTGGTTGCTGGCAGGCTGGTAGCCGTGGCTG \\
\hline mutI106Gfw & CGATGGTCGTGGTTGCTGGGGCGCTGGTAGCCGTGGCTG \\
\hline mutI106Pfw & CGATGGTCGTGGTTGCTGGCCAGCTGGTAGCCGTGGCTG \\
\hline mutI106Mfw & CGATGGTCGTGGTTGCTGGATGGCTGGTAGCCGTGGCTG \\
\hline mutI106Ffw & CGATGGTCGTGGTTGCTGGTTTGCTGGTAGCCGTGGCTG \\
\hline mutI106Wfw & CGATGGTCGTGGTTGCTGGTGGGCTGGTAGCCGTGGCTG \\
\hline mutV115rv & AAAACCGCAGCCACGGCTACCAGCAATCCAGCAACCACGACC \\
\hline mutV115Hfw & GTAGCCGTGGCTGCGGTTTTCATACTCGCACTTAAAAGCTTGCGGC \\
\hline mutV115Kfw & GTAGCCGTGGCTGCGGTTTTAAAACTCGCACTTAAAAGCTTGCGGC \\
\hline mutV115Efw & GTAGCCGTGGCTGCGGTTTTGAAACTCGCACTTAAAAGCTTGCGGC \\
\hline mutV115Sfw & GTAGCCGTGGCTGCGGTTTTTCTACTCGCACTTAAAAGCTTGCGGC \\
\hline mutV115Qfw & GTAGCCGTGGCTGCGGTTTTCAGACTCGCACTTAAAAGCTTGCGGC \\
\hline mutV115Gfw & GTAGCCGTGGCTGCGGTTTTGGCACTCGCACTTAAAAGCTTGCGGC \\
\hline mutV115Pfw & GTAGCCGTGGCTGCGGTTTTCCAACTCGCACTTAAAAGCTTGCGGC \\
\hline mutV115Mfw & GTAGCCGTGGCTGCGGTTTTATGACTCGCACTTAAAAGCTTGCGGC \\
\hline mutV115Ffw & GTAGCCGTGGCTGCGGTTTTTTCACTCGCACTTAAAAGCTTGCGGC \\
\hline mutV115Wfw & GTAGCCGTGGCTGCGGTTTTTGGACTCGCACTTAAAAGCTTGCGGC \\
\hline OspA_W85A_1 & GCTATTGCTGGTAGCCGTGGCTGCGGTTTTGTAACTCG \\
\hline OspA_W85A_i & ACGGCTACCAGCAATAGCGCAACCACGACCATCGATAG \\
\hline OspA_W85P_1 & CCTATTGCTGGTAGCCGTGGCTGCGGTTTTGTAACTCG \\
\hline OspA_W85P_i & ACGGCTACCAGCAATAGGGCAACCACGACCATCGATAG \\
\hline OspA_W85G_1 & GGTGGCTGGATTGCTGGTAGCCGTGGCTGCGGTTTTG \\
\hline OspA_W85G_i & ACCAGCAATCCAGCCACCACGACCATCGATAGCTTCTAATTGTTCTTC \\
\hline OspA_A87G_1 & GGTGGTAGCCGTGGCTGCGGTTTTGTAACTCGCACTTAAAAG \\
\hline OspA_A87G_i & GCAGCCACGGCTACCACCAATCCAGCAACCACGACC \\
\hline OspA_A87P_1 & ATTCCTGGTAGCCGTGGCTGCGGTTTTGTAACTCGCACTTAAAAG \\
\hline OspA_A87P_i & GCCACGGCTACCAGGAATCCAGCAACCACGACCATCGATAG \\
\hline OspA_F94A_1 & GCTGTAACTCGCACTTAAAAGCTTGCGGCCGCACTC \\
\hline OspA_F94A_i & TTAAGTGCGAGTTACAGCACCGCAGCCACGGCTACCAG \\
\hline OspA_F94P_1 & CCTGTAACTCGCACTTAAAAGCTTGCGGCCGCACTCG \\
\hline OspA_F94P_i & TTAAGTGCGAGTTACAGGACCGCAGCCACGGCTACCAG \\
\hline OspA_F94G_1 & GGCGTAACTCGCACTTAAAAGCTTGCGGCCGCAC \\
\hline
\end{tabular}




\begin{tabular}{|c|c|}
\hline OspA_F94G_i & TTAAGTGCGAGTTACGCCACCGCAGCCACGGCTAC \\
\hline OspA_T96A_1 & TTTGTAGCTCGCACTTAAAAGCTTGCGGCCGCACTC \\
\hline OspA_T96A_i & TTAAGTGCGAAGCACAAAACCGCAGCCACGGCTACCAGC \\
\hline OspA_T96G_1 & TTTGTAGGCCGCACTTAAAAGCTTGCGGCCGCACTC \\
\hline OspA_T96G_i & TTAAGTGCGAGCCACAAAACCGCAGCCACGGCTACCAGC \\
\hline OspA_T96P_1 & TTTGTACCTCGCACTTAAAAGCTTGCGGCCGCACTC \\
\hline OspA_T96P_i & TTAAGTGCGATGGACAAAACCGCAGCCACGGCTACCAGCAATC \\
\hline OspA_88V91V93I_1 & TAGCCGTGTATGCATCTTTGTAACTCGCACTTAAAAGCTTGC \\
\hline OspA_G83IG88V_i & $\begin{array}{l}\text { AAAAATGCATACACGGCTAACAGCAATCCAGCAGATACGACCATCGAT } \\
\text { AG }\end{array}$ \\
\hline C112X_Fwd & GGATTGCTGGTAGCCGTGGCTAAGGTTTTGTAACTCGCACTTAAA \\
\hline C112X_Rev & GCCACGGCTACCAGCAATCCAGCAACCACGACCATCGATA \\
\hline S109X_Fwd & GTGGTTGCTGGATTGCTGGTTAACGTGGCTGCGGTTTTGTAACTC \\
\hline S109X_Rev & ACCAGCAATCCAGCAACCACGACCATCGATAGCTTCTAATTGTTC \\
\hline OspA_GnRH-G6S_1 & AGTCTGCGCCCTGGATAAAAGCTTGCGGCCGCAC \\
\hline OspA_GnRH-G6S_i & $\begin{array}{l}\text { TTATCCAGGGCGCAGACTATAAGACCAATGCTCACGACCATCGATAGC } \\
\text { TTC }\end{array}$ \\
\hline beta_amy1023_1 & CACCAGAAATTAGTGTTCTTTGCGGAGGACTAAAAGCTTGCGGCC \\
\hline beta_amy1023_i & GAACACTAATTTCTGGTGGTGTACCTCATAACGACCATCGATAGCTTC \\
\hline beta_30-41_1 & $\begin{array}{l}\text { GATTGATGGTCGGCGGAGTAGTCATTTAAAAGCTTGCGGCCGCATAAT } \\
\text { G }\end{array}$ \\
\hline beta_30-41_i & CTCCGCCGACCATCAATCCAATAATCGCACGACCATCGATAGCTTC \\
\hline OspA_ProtI_1 & $\begin{array}{l}\text { GTCGTCGTCGCTTCTGTGTGTGTGTAGGGCGTTAAAAGCTTGCGGCCGC } \\
\text { AC }\end{array}$ \\
\hline OspA_ProtI_i & $\begin{array}{l}\text { CACAGAAGCGACGACGACAATAACAAAGACGCCCGCCACGACGACCA } \\
\text { TCGATAGC }\end{array}$ \\
\hline Split_GFP11_1 & $\begin{array}{l}\text { CCACATGGTGCTGCTTGAGTTTGTAACTGCTGCTGGGATTACATAAAAG } \\
\text { CTTGCGGCCG }\end{array}$ \\
\hline Split_4LinkGFP11_i & $\begin{array}{l}\text { TCAAGCAGCACCATGTGGTCACGGCTGCCGTCGCCACGACCATCGATA } \\
\text { GCTTCTAATTG }\end{array}$ \\
\hline OspA_Bactenecin_1 & CTATTGTAGTTATTCGCGTTTGTCGCTAAAAGCTTGCGGCCGCACTC \\
\hline OspA_Bactenecin_i & CGCGAATAACTACAATAGCACAAAGACGACGACCATCGATAGCTTC \\
\hline pET28b_ospA_Gib_1 & AGCGAGTCAGTGAGCGAGGAAGCGGAAGAGCG \\
\hline pET28b_ospA_Gib_i & CTCGCTCACTGACTCGCTGCGCTCGGTCGTTC \\
\hline Gib Duet Mid long 1 & GGGACAACGTAAGCACTACATTTCGCTCATCGCCAGC \\
\hline Gib Duet Mid long i & $\begin{array}{l}\text { GTAGTGCTTACGTTGTCCCGCATTTGGTACAGCGCAGTAACCGGCAAAA } \\
\text { TC }\end{array}$ \\
\hline
\end{tabular}

Table S2. Plasmids generated and used in this work.

The numbering is based on the primary structure of the expressed His6-OspA. Fx denotes a factor Xa cleavage site between leader and core.

\begin{tabular}{lll}
\hline Plasmid & Specifications & Reference \\
\hline pET-28b_osp $A$-Fx & KanR, ColE1 (pBR322) & Morinaka et al. ${ }^{6}$ \\
pET-28b_osp $A$-Fx_I86H & KanR, ColE1 (pBR322) & This work \\
pET-28b_osp $A$-Fx_I86K & KanR, ColE1 (pBR322) & This work \\
pET-28b_osp $A$-Fx_I86E & KanR, ColE1 (pBR322) & This work \\
pET-28b_osp $A-F x \_I 86 S$ & KanR, ColE1 (pBR322) & This work
\end{tabular}




\begin{tabular}{|c|c|c|}
\hline pET-28b_ospA-Fx_I86Q & KanR, ColE1 (pBR322) & This work \\
\hline pET-28b_ospA-Fx_I86G & KanR, ColE1 (pBR322) & This work \\
\hline pET-28b_osp $A$-Fx_I86P & KanR, ColE1 (pBR322) & This work \\
\hline pET-28b_ospA-Fx_I86M & KanR, ColE1 (pBR322) & This work \\
\hline pET-28b_osp $A$-Fx_I86F & KanR, ColE1 (pBR322) & This work \\
\hline pET-28b_osp $A$-Fx_I86W & KanR, ColE1 (pBR322) & This work \\
\hline pET-28b_ospA-Fx_V95H & KanR, ColE1 (pBR322) & This work \\
\hline pET-28b_ospA-Fx_V95K & KanR, ColE1 (pBR322) & This work \\
\hline pET-28b_ospA-Fx_V95E & KanR, ColE1 (pBR322) & This work \\
\hline pET-28b_ospA-Fx_V95S & KanR, ColE1 (pBR322) & This work \\
\hline pET-28b_ospA-Fx_V95Q & KanR, ColE1 (pBR322) & This work \\
\hline pET-28b_ospA-Fx_V95G & KanR, ColE1 (pBR322) & This work \\
\hline pET-28b_osp $A$-Fx_V95P & KanR, ColE1 (pBR322) & This work \\
\hline pET-28b-ospA_Fx_V95M & KanR, ColE1 (pBR322) & This work \\
\hline pET-28b_osp $A-\mathrm{Fx} \_V 95 \mathrm{~F}$ & KanR, ColE1 (pBR322) & This work \\
\hline pET-28b_osp $A$-Fx_V95W & KanR, ColE1 (pBR322) & This work \\
\hline pET-28b_ospA-Fx_W85A & KanR, ColE1 (pBR322) & This work \\
\hline pET-28b_ospA-Fx_W85G & KanR, ColE1 (pBR322) & This work \\
\hline pET-28b_ospA-Fx_W85P & KanR, ColE1 (pBR322) & This work \\
\hline pET-28b_ospA-Fx_A87G & KanR, ColE1 (pBR322) & This work \\
\hline pET-28b_ospA-Fx_A87P & KanR, ColE1 (pBR322) & This work \\
\hline pET-28b_osp $A$-Fx_F94A & KanR, ColE1 (pBR322) & This work \\
\hline pET-28b_osp $A$-Fx_F94G & KanR, ColE1 (pBR322) & This work \\
\hline pET-28b_ospA-Fx_F94P & KanR, ColE1 (pBR322) & This work \\
\hline pET-28b_ospA-Fx_T96A & KanR, ColE1 (pBR322) & This work \\
\hline pET-28b_ospA-Fx_T96G & KanR, ColE1 (pBR322) & This work \\
\hline pET-28b_ospA-Fx_T96P & KanR, ColE1 (pBR322) & This work \\
\hline pET-28b_ospA-Fx_G91VG93I & KanR, ColE1 (pBR322) & This work \\
\hline pET-28b_ospA-Fx_C10X & KanR, ColE1 (pBR322) & This work \\
\hline pET-28b_ospA-Fx_S7X & KanR, ColE1 (pBR322) & This work \\
\hline pCDF-Duet_ospA-Fx & SpeR, CloDF13 & Morinaka et al. ${ }^{6}$ \\
\hline pCDF-Duet_ospA-Fx_glucagonNterm ${ }_{1-10}$ & SpeR, CloDF13 & This work \\
\hline pCDF-Duet_ospA-Fx_glucagonCterm $10-29$ & SpeR, CloDF13 & This work \\
\hline pCDF-Duet_ospA-Fx_GnrH-G6S & SpeR, CloDF13 & This work \\
\hline pCDF-Duet_ospA-Fx_A $\beta 10-23$ & SpeR, CloDF13 & This work \\
\hline pCDF-Duet_ospA-Fx_A $\beta 30-41$ & SpeR, CloDF13 & This work \\
\hline pCDF-Duet_osp $A$-Fx_protegrinI & SpeR, CloDF13 & This work \\
\hline pCDF-Duet_ospA-Fx_bactenecin-R4A & SpeR, CloDF13 & This work \\
\hline $\begin{array}{l}\text { pCDF-Duet_ospA- } \\
\text { Fx_4linker_splitGFP11 }\end{array}$ & SpeR, CloDF13 & This work \\
\hline pBAD-Myc-HisA_ospD & AmpR, AraBAD & Morinaka et al. ${ }^{3}$ \\
\hline
\end{tabular}


A

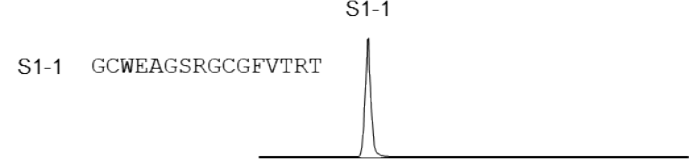

$\mathrm{C}\left[\mathrm{M}+2 \mathrm{H}^{+}\right]^{2+}$
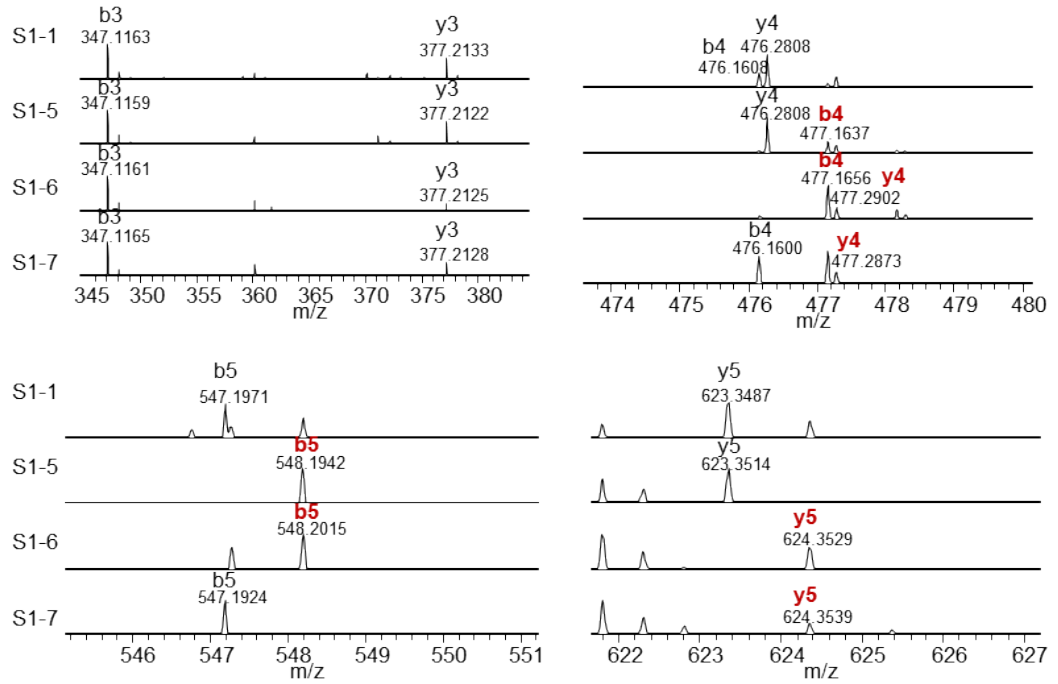

B

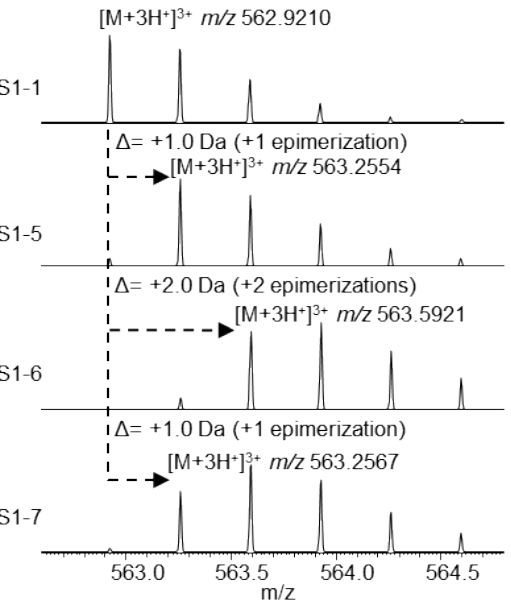

Figure S1. Characterization of selectively labeled products from co-expression of $o s p A-I 4 E+o s p D$ using ODIS. His 6 -OspA variant was cleaved with Factor Xa. (A) EICs of unmodified core peptides (S1-1), core peptides epimerized in TB (S1-2 to S1-4), and epimerized in $\mathrm{D}_{2} \mathrm{O}$-based medium (S1-5 to S1-7). (B) MS for parent ions of S1-1, S1-5 to S1-7 acquired by LC-MS full-scan of ODIS experiment. (C) MS $^{2}$ derived from parent ions of S1-1, S1-5 to S1-7 acquired by LC-MS2 (PRM) of ODIS experiments. Bold (red) residues indicate epimerized residues. Small letter (red) indicates selective labeling with deuterium at the corresponding epimerized residue. 


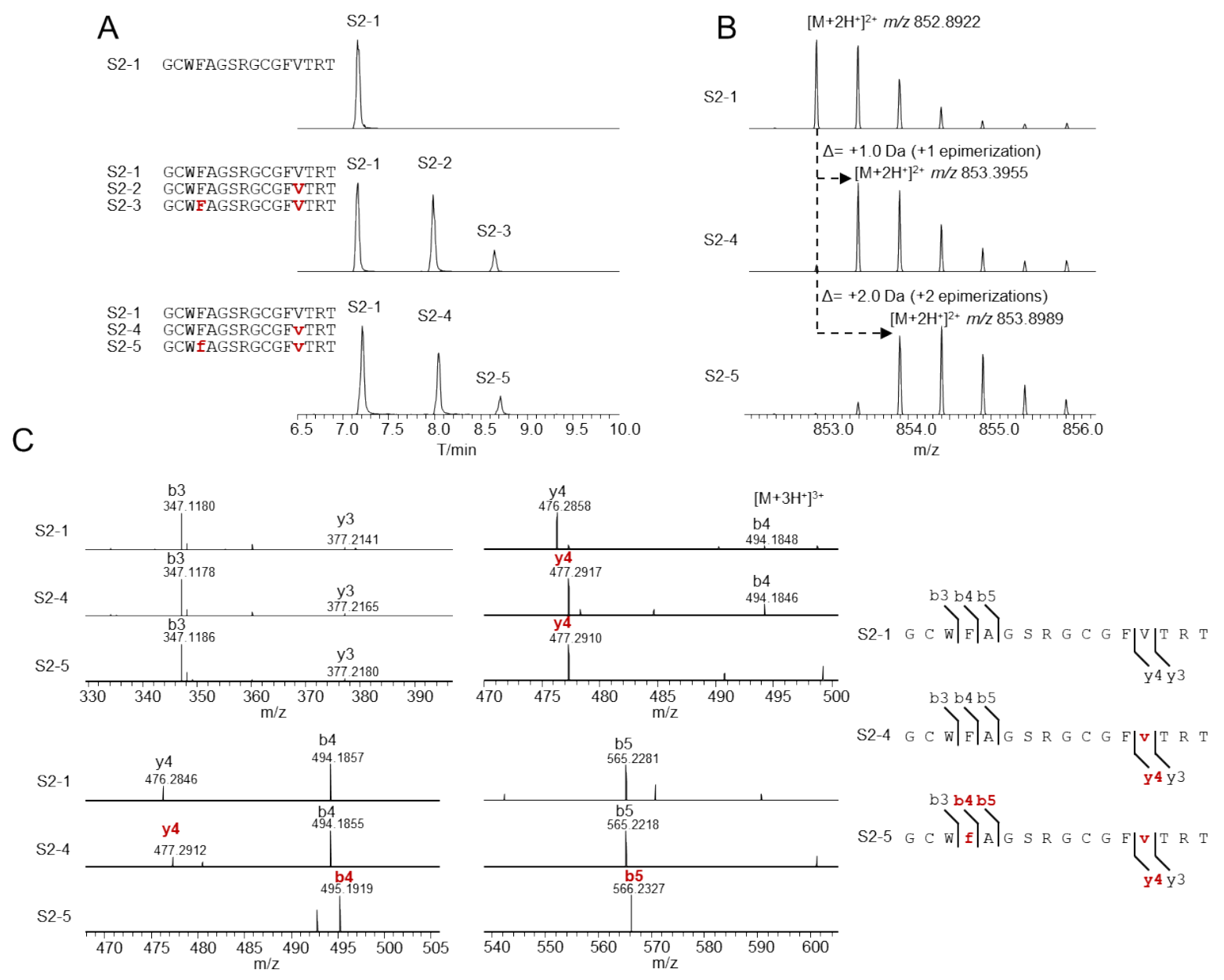

Figure S2. Characterization of selectively labeled products from co-expression of $o s p A-I 4 F+o s p D$ using ODIS. His ${ }_{6}$-OspA variant was cleaved with Factor Xa. (A) EICs of unmodified core peptides (S2-1), core peptides epimerized in TB (S2-2 to S2-3), and epimerized in $\mathrm{D}_{2} \mathrm{O}$-based medium (S2-4 to S2-5). (B) MS for parent ions of S2-1, S2-4 to S2-5 acquired by LC-MS full-scan of ODIS experiment. (C) MS $^{2}$ derived from parent ions of S2-1, S2-4 to S2-5 acquired by LC-MS 2 (PRM) of ODIS experiments. Bold (red) residues indicate epimerized residues. Small letter (red) indicates selective labeling with deuterium at the corresponding epimerized residue. 


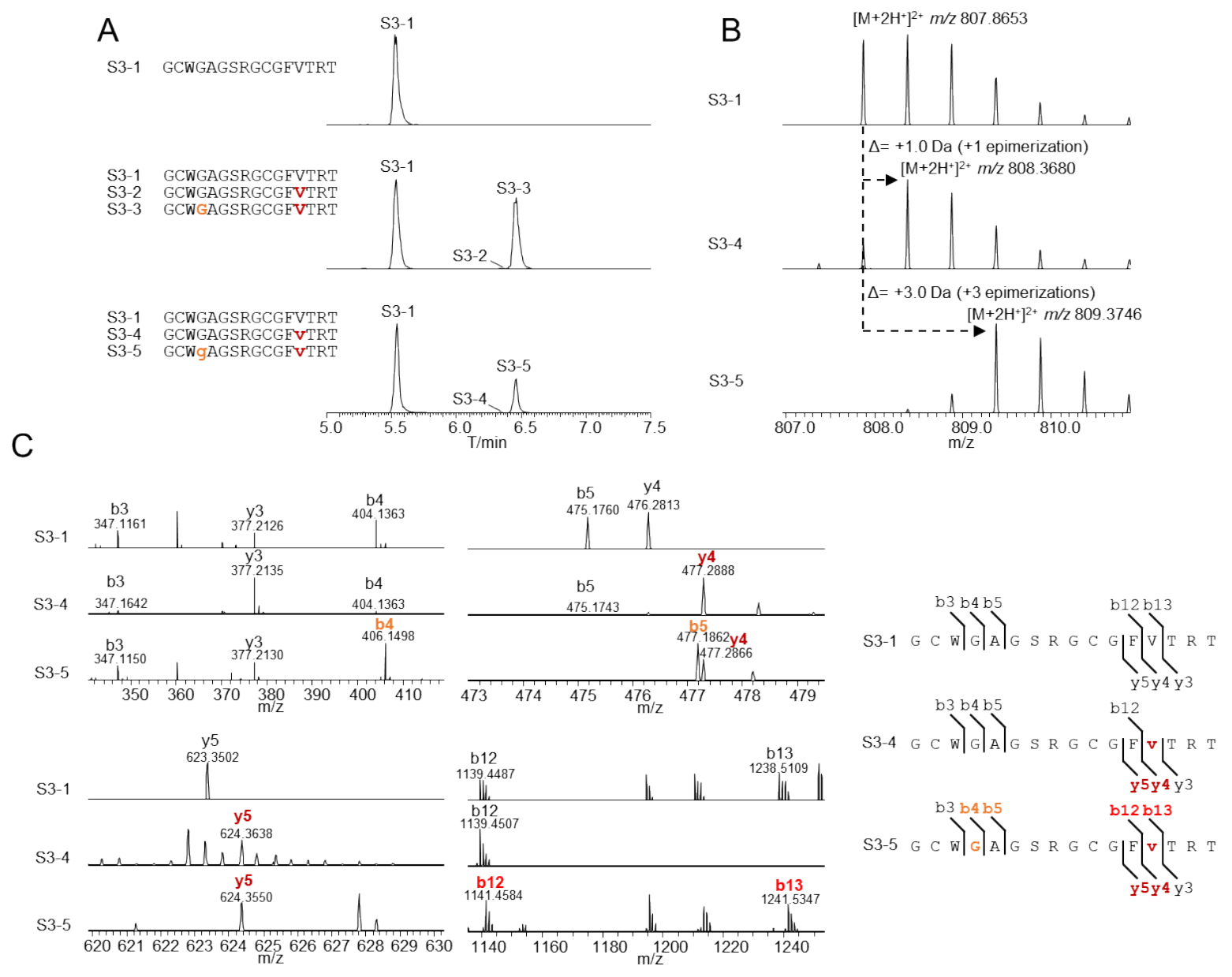

Figure S3. Characterization of selectively labeled products from co-expression of $o s p A-I 4 G+o s p D$ using ODIS. His 6 -OspA variant was cleaved with Factor Xa. (A) EICs of unmodified core peptides (S3-1), core peptides epimerized in TB (S3-2 to S2-3), and epimerized in $\mathrm{D}_{2} \mathrm{O}$-based medium (S3-4 to S3-5). (B) MS for parent ions of S3-1, S3-4 to S3-5 acquired by LC-MS full-scan of ODIS experiment. (C) $\mathrm{MS}^{2}$ derived from parent ions of S3-1, S3-4 to S3-5 acquired by LC-MS 2 (PRM) of ODIS experiments. Bold (red) residues indicate epimerized residues. Bold (orange) Gly indicates bideuterated residue. Small letter (red) indicates selective labeling with deuterium at the corresponding epimerized residue. 


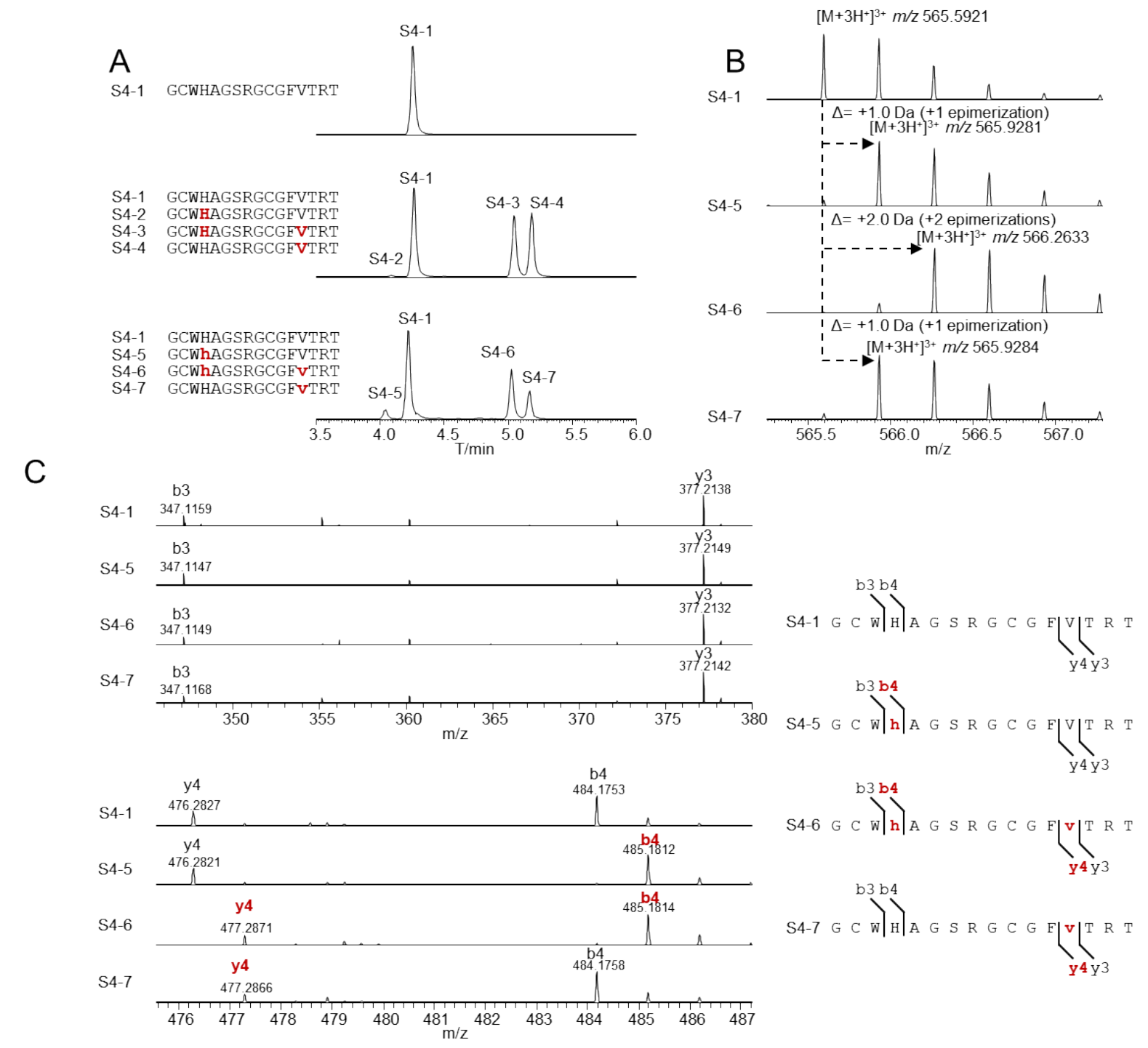

Figure S4. Characterization of selectively labeled products from co-expression of $o s p A-I 4 H+o s p D$ using ODIS. His ${ }_{6}$-OspA variant was cleaved with Factor Xa. (A) EICs of unmodified core peptides (S4-1), core peptides epimerized in TB (S4-2 to S4-4), and epimerized in $\mathrm{D}_{2} \mathrm{O}$-based medium (S4-5 to S4-7). (B) MS for parent ions of S4-1, S4-5 to S4-7 acquired by LC-MS full-scan of ODIS experiment. (C) MS $^{2}$ derived from parent ions of S4-1, S4-5 to S4-7 acquired by LC-MS 2 (PRM) of ODIS experiments. Bold (red) residues indicate epimerized residues. Small letter (red) indicates selective labeling with deuterium at the corresponding epimerized residue. 


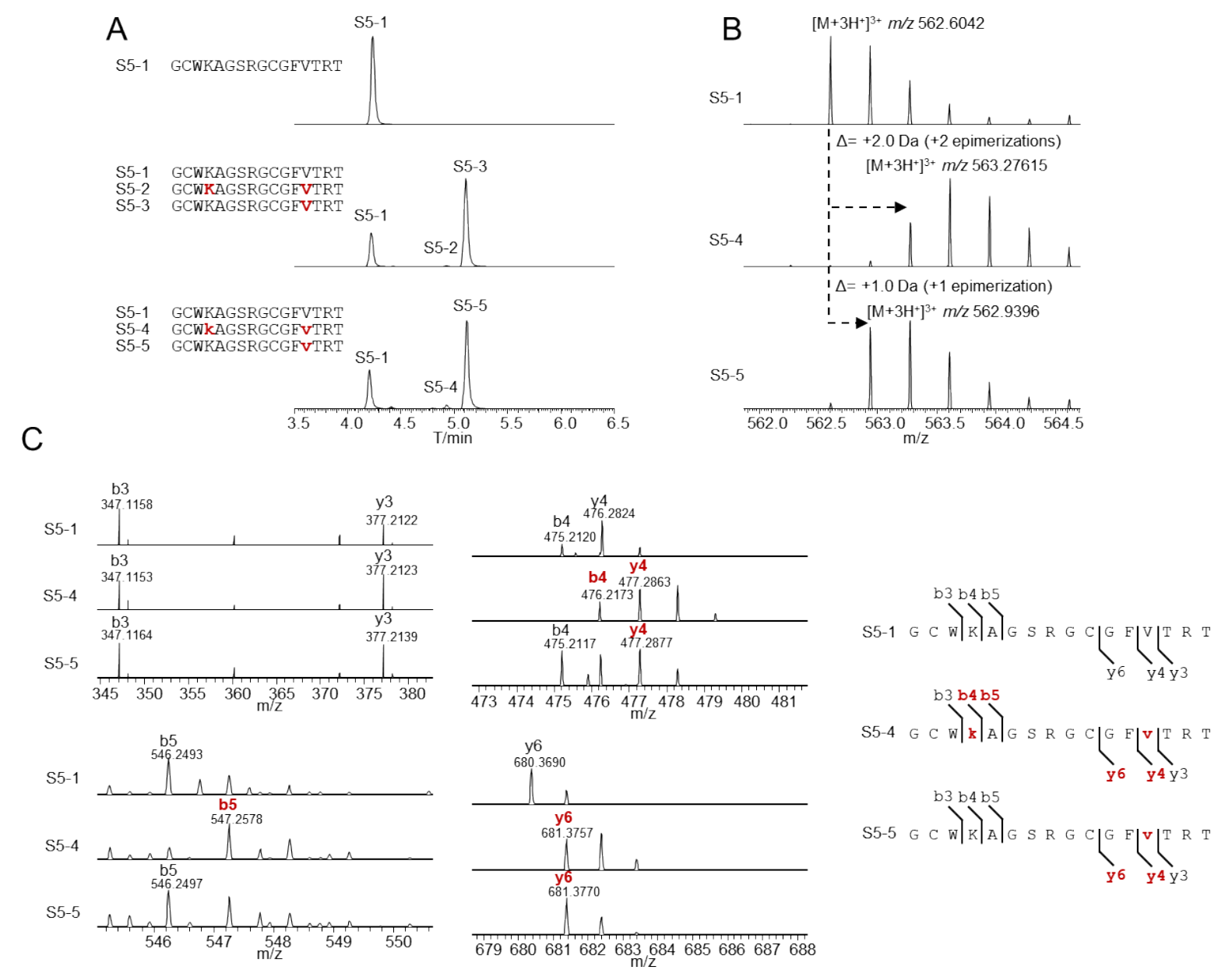

Figure S5. Characterization of selectively labeled products from co-expression of $o s p A-I 4 K+o s p D$ using ODIS. His ${ }_{6}$-OspA variant was cleaved with Factor Xa. (A) EICs of unmodified core peptides (S5-1), core peptides epimerized in TB (S5-2 to S5-3), and epimerized in $\mathrm{D}_{2} \mathrm{O}$-based medium (S5-4 to S5-5). (B) MS for parent ions of S5-1, S5-4 to S5-5 acquired by LC-MS full-scan of ODIS experiment. (C) MS $^{2}$ derived from parent ions of S5-1, S5-4 to S5-5 acquired by LC-MS 2 (PRM) of ODIS experiments. Bold (red) residues indicate epimerized residues. Small letter (red) indicates selective labeling with deuterium at the corresponding epimerized residue. 

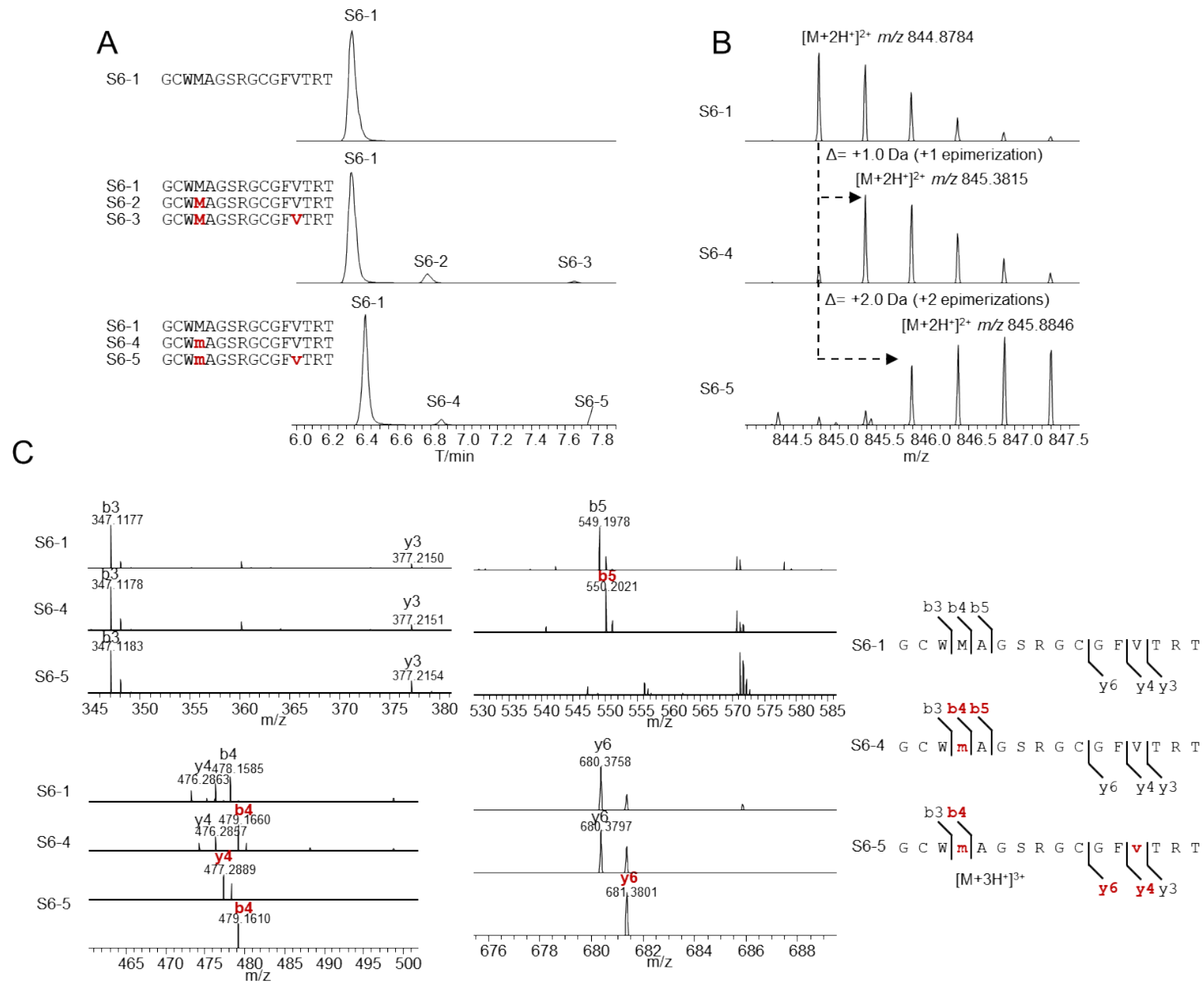

Figure S6. Characterization of selectively labeled products from co-expression of $o s p A-I 4 M+o s p D$ using ODIS. His 6 -OspA variant was cleaved with Factor Xa. (A) EICs of unmodified core peptides (S6-1), core peptides epimerized in TB (S6-2 to S6-3), and epimerized in $\mathrm{D}_{2} \mathrm{O}$-based medium (S6-4 to S6-5). (B) MS for parent ions of S6-1, S6-4 to S6-5 acquired by LC-MS full-scan of ODIS experiment. (C) MS $^{2}$ derived from parent ions of S6-1, S6-4 to S6-5 acquired by LC-MS ${ }^{2}$ (PRM) of ODIS experiment. Bold (red) residues indicate epimerized residues. Small letter (red) indicates selective labeling with deuterium at the corresponding epimerized residue. 


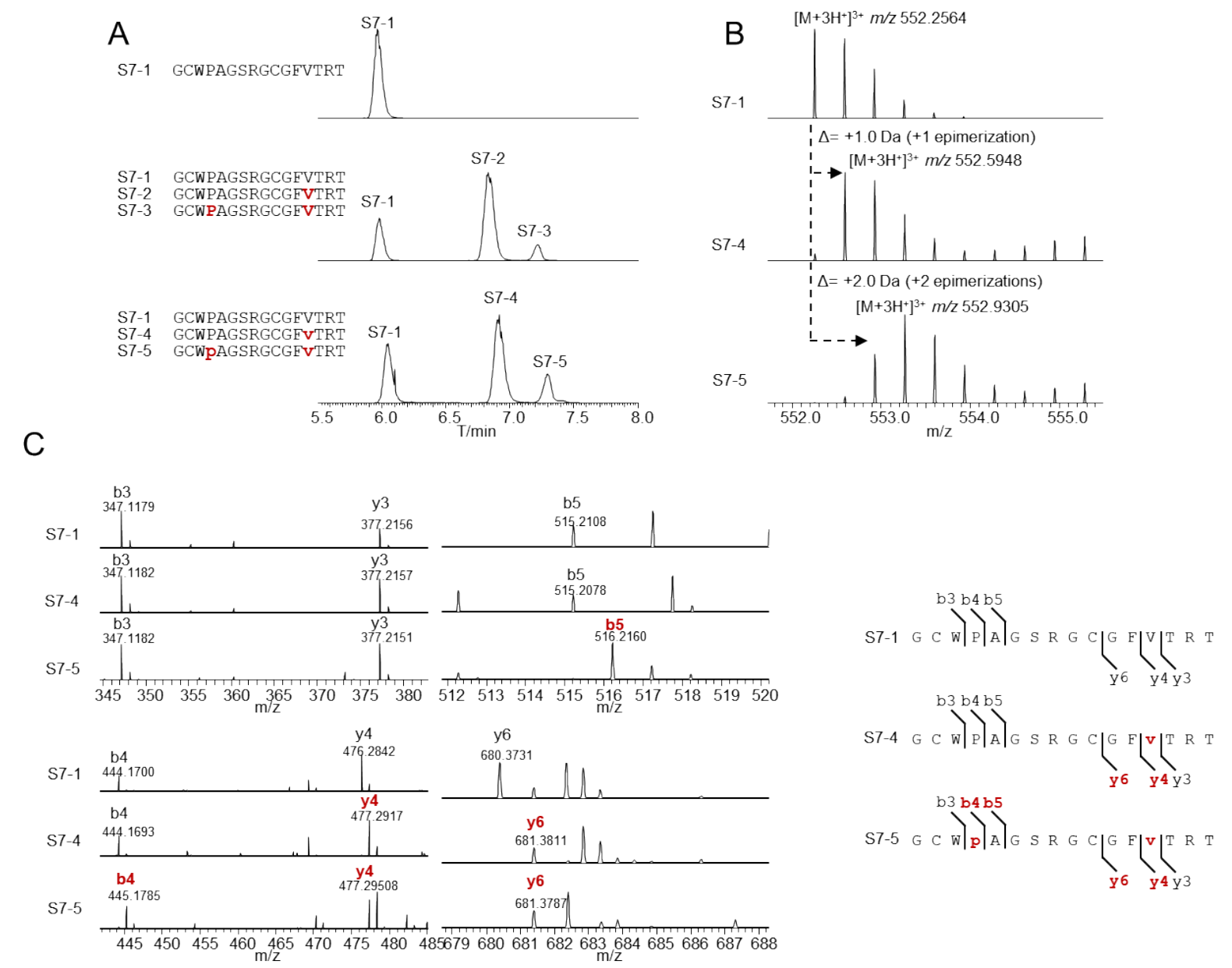

Figure S7. Characterization of selectively labeled products from co-expression of $o s p A-I 4 P+o s p D$ using ODIS. His ${ }_{6}$-OspA variant was cleaved with Factor Xa. (A) EICs of unmodified core peptides (S7-1), core peptides epimerized in TB (S7-2 to S7-3), and epimerized in $\mathrm{D}_{2} \mathrm{O}$-based medium (S7-4 to S7-5). (B) MS for parent ions of S7-1, S7-4 to S7-5 acquired by LC-MS full-scan of ODIS experiment. (C) $\mathrm{MS}^{2}$ derived from parent ions of S7-1, S7-4 to S7-5 acquired by LC-MS ${ }^{2}$ (PRM) of ODIS experiments. Bold (red) residues indicate epimerized residues. Small letter (red) indicates selective labeling with deuterium at the corresponding epimerized residue. 
A

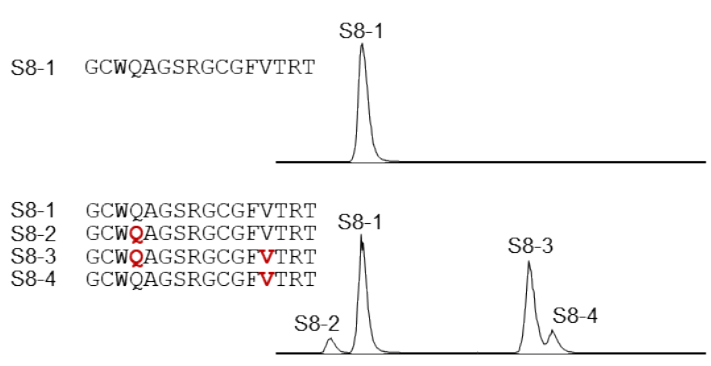

S8-1 GCWQAGSRGCGFVTRT S8-1

S8-5 GCWqAGSRGCGFVTRT

S8-6 GCWqAGSRGCGFVTRT

S8-7 GCWQAGSRGCGEVTRT

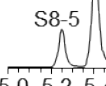

S8-6 S8-7

C
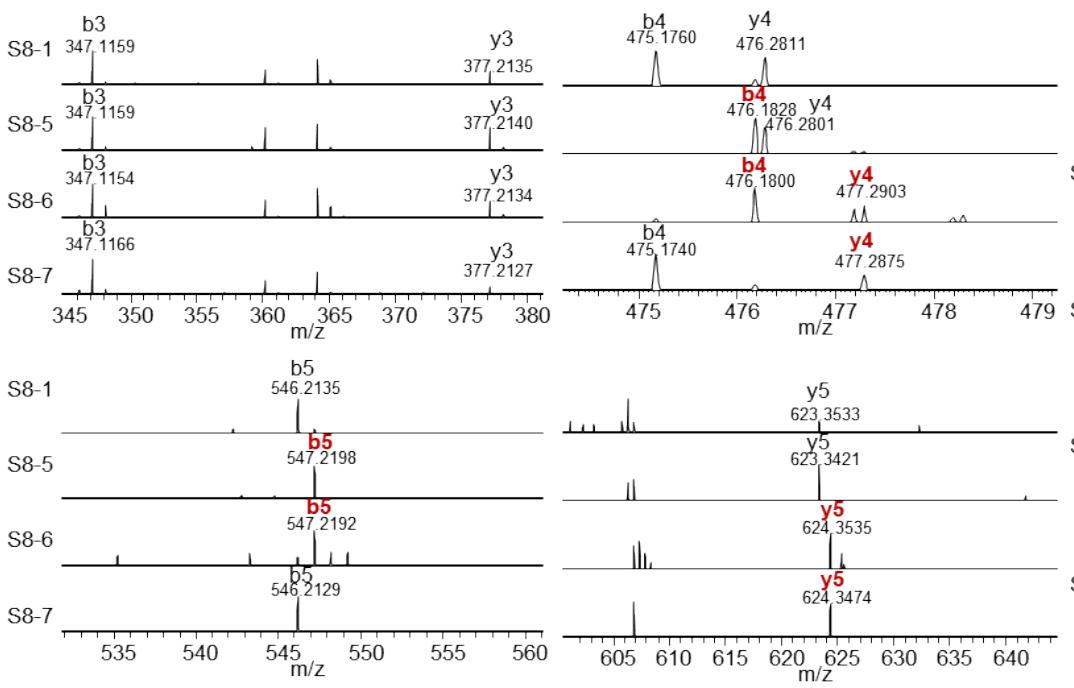

Figure S8. Characterization of selectively labeled products from co-expression of $o s p A-I 4 Q+o s p D$ using ODIS. His ${ }_{6}$-OspA variant was cleaved with Factor Xa. (A) EICs of unmodified core peptides (S8-1), core peptides epimerized in TB (S8-2 to S8-4), and epimerized in $\mathrm{D}_{2} \mathrm{O}$-based medium (S8-5 to S8-7). (B) MS for parent ions of S8-1, S8-5 to S8-7 acquired by LC-MS full-scan of ODIS experiment. (C) $\mathrm{MS}^{2}$ derived from parent ions of S8-1, S8-5 to S8-7 acquired by LC-MS ${ }^{2}$ (PRM) of ODIS experiments. Bold (red) residues indicate epimerized residues. Small letter (red) indicates selective labeling with deuterium at the corresponding epimerized residue.
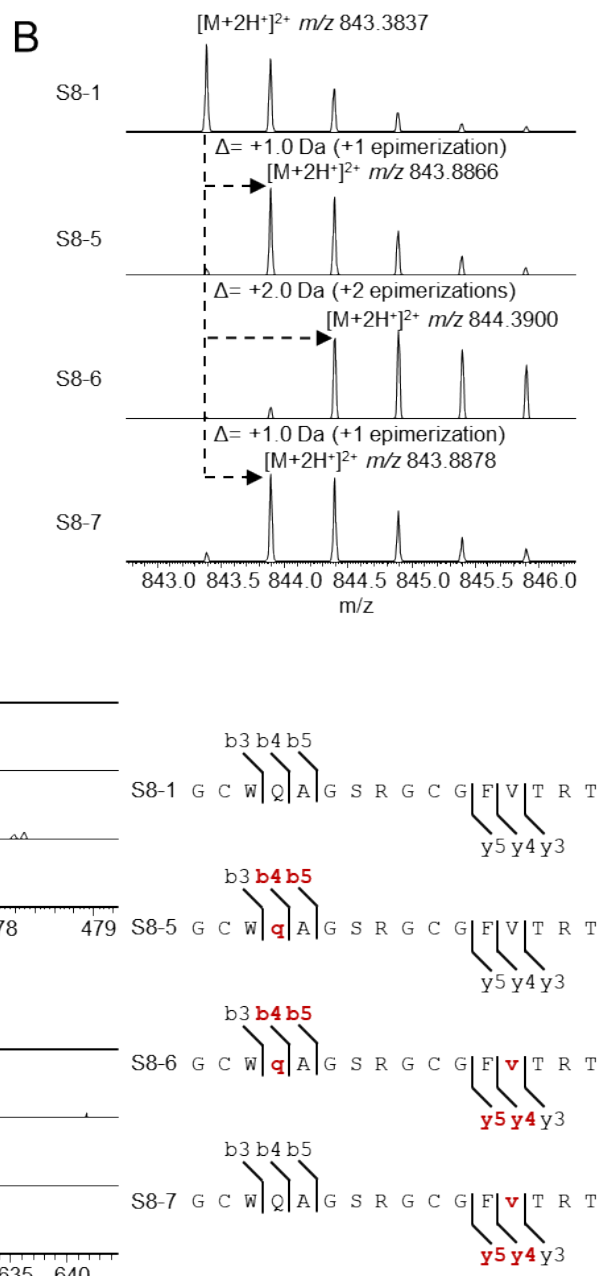


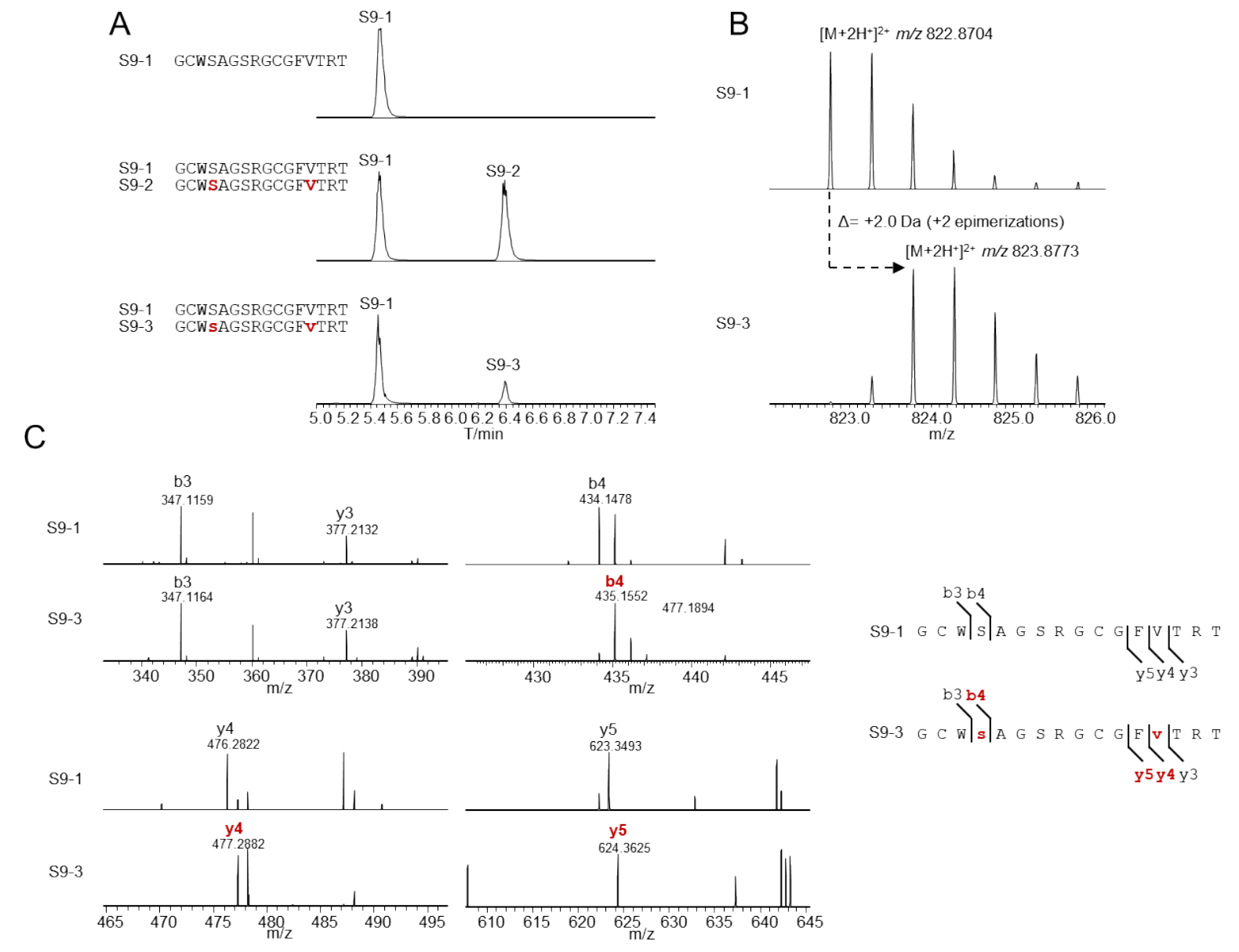

Figure S9. Characterization of selectively labeled products from co-expression of $o s p A-I 4 S+o s p D$ using ODIS. His ${ }_{6}$-OspA variant was cleaved with Factor Xa. (A) EICs of unmodified core peptides (S9-1), core peptides epimerized in TB (S9-2), and epimerized in $\mathrm{D}_{2} \mathrm{O}$-based

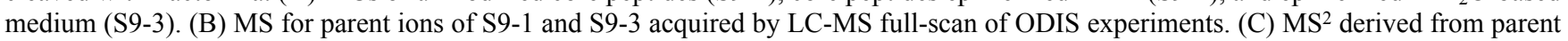
ions of S9-1 and S9-3 acquired by LC-MS² (PRM) of ODIS experiment. Bold (red) residues indicate epimerized residues. Small letter (red) indicates selective labeling with deuterium at the corresponding epimerized residue. 

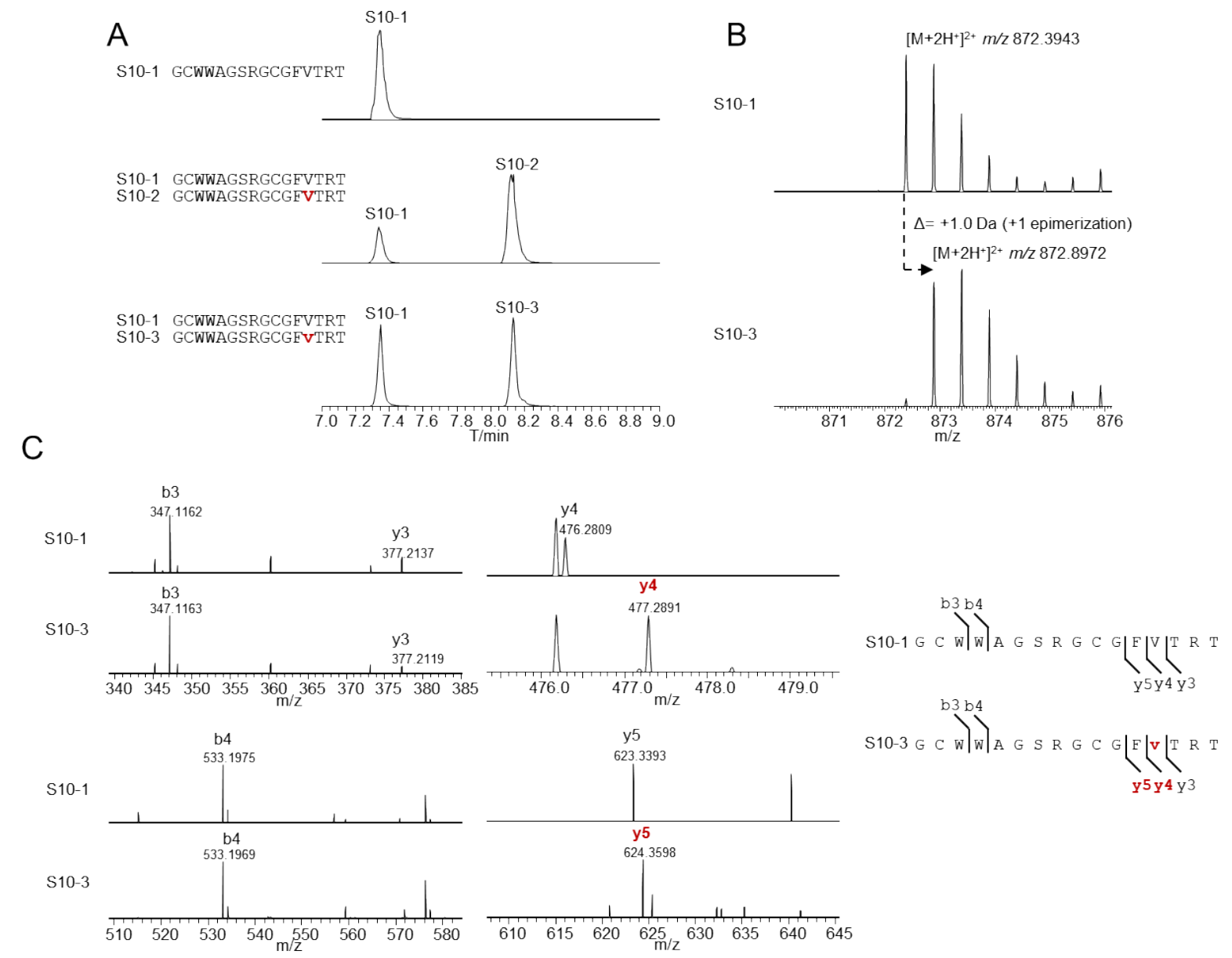

Figure S10. Characterization of selectively labeled products from co-expression of $o s p A-I 4 W+o s p D$ using ODIS. His $6_{6}$-OspA variant was cleaved with Factor Xa. (A) EICs of unmodified core peptides (S10-1), core peptides epimerized in TB (S10-2), and epimerized in $\mathrm{D}_{2} \mathrm{O}-$ based medium (S10-3). (B) MS for parent ions of S10-1 and S10-3 acquired by LC-MS full-scan of ODIS experiment. (C) MS ${ }^{2}$ derived from parent ions of S10-1 and S10-3 acquired by LC-MS 2 (PRM) of ODIS experiments. Bold (red) residues indicate epimerized residues. Small letter (red) indicates selective labeling with deuterium at the corresponding epimerized residue. 


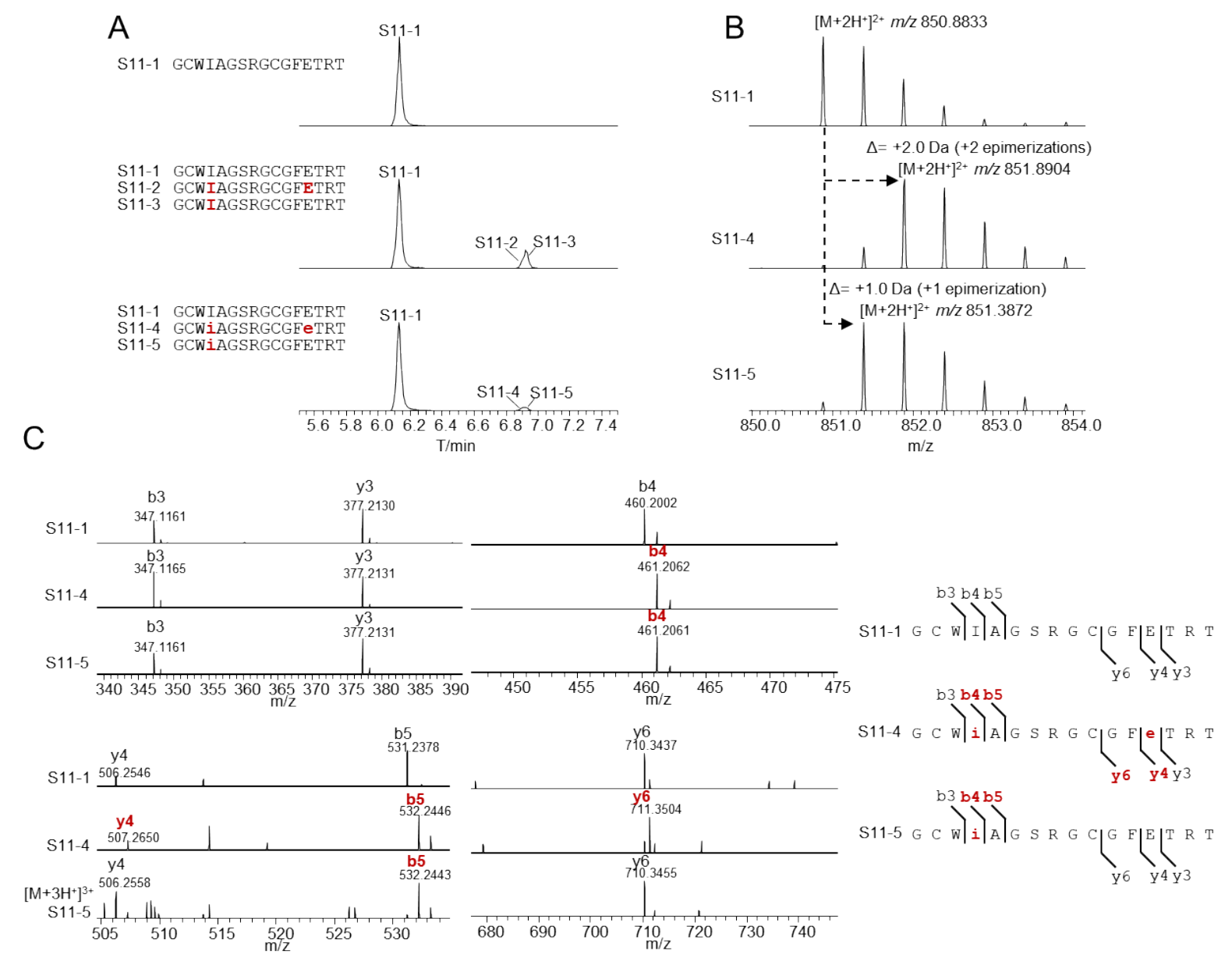

Figure S11. Characterization of selectively labeled products from co-expression of $o s p A-V 13 E+o s p D$ using ODIS. His ${ }_{6}$-OspA variant was cleaved with Factor Xa. (A) EICs of unmodified core peptides (S11-1), core peptides epimerized in TB (S11-2 to S11-3), and epimerized in $\mathrm{D}_{2} \mathrm{O}$-based medium (S11-4 to S11-5). (B) MS for parent ions of S11-1, S11-4 to S11-5 acquired by LC-MS full-scan of ODIS experiment. (C) $\mathrm{MS}^{2}$ derived from parent ions of S11-1, S11-4 to S11-5 acquired by LC-MS² (PRM) of ODIS experiments. Bold (red) residues indicate epimerized residues. Small letter (red) indicates selective labeling with deuterium at the corresponding epimerized residue. 


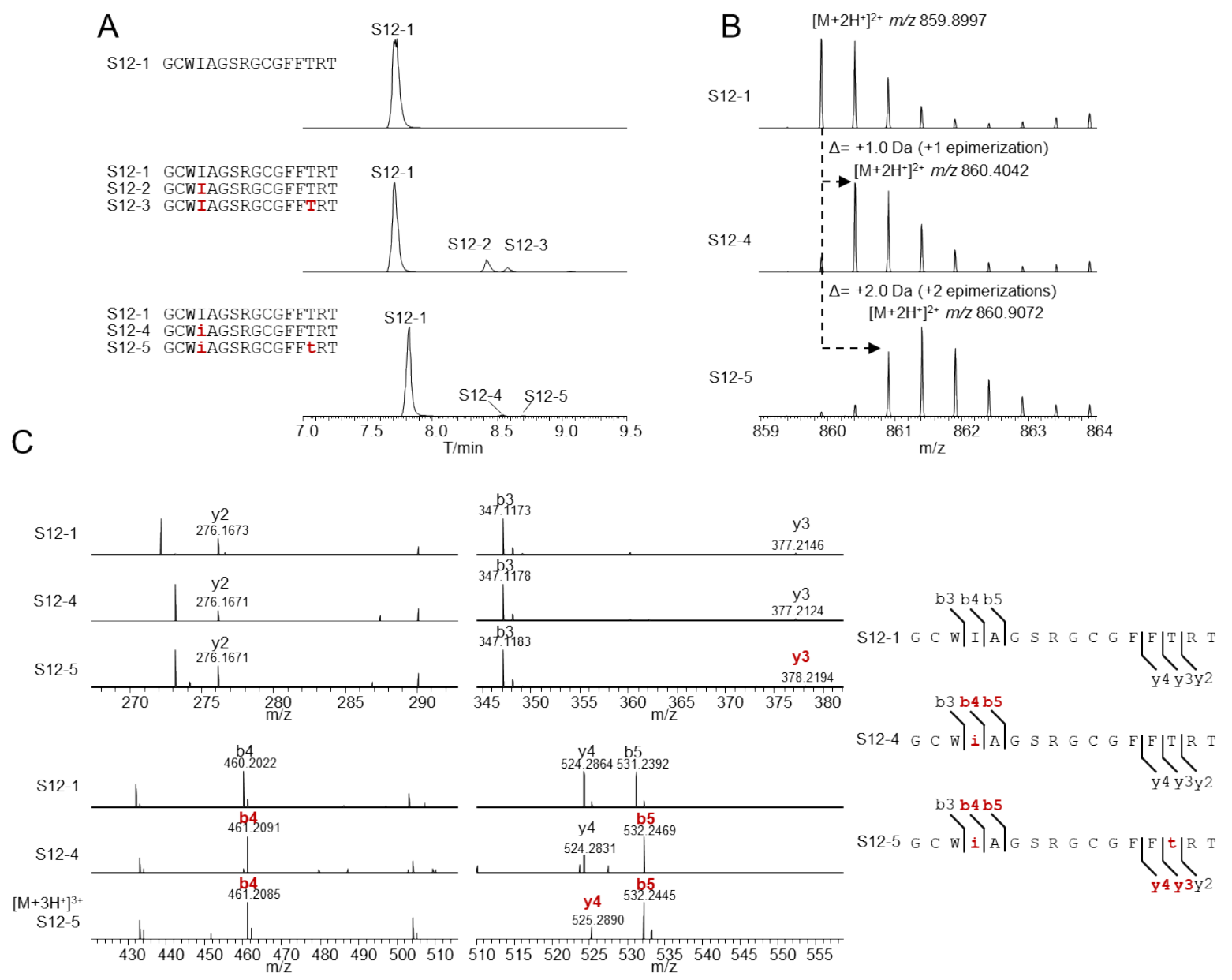

Figure S12. Characterization of selectively labeled products from co-expression of $o s p A-V 13 F+o s p D$ using ODIS. His 6 -OspA variant was cleaved with Factor Xa. (A) EICs of unmodified core peptides (S12-1), core peptides epimerized in TB (S12-2 to S12-3), and epimerized in $\mathrm{D}_{2} \mathrm{O}$-based medium (S12-4 to S12-5). (B) MS for parent ions of S12-1, S12-4 to S12-5 acquired by LC-MS full-scan of ODIS experiment. (C) $\mathrm{MS}^{2}$ derived from parent ions of S12-1, S12-4 to S12-5 acquired by LC-MS2 (PRM) of ODIS experiments. Bold (red) residues indicate epimerized residues. Small letter (red) indicates selective labeling with deuterium at the corresponding epimerized residue. 


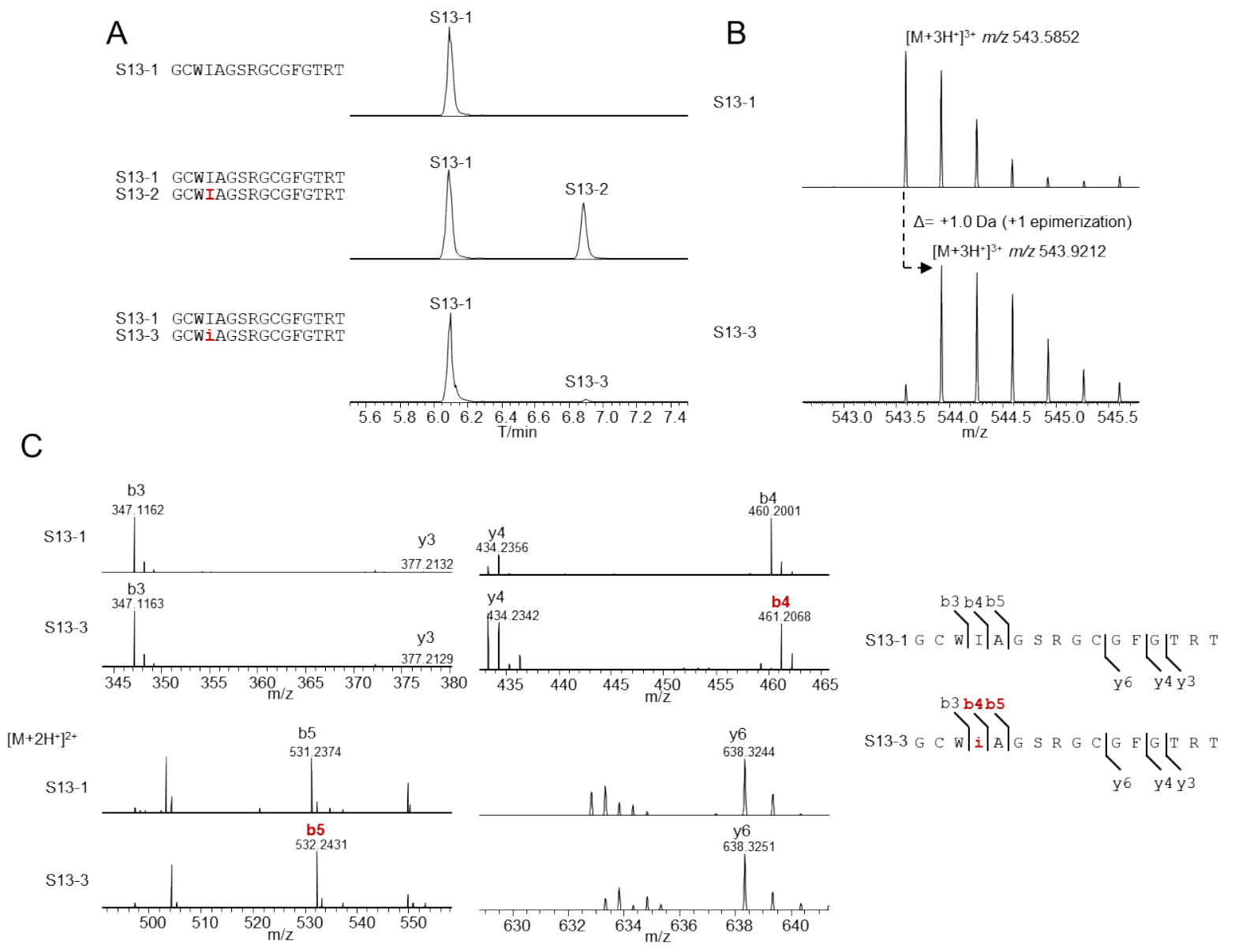

Figure S13. Characterization of selectively labeled products from co-expression of $o s p A-V 13 G+o s p D$ using ODIS. His 6 -OspA variant was cleaved with Factor Xa. (A) EICs of unmodified core peptides (S13-1), core peptides epimerized in TB (S13-2), and epimerized in $\mathrm{D}_{2} \mathrm{O}-$ based medium (S13-3). (B) MS for parent ions of S13-1 and S13-3 acquired by LC-MS full-scan of ODIS experiment. (C) MS ${ }^{2}$ derived from parent ions of S13-1 and S13-3 acquired by LC-MS 2 (PRM) of ODIS experiments. Bold (red) residues indicate epimerized residues. Small letter (red) indicates selective labeling with deuterium at the corresponding epimerized residue. 

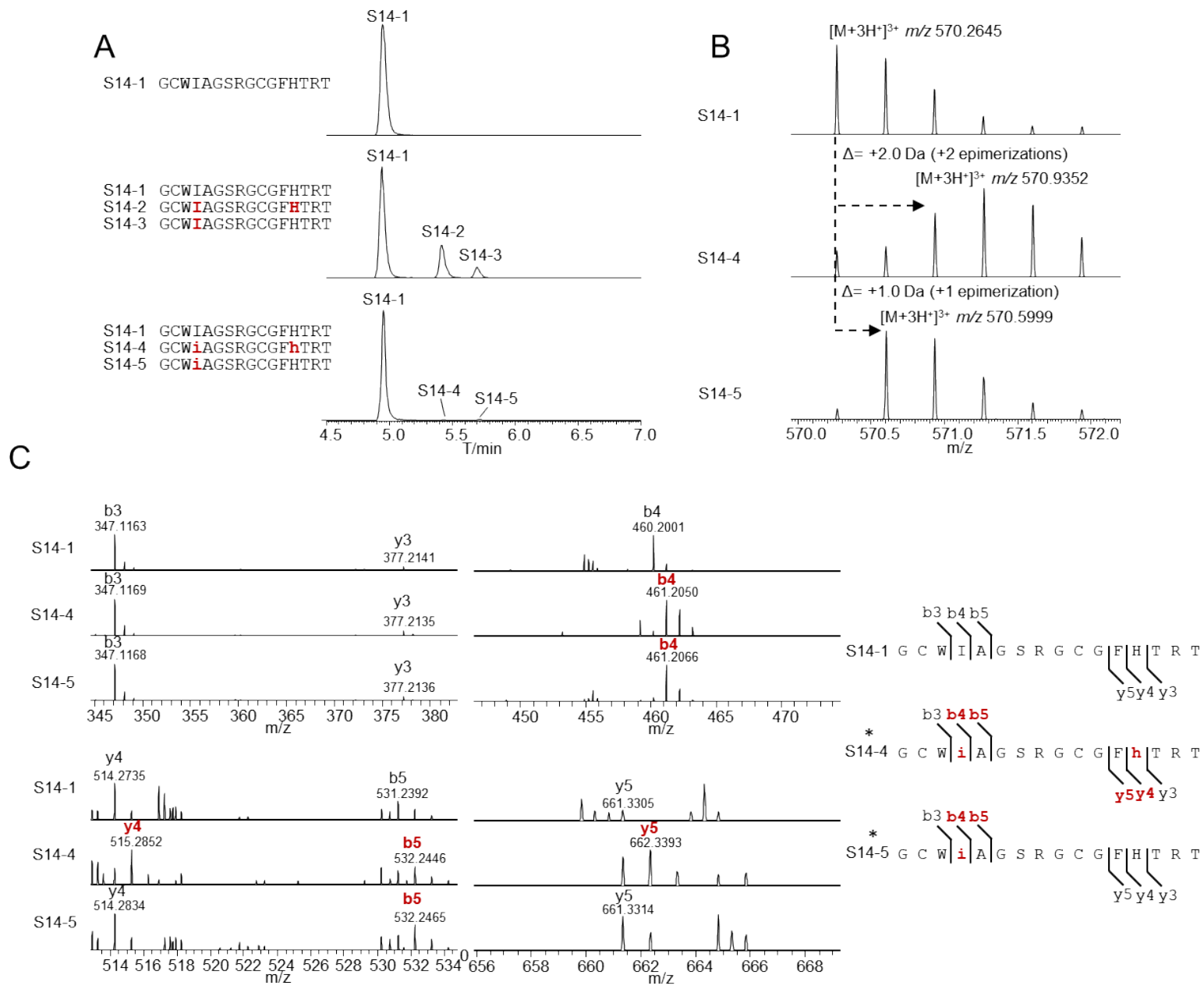

Figure S14. Characterization of selectively labeled products from co-expression of $o s p A-V 13 H+o s p D$ using ODIS. His ${ }_{6}$-OspA variant was cleaved with Factor Xa. (A) EICs of unmodified core peptides (S14-1), core peptides epimerized in TB (S14-2 to S14-3), and epimerized in $\mathrm{D}_{2} \mathrm{O}$-based medium (S14-4 to S14-5). (B) MS for parent ions of S14-1, S14-4 to S14-5 acquired by LC-MS full-scan of ODIS experiment. (C) MS 2 derived from parent ions of S14-1, S14-4 to S14-5 acquired by LC-MS² (PRM) of ODIS experiments. Bold (red) residues indicate epimerized residues. Small letter (red) indicates selective labeling with deuterium at the corresponding epimerized residue. Repeated measurements for improved $\mathrm{MS}^{2}$ data are indicated with asterisks. 


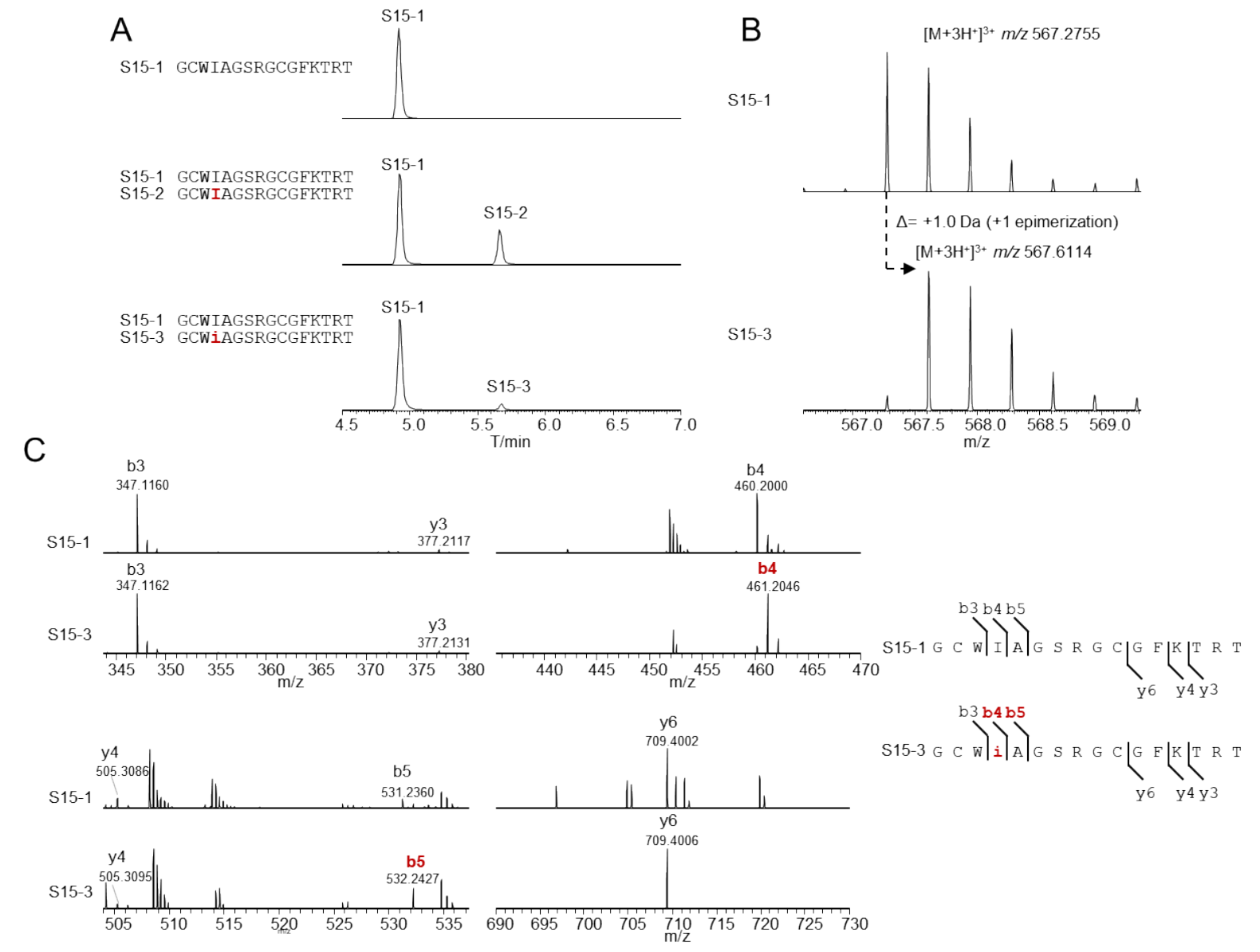

Figure S15. Characterization of selectively labeled products from co-expression of osp $A-V 13 K+o s p D$ using ODIS. His ${ }_{6}$-OspA variant was cleaved with Factor Xa. (A) EICs of unmodified core peptides (S15-1), core peptides epimerized in TB (S15-2), and epimerized in $\mathrm{D}_{2} \mathrm{O}-$ based medium (S15-3). (B) MS for parent ions of S15-1 and S15-3 acquired by LC-MS full-scan of ODIS experiment. (C) MS ${ }^{2}$ derived from parent ions of S15-1 and S15-3 acquired by LC-MS ${ }^{2}$ (PRM) of ODIS experiments. Bold (red) residues indicate epimerized residues. Small letter (red) indicates selective labeling with deuterium at the corresponding epimerized residue. 


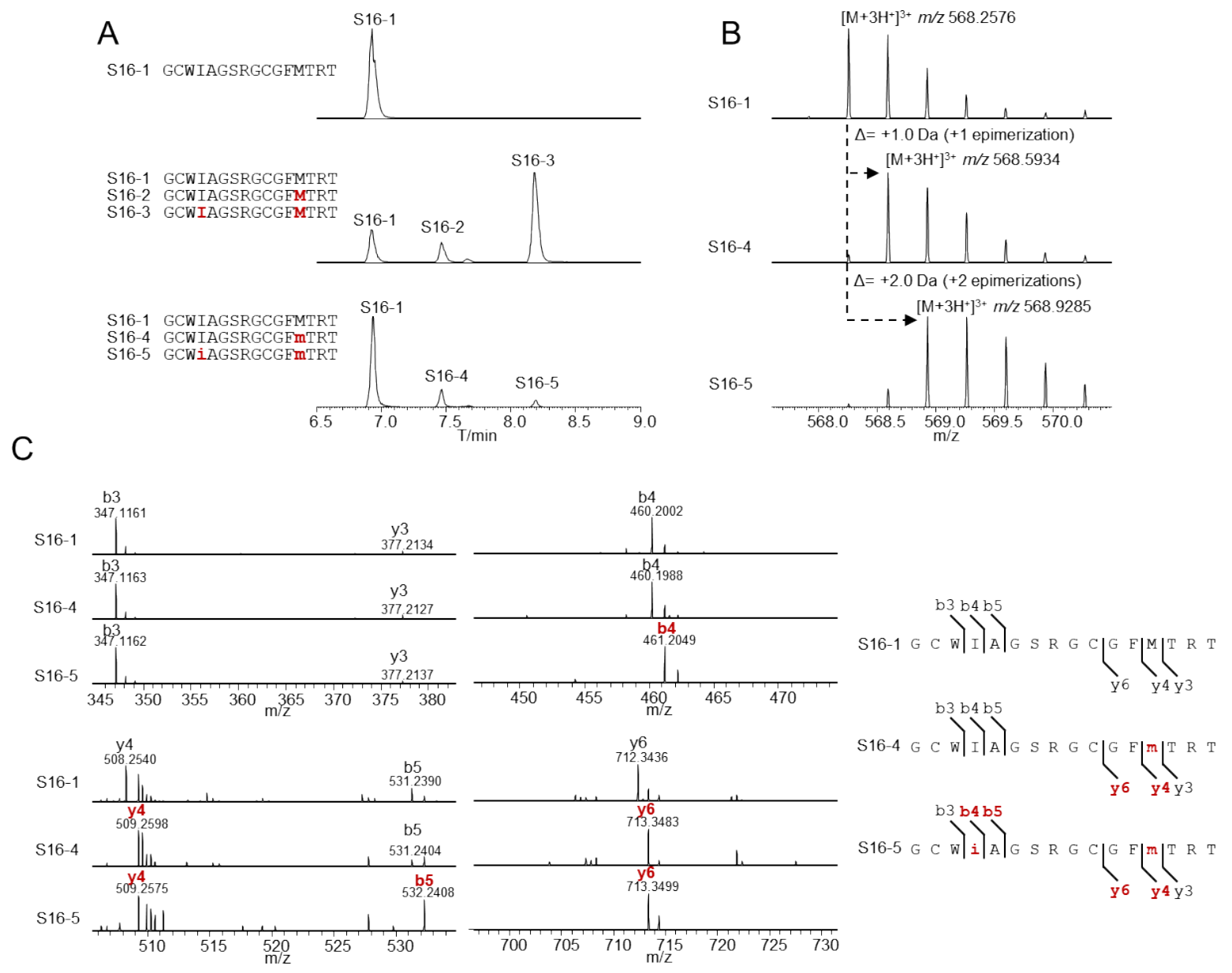

Figure S16. Characterization of selectively labeled products from co-expression of $o s p A-V 13 M+o s p D$ using ODIS. His 6 -OspA variant was cleaved with Factor Xa. (A) EICs of unmodified core peptides (S16-1), core peptides epimerized in TB (S16-2 to S16-3), and epimerized in $\mathrm{D}_{2} \mathrm{O}$-based medium (S16-4 to S16-5). (B) MS for parent ions of S16-1, S16-4 to S16-5 acquired by LC-MS full-scan of ODIS experiment. (C) $\mathrm{MS}^{2}$ derived from parent ions of S16-1, S16-4 to S16-5 acquired by LC-MS² (PRM) of ODIS experiments. Bold (red) residues indicate epimerized residues. Small letter (red) indicates selective labeling with deuterium at the corresponding epimerized residue. 


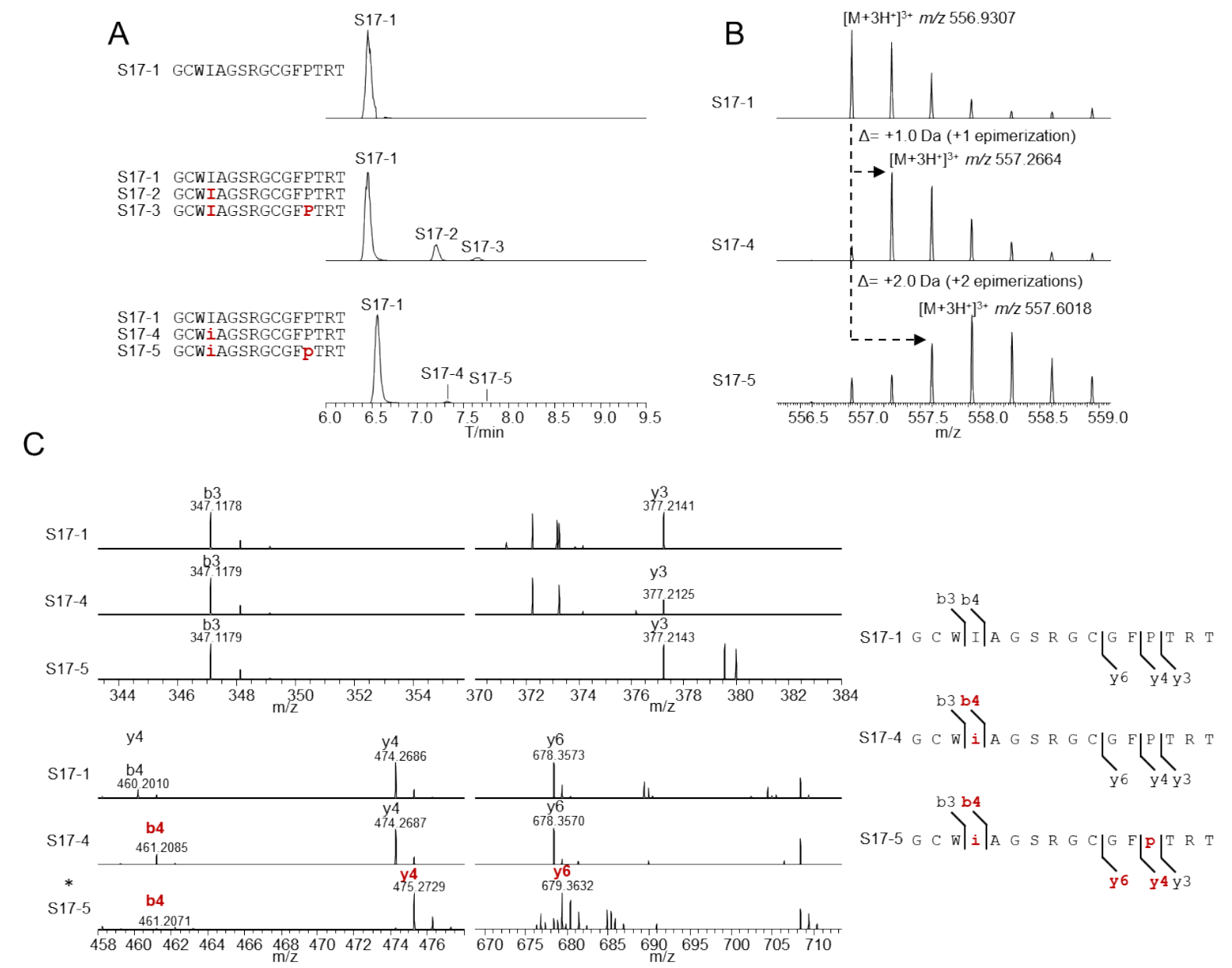

Figure S17. Characterization of selectively labeled products from co-expression of $o s p A-V 13 P+o s p D$ using ODIS. His ${ }_{6}$-OspA variant was cleaved with Factor Xa. (A) EICs of unmodified core peptides (S17-1), core peptides epimerized in TB (S17-2 to S17-3), and epimerized in $\mathrm{D}_{2} \mathrm{O}$-based medium (S17-4 to S17-5). (B) MS for parent ions of S17-1, S17-4 to S17-5 acquired by LC-MS full-scan of ODIS experiment. (C) $\mathrm{MS}^{2}$ derived from parent ions of S17-1, S17-4 to S17-5 acquired by LC-MS² (PRM) of ODIS experiments. Bold (red) residues indicate epimerized residues. Small letter (red) indicates selective labeling with deuterium at the corresponding epimerized residue. Repeated measurements for improved $\mathrm{MS}^{2}$ data are indicated with an asterisk. 


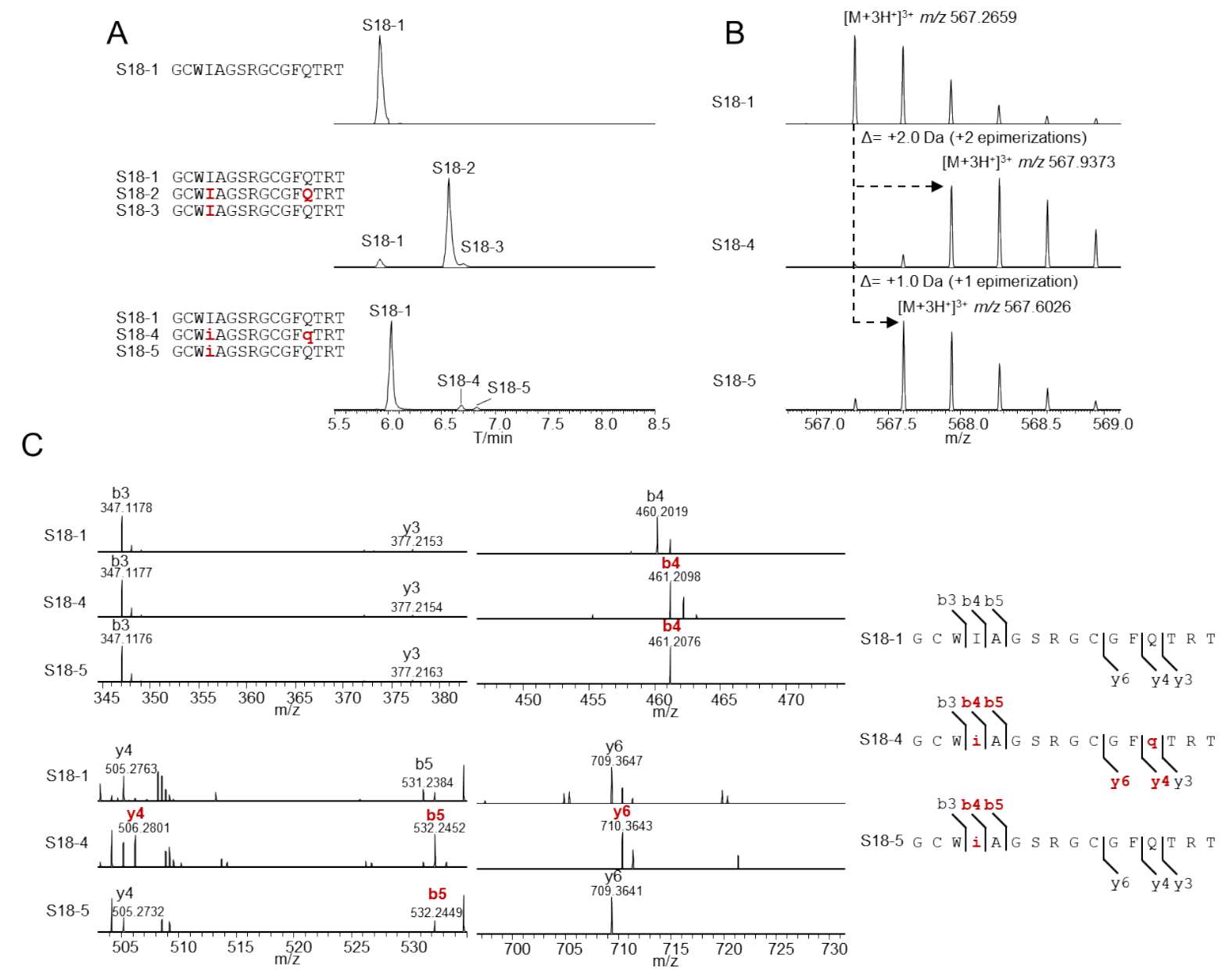

Figure S18. Characterization of selectively labeled products from co-expression of $o s p A-V 13 Q+o s p D$ using ODIS. His 6 -OspA variant was cleaved with Factor Xa. (A) EICs of unmodified core peptides (S18-1), core peptides epimerized in TB (S18-2 to S18-3), and epimerized in $\mathrm{D}_{2} \mathrm{O}$-based medium (S18-4 to S18-5). (B) MS for parent ions of S18-1, S18-4 to S18-5 acquired by LC-MS full-scan of ODIS experiment. (C) MS 2 derived from parent ions of S18-1, S18-4 to S18-5 acquired by LC-MS² (PRM) of ODIS experiments. Bold (red) residues indicate epimerized residues. Small letter (red) indicates selective labeling with deuterium at the corresponding epimerized residue. 


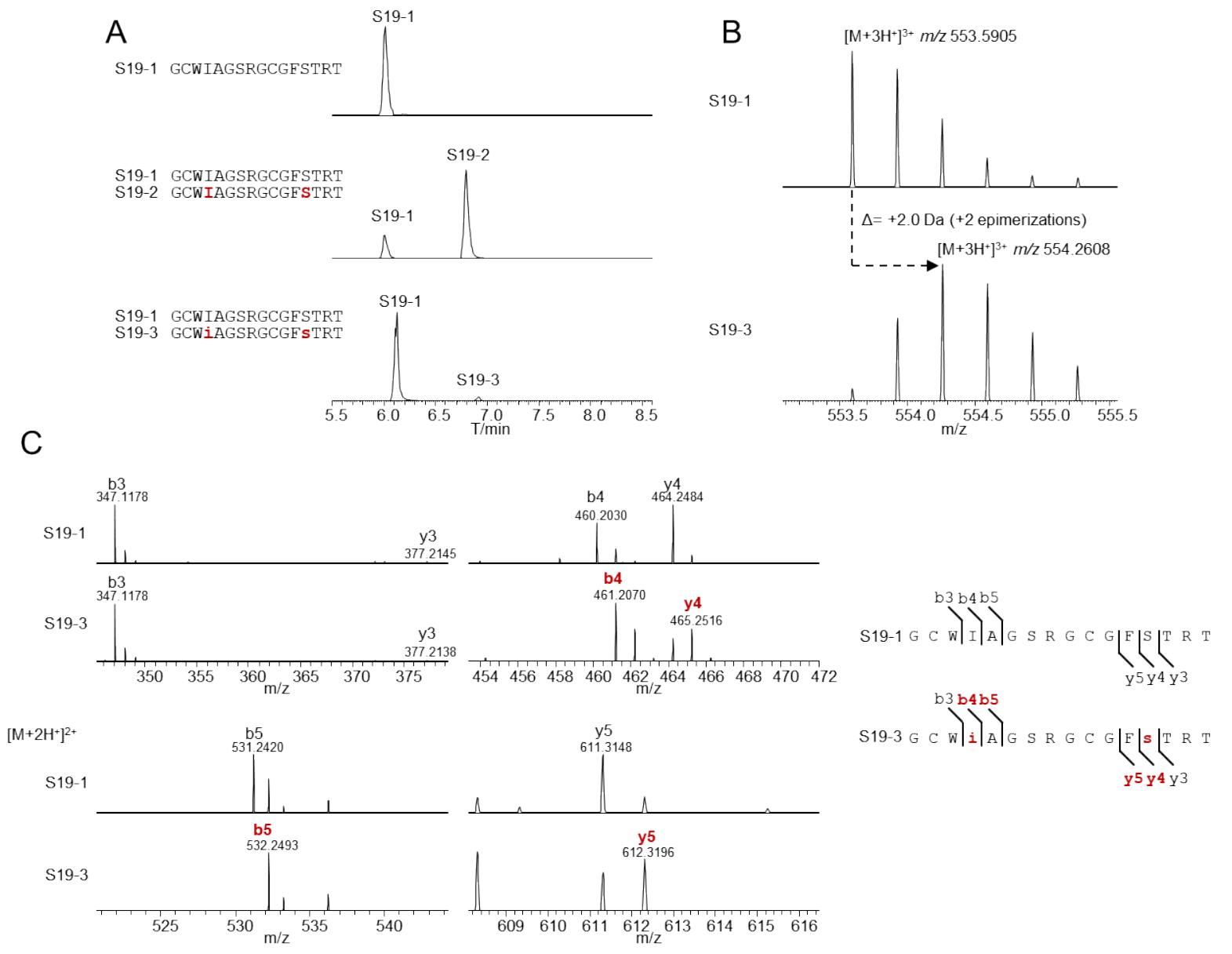

Figure S19. Characterization of selectively labeled products from co-expression of $o s p A-V 13 S+o s p D$ using ODIS. His ${ }_{6}$-OspA variant was cleaved with Factor Xa. (A) EICs of unmodified core peptides (S19-1), core peptides epimerized in TB (S19-2), and epimerized in $\mathrm{D}_{2} \mathrm{O}-$ based medium (S19-3). (B) MS for parent ions of S19-1 and S19-3 acquired by LC-MS full-scan of ODIS experiment. (C) MS ${ }^{2}$ derived from parent ions of S19-1 and S19-3 acquired by LC-MS 2 (PRM) of ODIS experiments. Bold (red) residues indicate epimerized residues. Small letter (red) indicates selective labeling with deuterium at the corresponding epimerized residue. 
A

S20-1 GCWIAGSRGCGFWTRT

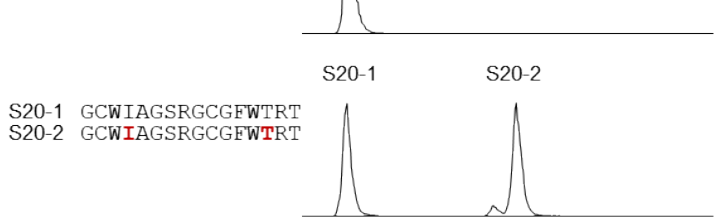

S20-1 GCWIAGSRGCGFWTR'
S20-3 GCWIAGSRGCGFWtR'

S20-1

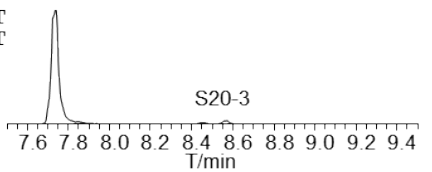

C
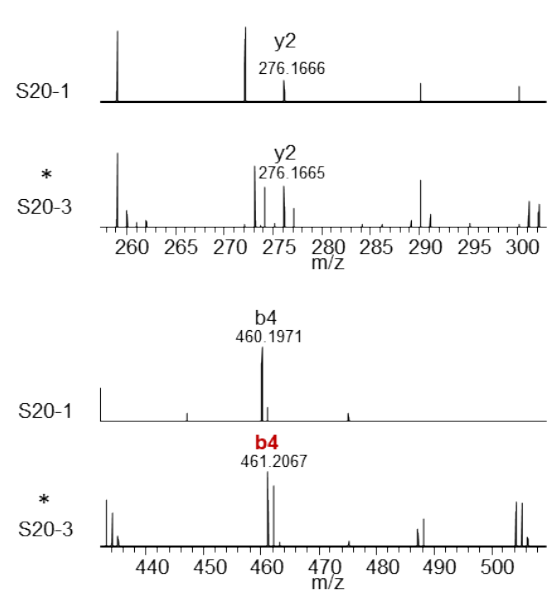

B

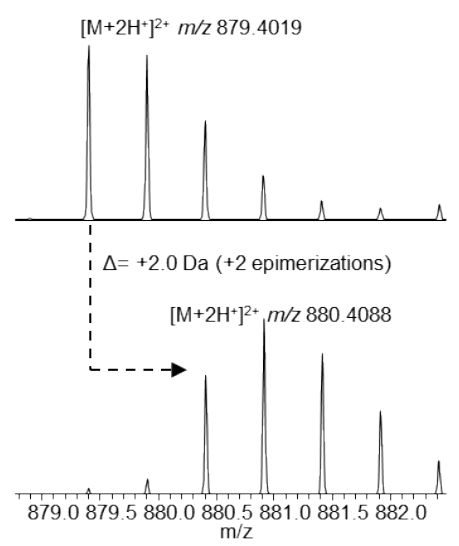

Figure S20. Characterization of selectively labeled products from co-expression of $\operatorname{ssp} A-V 13 W+o s p D$ using ODIS. His 6 -OspA variant was cleaved with Factor Xa. (A) EICs of unmodified core peptides (S20-1), core peptides epimerized in TB (S20-2), and epimerized in $\mathrm{D}_{2} \mathrm{O}-$ based medium (S20-3). (B) MS for parent ions of S20-1 and S20-3 acquired by LC-MS full-scan of ODIS experiment. (C) MS² derived from parent ions of S20-1 and S20-3 acquired by LC-MS 2 (PRM) of ODIS experiments. Bold (red) residues indicate epimerized residues. Small letter (red) indicates selective labeling with deuterium at the corresponding epimerized residue. Repeated measurements for improved $\mathrm{MS}^{2}$ data are indicated with asterisks. 


\section{A}

S21-1 GCAIAGSRGCGFVTRT

S21-1 GCAIAGSRGCGFVTRT

S21-2 GCAIAGSRGCGEVTRT

S21-3 GCAIAGSRGCGFVTRT

S21-4 GCAIAGSRGCGEVTRT

S21-1 GCAIAGSRGCGFVTRT

S21-5 GCAIAGSRGCGFVTR

S21-6 GCAiAGSRGCGFvTR

S21-7 GCAiAGSRGCGFVTRT

C

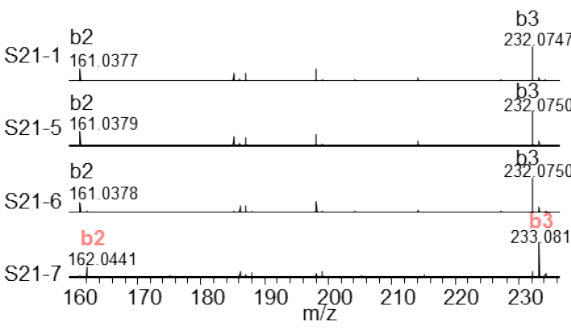

$\begin{array}{lllll}4 & 4.5 & 5.0 & 5.5 & 6.0 \\ \mathrm{~T} / \mathrm{min}\end{array}$
B

$\mathrm{S} 21-1$

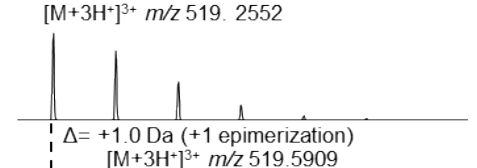

S21-5

S21-6

$\mathrm{S} 21-7$

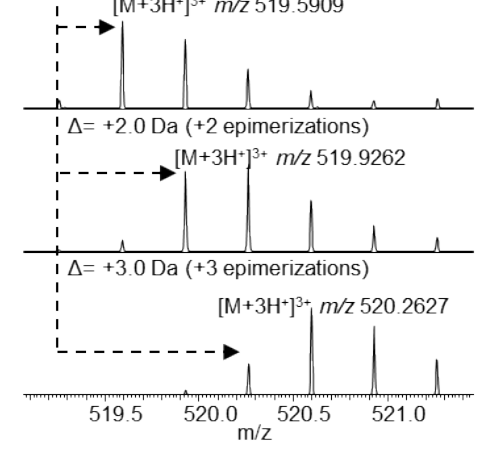

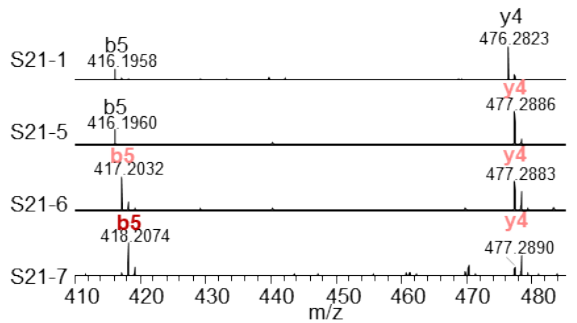
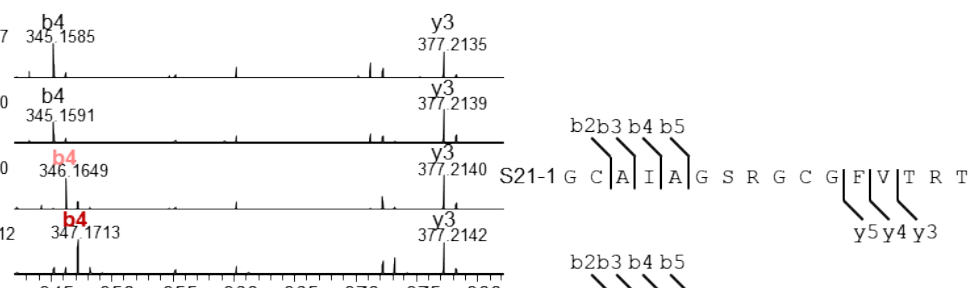

S21-5 G C
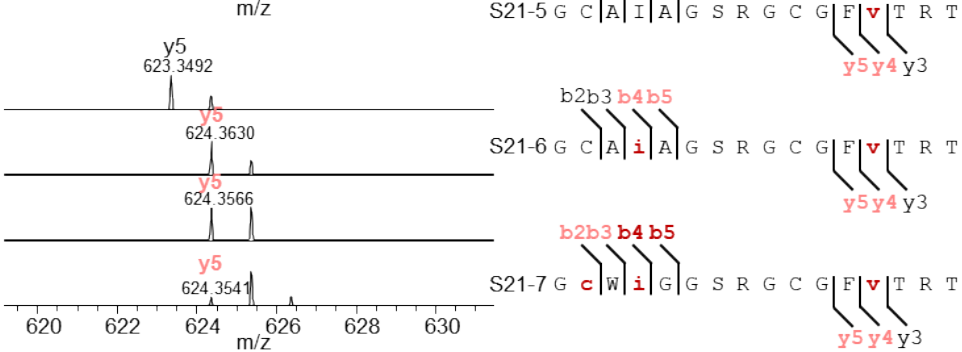

Figure S21. Characterization of selectively labeled products from co-expression of $o s p A-W 3 A+o s p D$ using ODIS. His ${ }_{6}$-OspA variant was cleaved with Factor Xa. (A) EICs of unmodified core peptides (S21-1), core peptides epimerized in TB (S21-2 to S21-4), and epimerized in $\mathrm{D}_{2} \mathrm{O}$-based medium (S21-5 to S21-7). (B) MS for parent ions of S21-1, S21-5 to S21-7 acquired by LC-MS full-scan of ODIS experiment. (C) $\mathrm{MS}^{2}$ derived from parent ions of S21-1, S21-5 to S21-7 acquired by LC-MS² (PRM) of ODIS experiments. Bold (red) residues indicate epimerized residues. Small letter (red) indicates selective labeling with deuterium at the corresponding epimerized residue. Rose-colored b/y ions have mass shift of +1.0 Da, red b/y ions of +2.0 Da. 


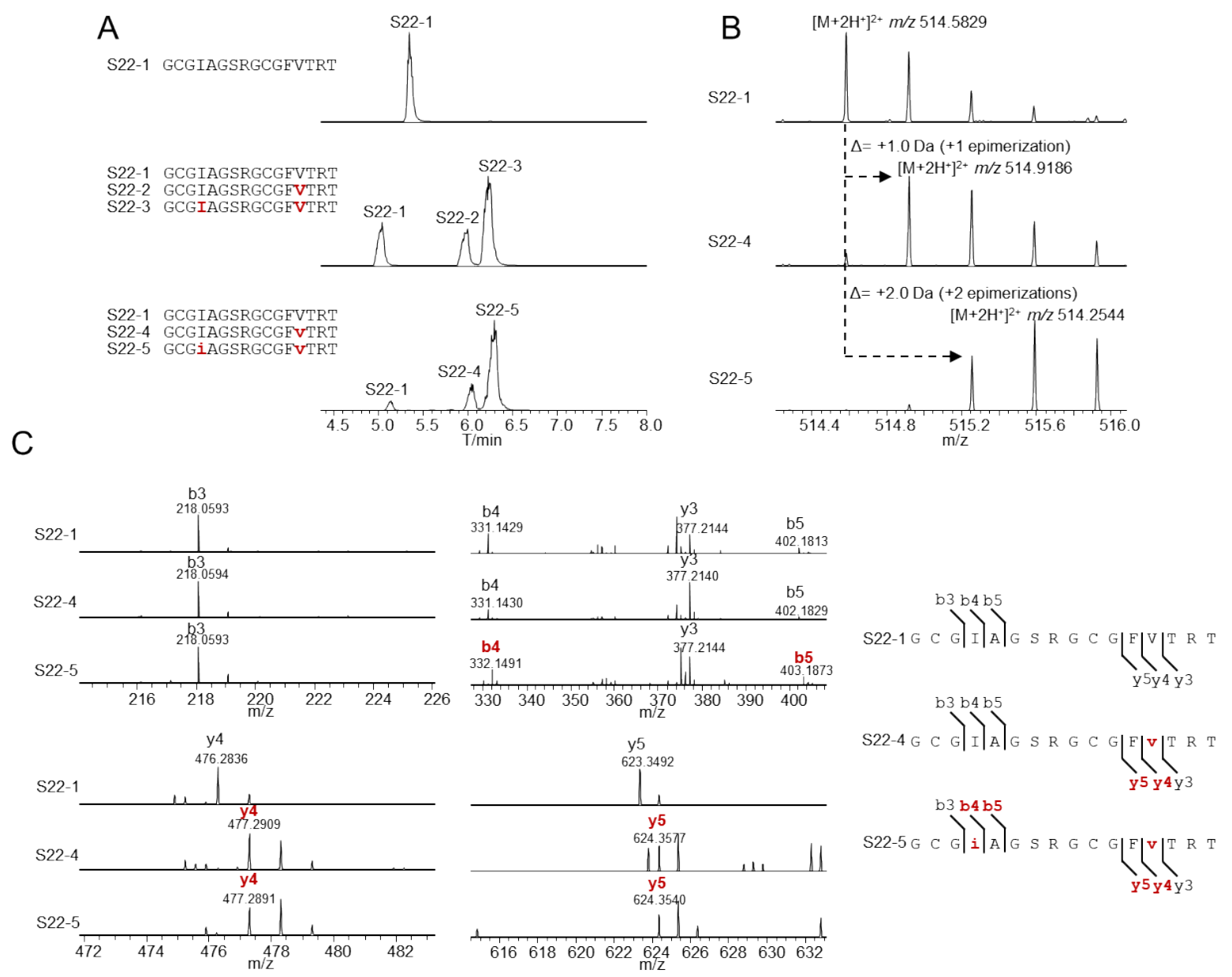

Figure S22. Characterization of selectively labeled products from co-expression of $o s p A-W 3 G+o s p D$ using ODIS. His $6_{6}$-OspA variant was cleaved with Factor Xa. (A) EICs of unmodified core peptides (S22-1), core peptides epimerized in TB (S22-2 to S22-3), and epimerized in $\mathrm{D}_{2} \mathrm{O}$-based medium (S22-4 to S22-5). (B) MS for parent ions of S22-1, S22-4 to S22-5 acquired by LC-MS full-scan of ODIS experiment. (C) $\mathrm{MS}^{2}$ derived from parent ions of S22-1, S22-4 to S22-5 acquired by LC-MS² (PRM) of ODIS experiments. Bold (red) residues indicate epimerized residues. Small letter (red) indicates selective labeling with deuterium at the corresponding epimerized residue. 


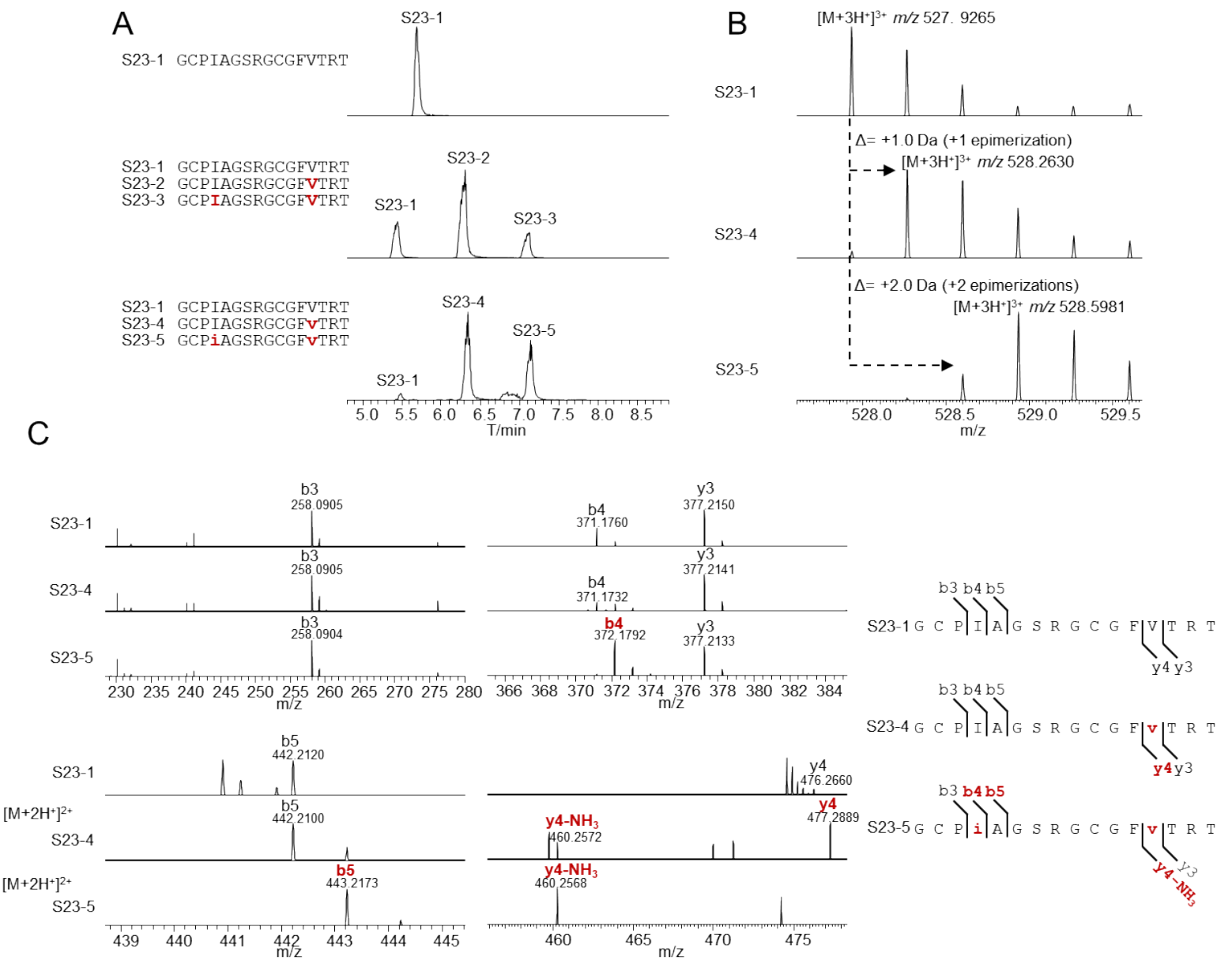

Figure S23. Characterization of selectively labeled products from co-expression of $o s p A-W 3 P+o s p D$ using ODIS. His ${ }_{6}$-OspA variant was cleaved with Factor Xa. (A) EICs of unmodified core peptides (S23-1), core peptides epimerized in TB (S23-2 to S23-3), and epimerized in $\mathrm{D}_{2} \mathrm{O}$-based medium (S23-4 to S23-5). (B) MS for parent ions of S23-1, S23-4 to S23-5 acquired by LC-MS full-scan of ODIS experiment. (C) MS 2 derived from parent ions of S23-1, S23-4 to S23-5 acquired by LC-MS 2 (PRM) of ODIS experiments. Bold (red) residues indicate epimerized residues. Small letter (red) indicates selective labeling with deuterium at the corresponding epimerized residue. 
A S24-1 GCWIGGSRGCGFVTRT S24-1 GCWIGGSRCGEVRT

S24-1 GCWIGGSRGCGFVTRT S24-2 GCWIGGSRGCGFVTRT S24-3 GCWIGGSRGCGFVTRT

S24-1 GCWIGGSRGCGFVTRT S24-5 GCWiGGSRGCGFVTRT $\begin{array}{ll}\text { S24-6 } & \text { GCWIGGSRGCGEVTRT } \\ \text { S24-7 } & \text { GCWiGGSRGCGFVTRT }\end{array}$

\section{C}

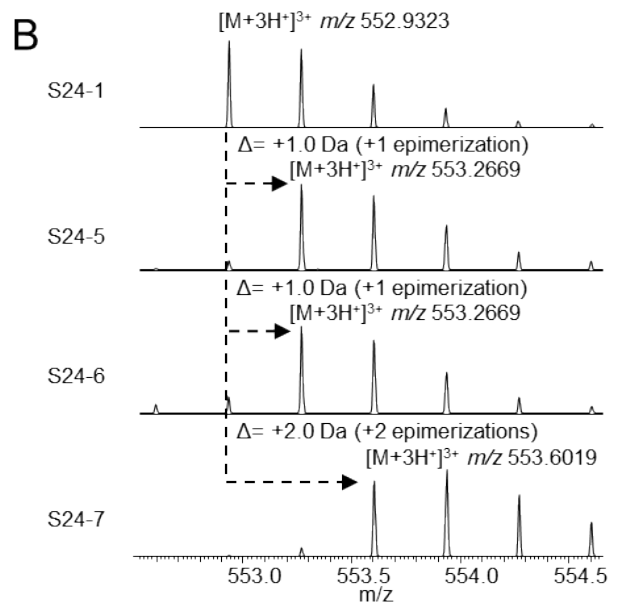

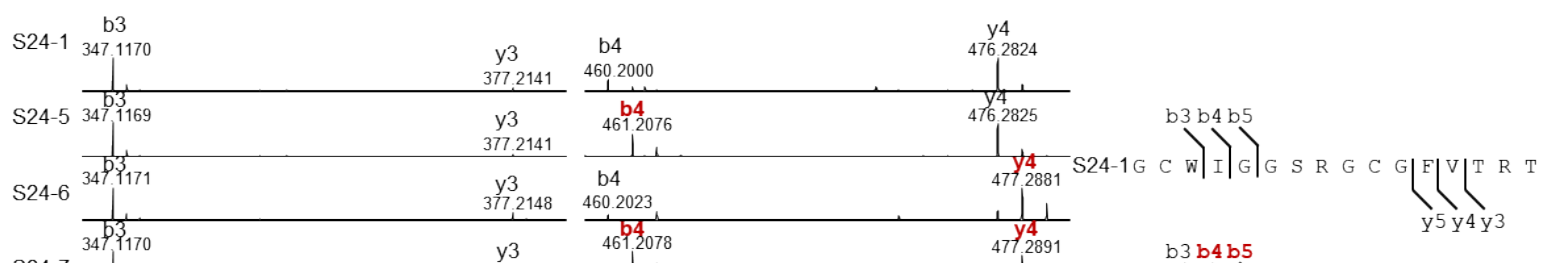

S24-7
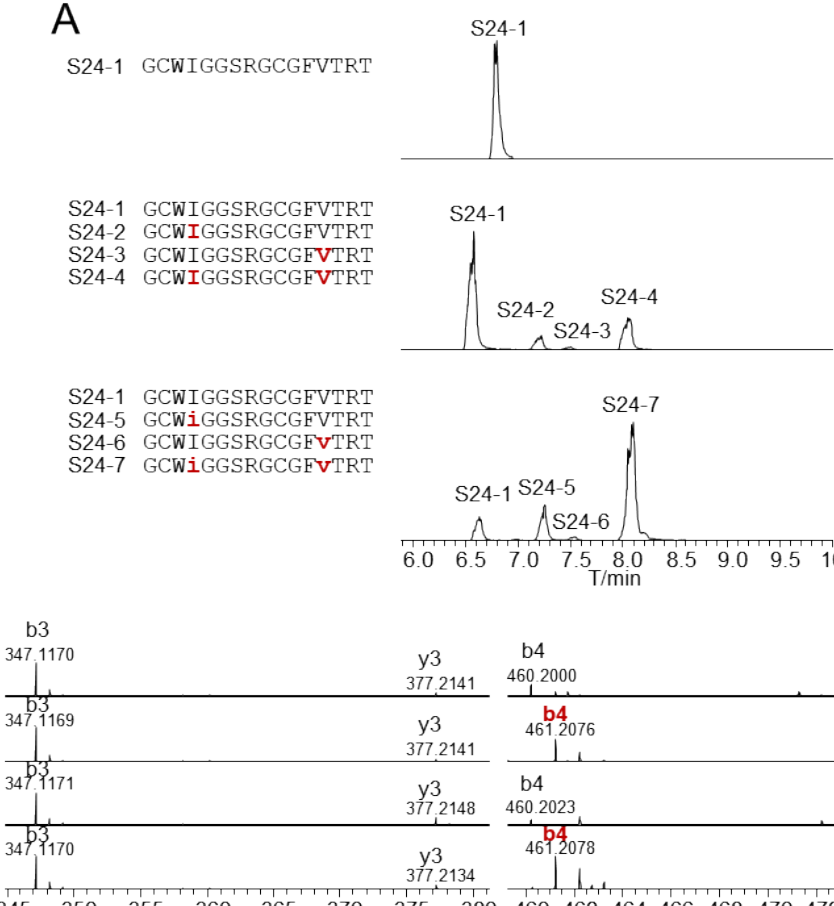

S24-2 S24-4

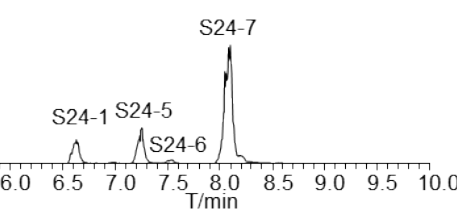

$I_{478}^{2891}$ S24-5G C W $\left.\left.\right|_{i}\right|_{G} ^{b}$

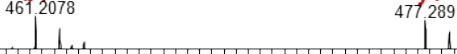

G S R G C G $\left.\left.\left.\right|_{\mathrm{y} 5 \mathrm{y}^{4} \mathrm{y} 3} ^{\mathrm{F} 3}\right|^{\mathrm{V}}\right|^{\mathrm{T}} \mathrm{T}$

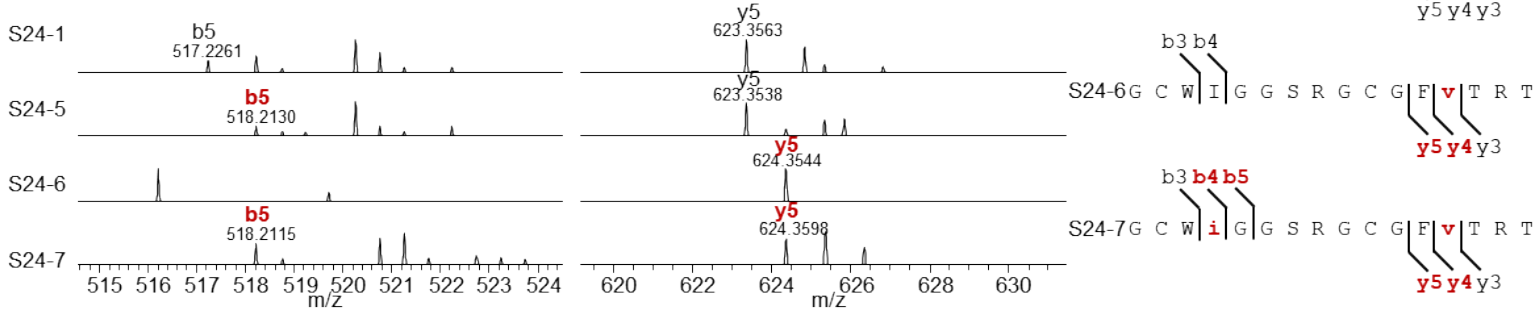

Figure S24. Characterization of selectively labeled products from co-expression of $o s p A-A 5 G+o s p D$ using ODIS. His ${ }_{6}-\mathrm{OspA}$ variant was cleaved with Factor Xa. (A) EICs of unmodified core peptides (S24-1), core peptides epimerized in TB (S24-2 to S24-4), and epimerized in $\mathrm{D}_{2} \mathrm{O}$-based medium (S24-5 to S24-7). (B) MS for parent ions of S24-1, S24-5 to S24-7 acquired by LC-MS full-scan of ODIS experiment. (C) $\mathrm{MS}^{2}$ derived from parent ions of S24-1, S24-5 to S24-7 acquired by LC-MS 2 (PRM) of ODIS experiments. Bold (red) residues indicate epimerized residues. Small letter (red) indicates selective labeling with deuterium at the corresponding epimerized residue. 


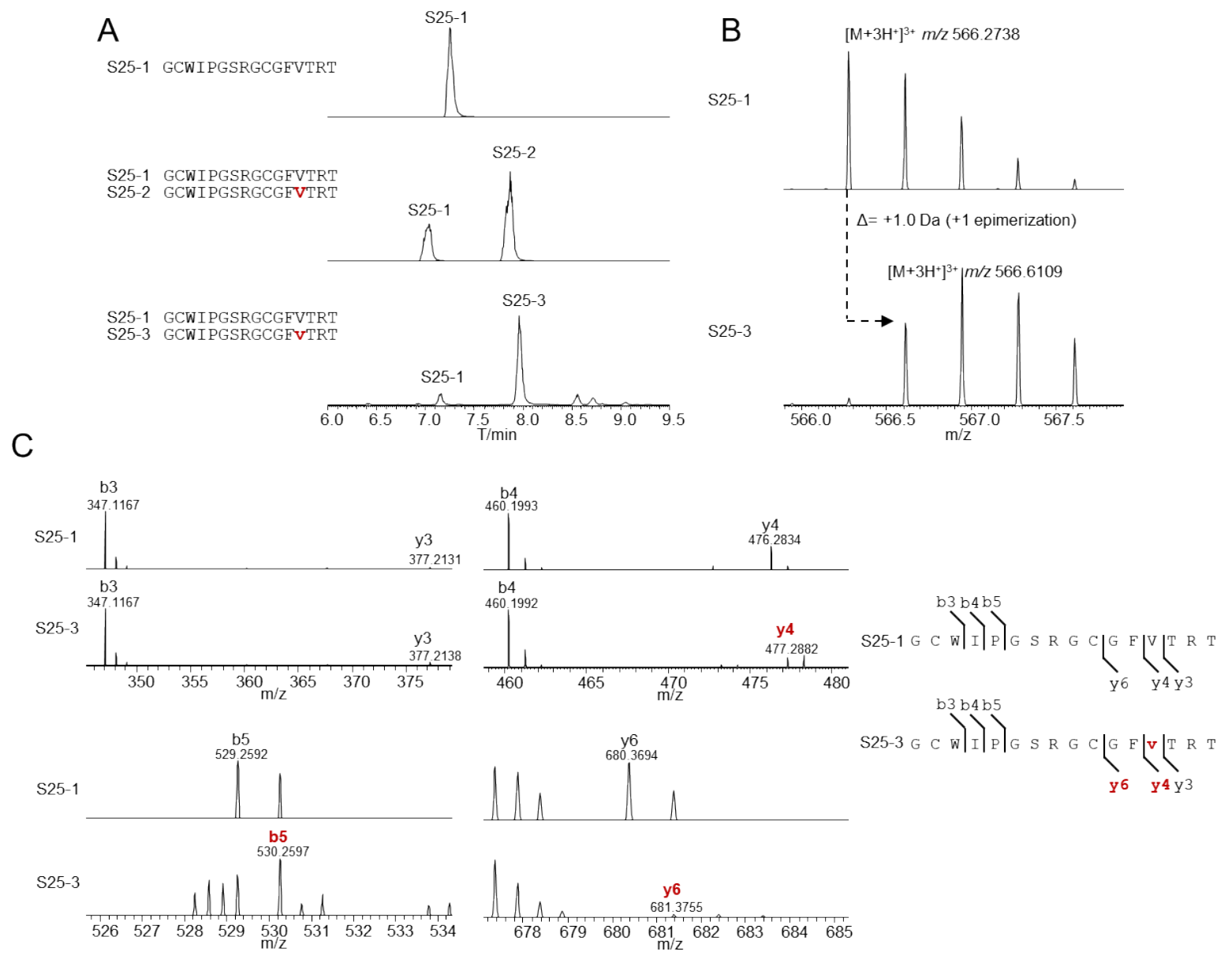

S25. Characterization of selectively labeled products from co-expression of $o s p A-A 5 P+o s p D$ using ODIS. His ${ }_{6}$-OspA variant was cleaved with Factor Xa. (A) EICs of unmodified core peptides (S25-1), core peptides epimerized in TB (S25-2), and epimerized in $\mathrm{D}_{2} \mathrm{O}-$ based medium (S25-3). (B) MS for parent ions of S25-1 and S25-3 acquired by LC-MS full-scan of ODIS experiment. (C) MS ${ }^{2}$ derived from parent ions of S25-1 and S25-3 acquired by LC-MS ${ }^{2}$ (PRM) of ODIS experiments. Bold (red) residues indicate epimerized residues. Small letter (red) indicates selective labeling with deuterium at the corresponding epimerized residue. 


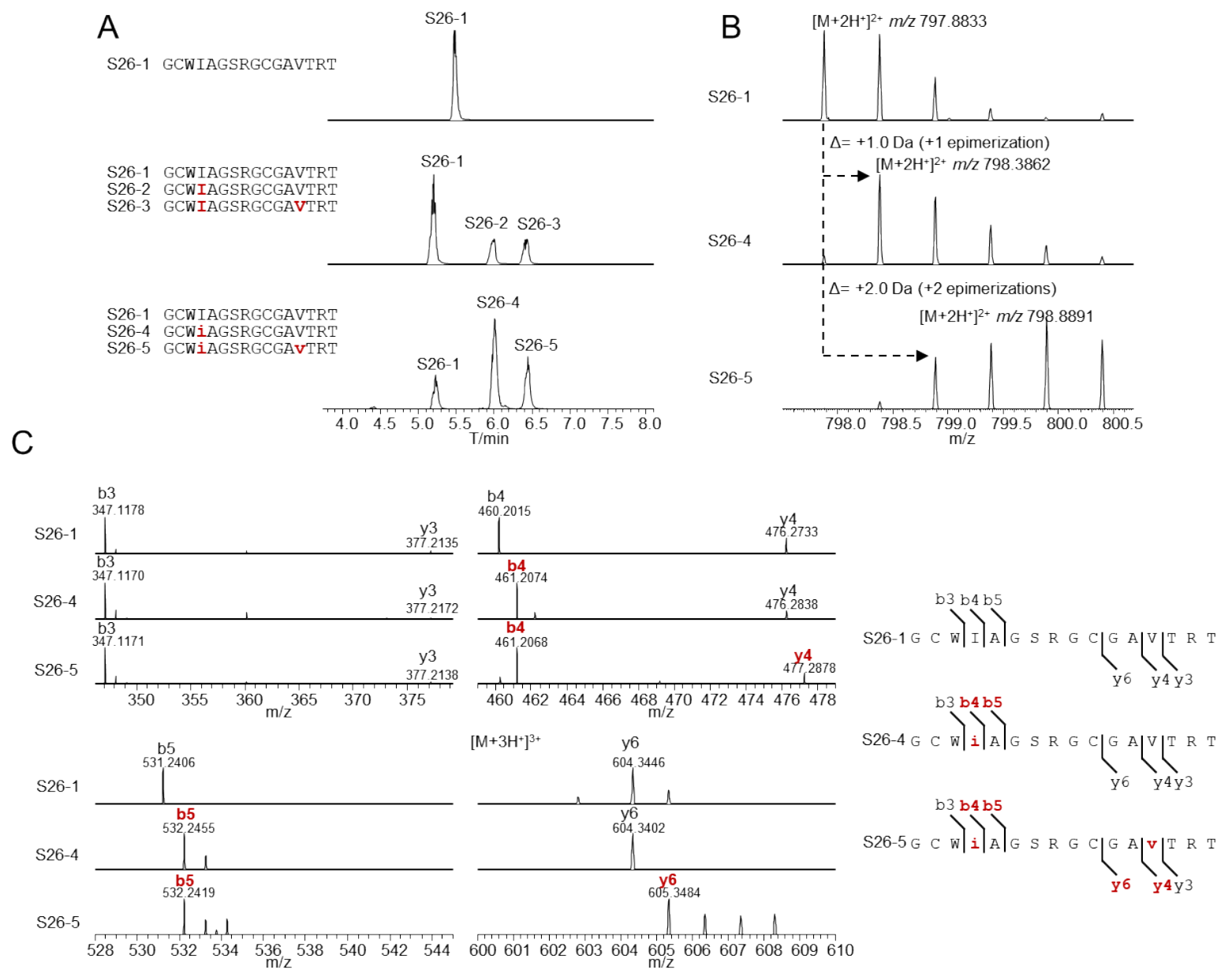

Figure S26. Characterization of selectively labeled products from co-expression of osp $A-F 12 A+o s p D$ using ODIS. His 6 -OspA variant was cleaved with Factor Xa. (A) EICs of unmodified core peptides (S26-1), core peptides epimerized in TB (S26-2 to S26-3), and epimerized in $\mathrm{D}_{2} \mathrm{O}$-based medium (S26-4 to S26-5). (B) MS for parent ions of S26-1, S26-4 to S26-5 acquired by LC-MS full-scan of ODIS experiment. (C) $\mathrm{MS}^{2}$ derived from parent ions of S26-1, S26-4 to S26-5 acquired by LC-MS² (PRM) of ODIS experiments. Bold (red) residues indicate epimerized residues. Small letter (red) indicates selective labeling with deuterium at the corresponding epimerized residue. 


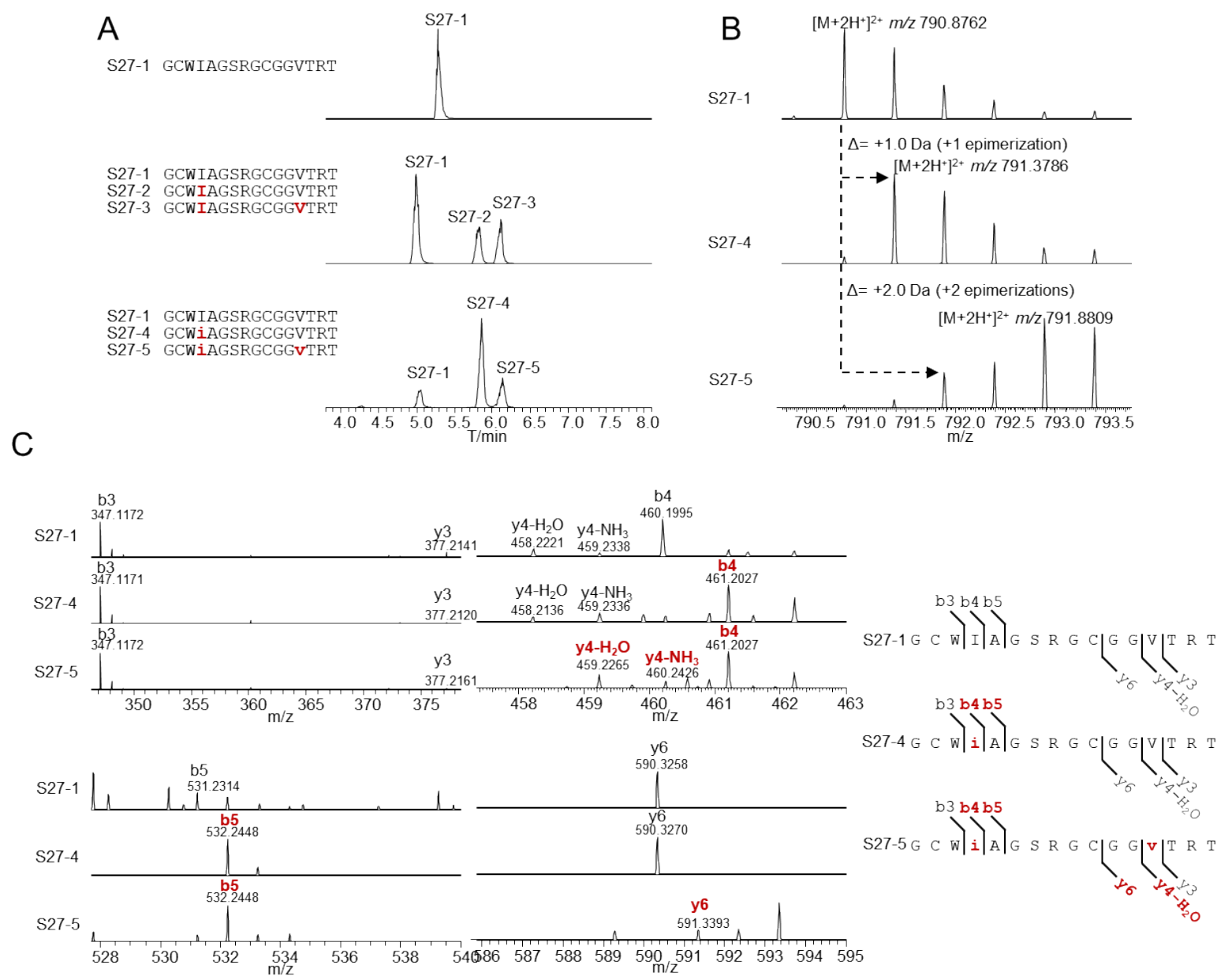

Figure S27. Characterization of selectively labeled products from co-expression of $o s p A-F 12 G+o s p D$ using ODIS. His 6 -OspA variant was cleaved with Factor Xa. (A) EICs of unmodified core peptides (S27-1), core peptides epimerized in TB (S27-2 to S27-3), and epimerized in $\mathrm{D}_{2} \mathrm{O}$-based medium (S27-4 to S27-5). (B) MS for parent ions of S27-1, S27-4 to S27-5 acquired by LC-MS full-scan of ODIS experiment. (C) $\mathrm{MS}_{2}$ derived from parent ions of S27-1, S27-4 to S27-5 acquired by LC-MS 2 (PRM) of ODIS experiments. Bold (red) residues indicate epimerized residues. Small letter (red) indicates selective labeling with deuterium at the corresponding epimerized residue. 


\section{A}

S28-1 GCWIAGSRGCGPVTRT

S28-1 GCWIAGSRGCGPVTRT S28-2 GCWIAGSRGCGPVTRT S28-3 GCWIAGSRGCGPVTRT S28-3 GCWIAGSRGCGPVTRT S28-4 GCWIAGSRGCGPVTRT

S28-1 GCWIAGSRGCGPVTRT S28-5 GCWIAGSRGCGPVTRT S28-6 GCW S28-7 GCWiGGSRGCGEVTRT

\section{C}
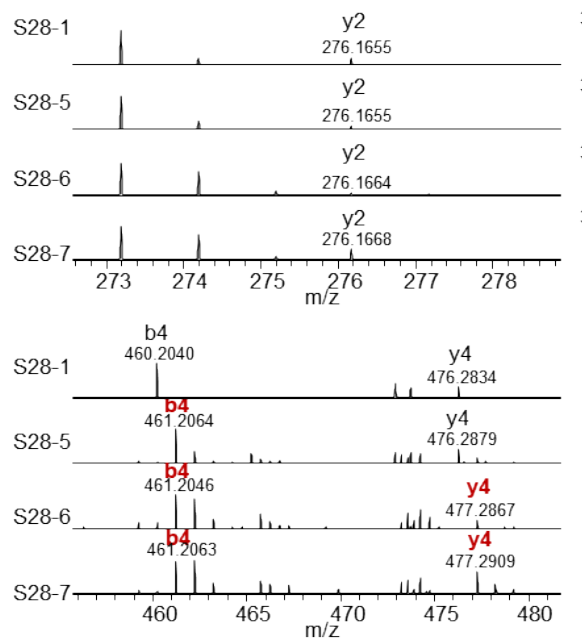
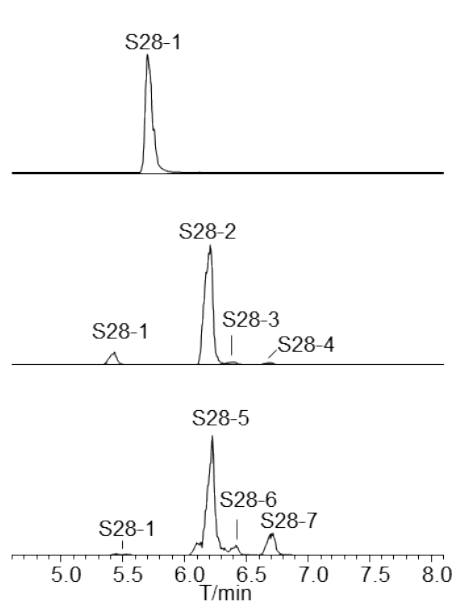

b3

347. $1170 \quad$ y3
B

S28-1

S28-5

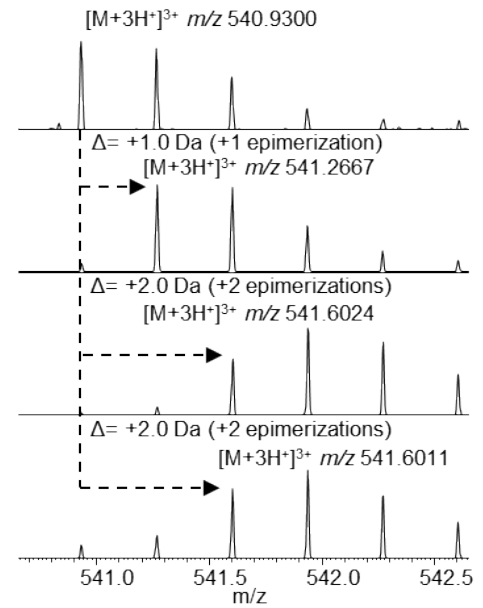

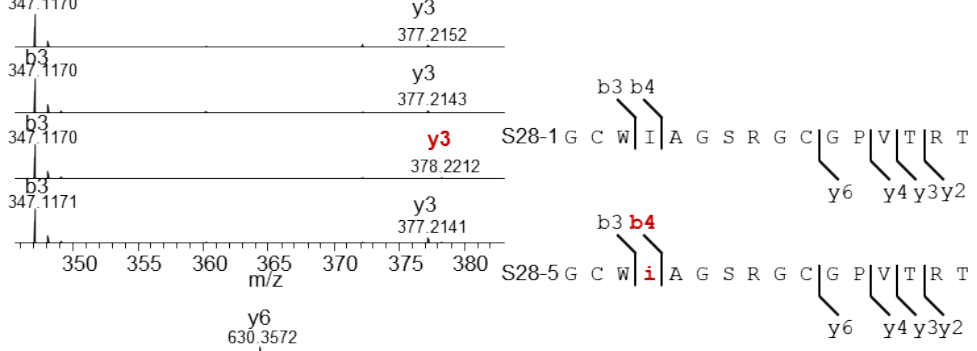
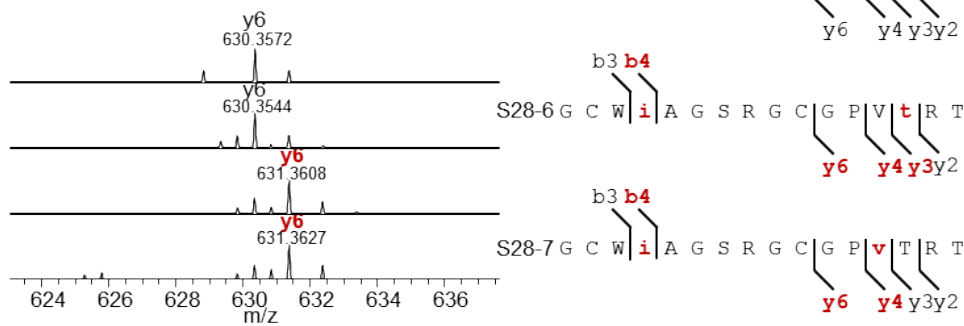

Figure S28. Characterization of selectively labeled products from co-expression of $o s p A-F 12 P+o s p D$ using ODIS. His ${ }_{6}$-OspA variant was cleaved with Factor Xa. (A) EICs of unmodified core peptides (S28-1), core peptides epimerized in TB (S28-2 to S28-4), and epimerized in $\mathrm{D}_{2} \mathrm{O}$-based medium (S28-5 to S28-7). (B) MS for parent ions of S28-1, S28-5 to S28-7 acquired by LC-MS full-scan of ODIS experiment. (C) $\mathrm{MS}^{2}$ derived from parent ions of S28-1, S28-5 to S28-7 acquired by LC-MS ${ }^{2}$ (PRM) of ODIS experiments. Bold (red) residues indicate epimerized residues. Small letter (red) indicates selective labeling with deuterium at the corresponding epimerized residue. 
A

C

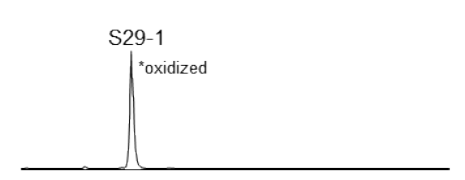

S29-1 GCWIAGSRGCGFVART S29-2 GCWIAGSRGCGEVART S29-3 GCWIAGSRGCGEVART S29-4 GCWIAGSRGCGFVART

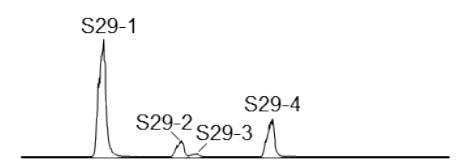

S29-1 GCWIAGSRGCGFVART 29-5 GCWIAGSRGCGFVAR S29-7 GCWiGGSRGCGFvART

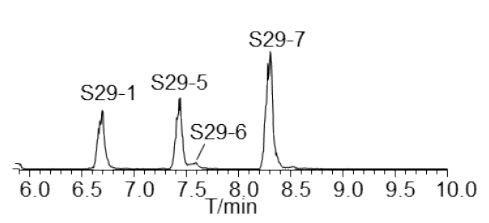

B

(

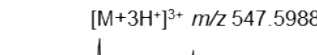

$29-5$

S29-6

S29-7

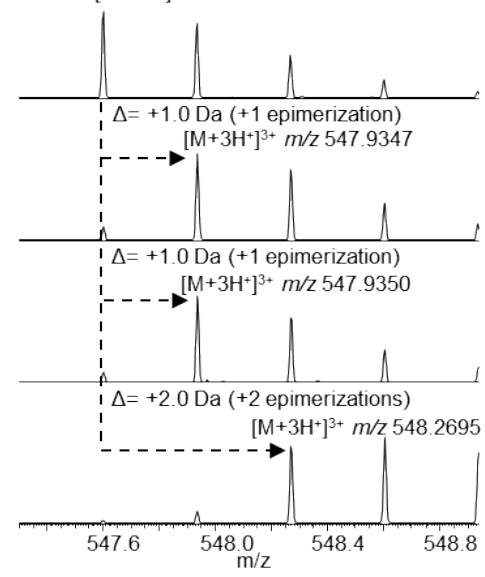

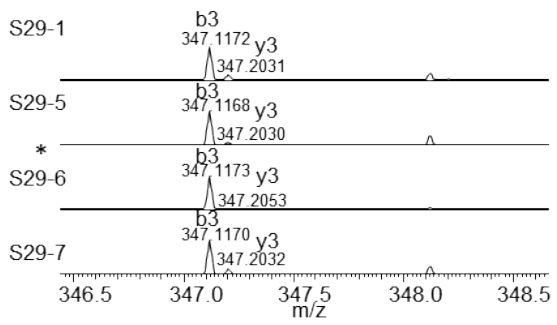
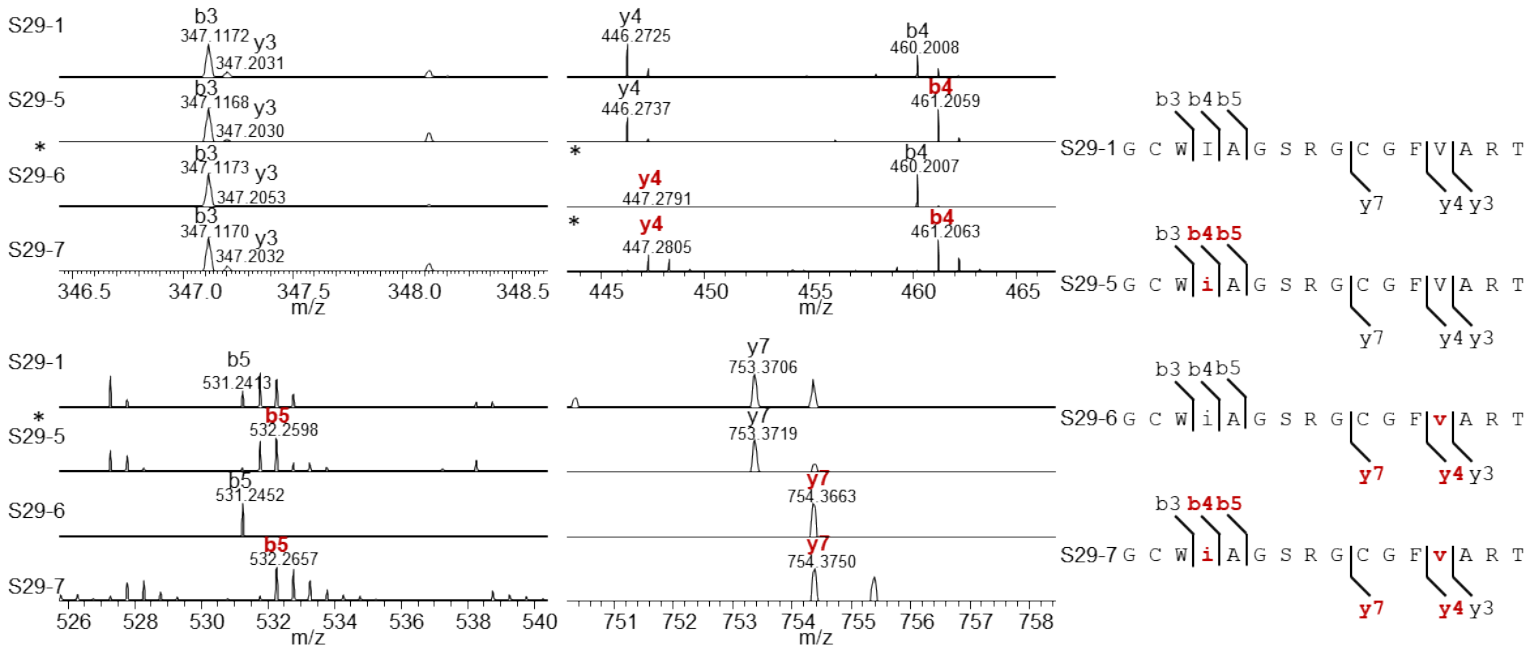

Figure S29. Characterization of selectively labeled products from co-expression of $o s p A-T 14 A+o s p D$ using ODIS. His 6 -OspA variant was cleaved with Factor Xa. (A) EICs of unmodified core peptides (S29-1), core peptides epimerized in TB (S29-2 to S29-4), and epimerized in $\mathrm{D}_{2} \mathrm{O}$-based medium (S29-5 to S29-7). (B) MS for parent ions of S29-1, S29-5 to S29-7 acquired by LC-MS full-scan of ODIS experiment. (C) $\mathrm{MS}^{2}$ derived from parent ions of S29-1, S29-5 to S29-7 acquired by LC-MS 2 (PRM) of ODIS experiments. Bold (red) residues indicate epimerized residues. Small letter (red) indicates selective labeling with deuterium at the corresponding epimerized residue. Repeated measurements for improved $\mathrm{MS}^{2}$ data are indicated with asterisks. 


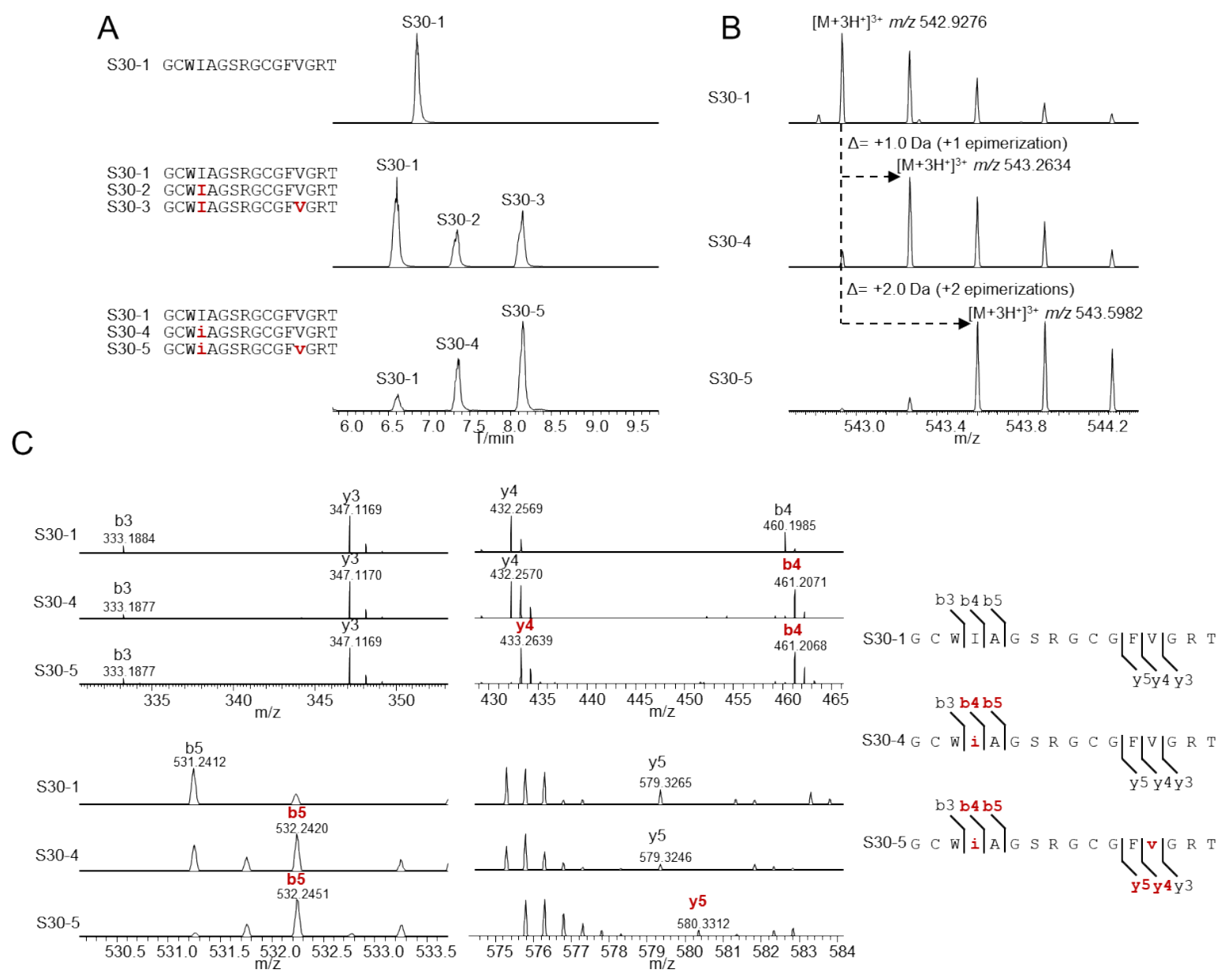

Figure S30. Characterization of selectively labeled products from co-expression of $o s p A-T 14 G+o s p D$ using ODIS. His ${ }_{6}$-OspA variant was cleaved with Factor Xa. (A) EICs of unmodified core peptides (S30-1), core peptides epimerized in TB (S30-2 to S30-3), and epimerized in $\mathrm{D}_{2} \mathrm{O}$-based medium (S30-4 to S30-5). (B) MS for parent ions of S30-1, S30-4 to S30-5 acquired by LC-MS full-scan of ODIS experiment. (C) $\mathrm{MS}^{2}$ derived from parent ions of S30-1, S30-4 to S30-5 acquired by LC-MS ${ }^{2}$ (PRM) of ODIS experiments. Bold (red) residues indicate epimerized residues. Small letter (red) indicates selective labeling with deuterium at the corresponding epimerized residue. 


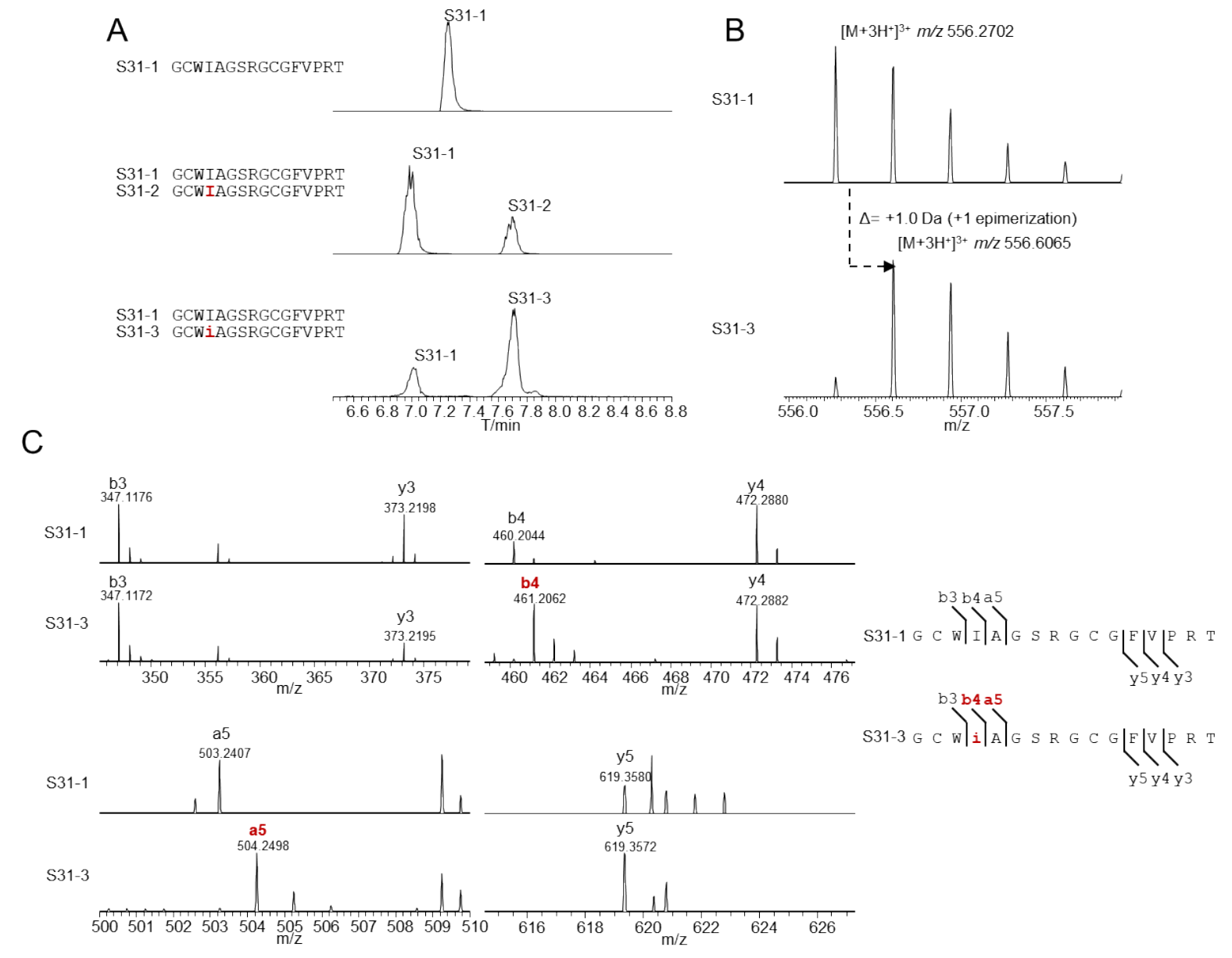

Figure S31. Characterization of selectively labeled products from co-expression of $o s p A-T 14 P+o s p D$ using ODIS. His ${ }_{6}$-OspA variant was cleaved with Factor Xa. (A) EICs of unmodified core peptides (S31-1), core peptides epimerized in TB (S31-2), and epimerized in $\mathrm{D}_{2} \mathrm{O}-$ based medium (S31-3). (B) MS for parent ions of S31-1 and S31-3 acquired by LC-MS full-scan of ODIS experiment. (C) MS ${ }^{2}$ derived from parent ions of S31-1 and S31-3 acquired by LC-MS ${ }^{2}$ (PRM) of ODIS experiments. Bold (red) residues indicate epimerized residues. Small letter (red) indicates selective labeling with deuterium at the corresponding epimerized residue. 
A

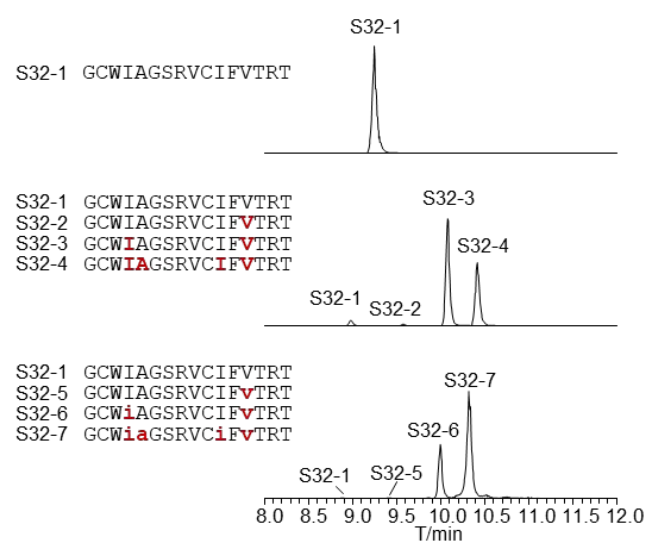

B

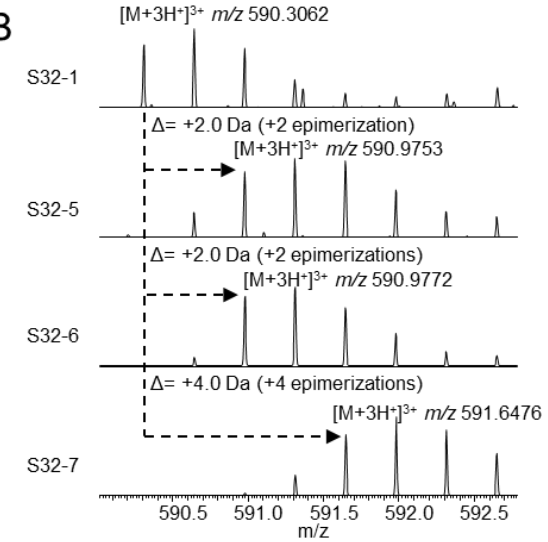

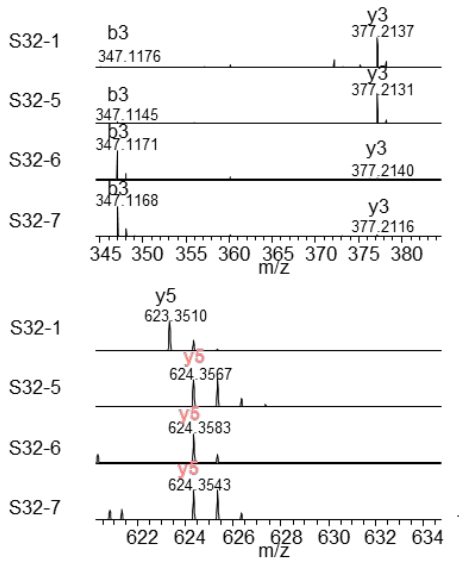
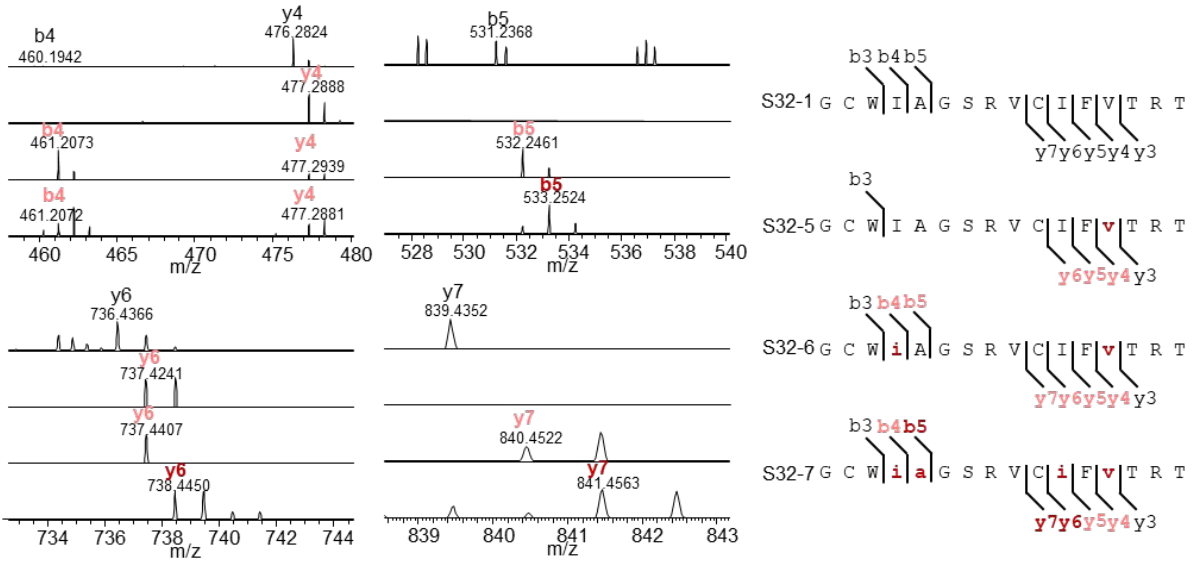

Figure S32. Characterization of selectively labeled products from co-expression of $o s p A-G 9 V G 11 I+o s p D$ using ODIS. His ${ }_{6}$-OspA variant was cleaved with Factor Xa. (A) EICs of unmodified core peptides (S32-1), core peptides epimerized in TB (S32-2 to S32-4), and epimerized in $\mathrm{D}_{2} \mathrm{O}$-based medium (S32-5 to S32-7). (B) MS for parent ions of S32-1, S32-5 to S32-7 acquired by LC-MS full-scan of ODIS experiment. (C) $\mathrm{MS}^{2}$ derived from parent ions of S32-1, S32-5 to S32-7 acquired by LC-MS2 (PRM) of ODIS experiments. Bold (red) residues indicate epimerized residues. Small letter (red) indicates selective labeling with deuterium at the corresponding epimerized residue. Rose-colored b/y ions have mass shift of +1.0 Da, red b/y ions of +2.0 Da. 
A

S33-1 GCWIAGSRG

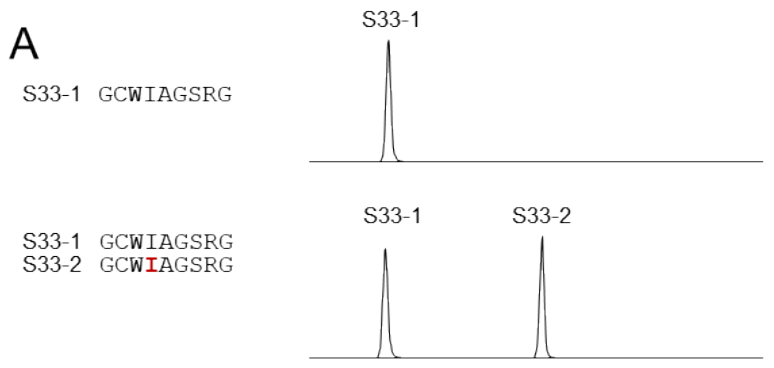

S33-1 GCWIAGSRG S33-3 GCWiAGSRG

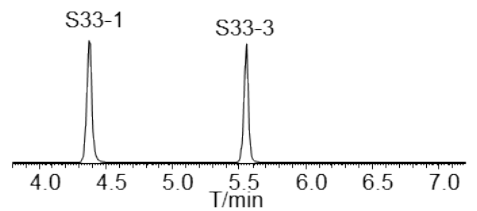

B

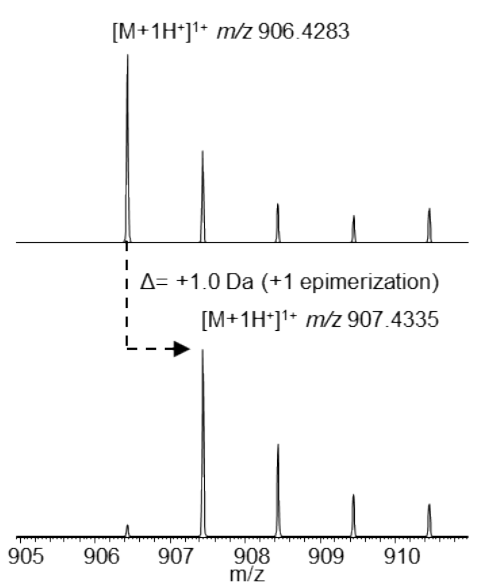

S33-

S33-3

C
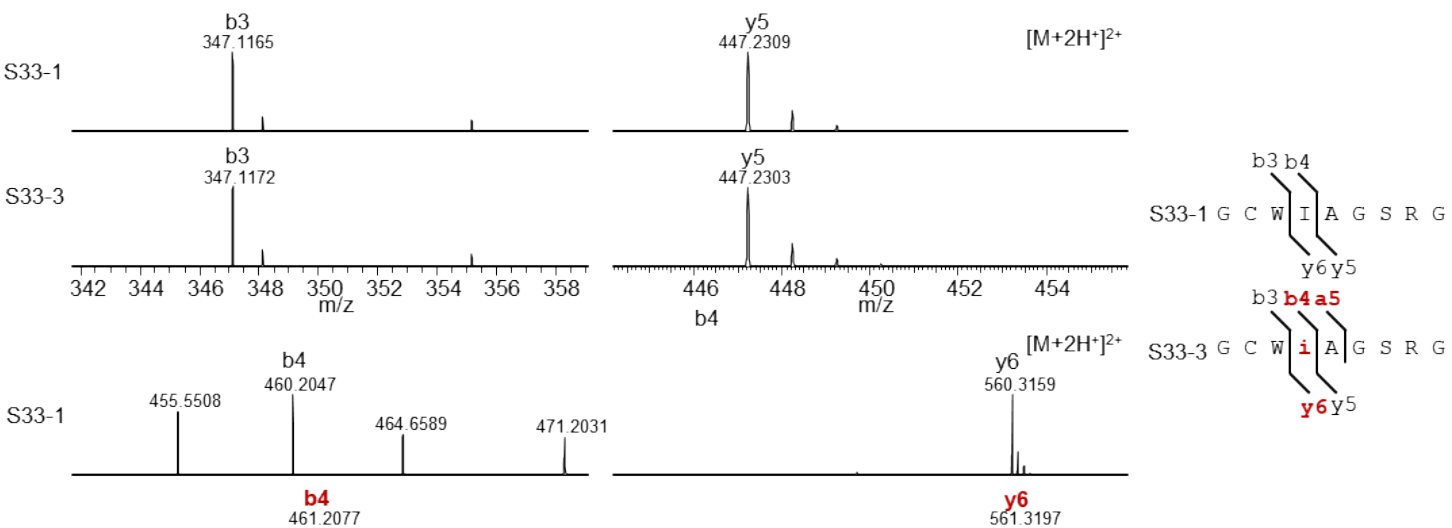

S33-3
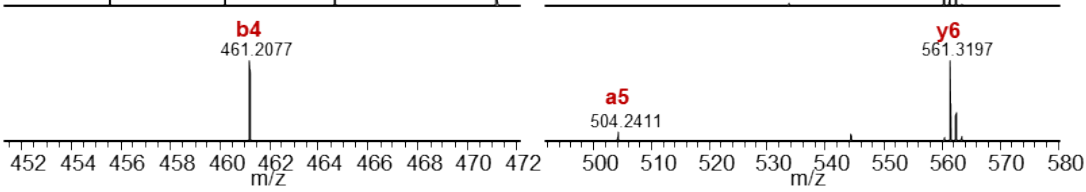

Figure S33. Characterization of selectively labeled products from co-expression of truncated $o s p A-C 10 X+o s p D$ using ODIS. His 6 -OspA variant was cleaved with Factor Xa. (A) EICs of unmodified core peptides (S33-1), core peptides epimerized in TB (S33-2), and epimerized in $\mathrm{D}_{2} \mathrm{O}$-based medium (S33-3). (B) MS for parent ions of S33-1 and S33-3 acquired by LC-MS full-scan of ODIS experiment. (C) MS derived from parent ions of S33-1 and S33-3 acquired by LC-MS ${ }^{2}$ (PRM) of ODIS experiments. Bold (red) residues indicate epimerized residues. Small letter (red) indicates selective labeling with deuterium at the corresponding epimerized residue. 
A

S34-1 GCWIAG

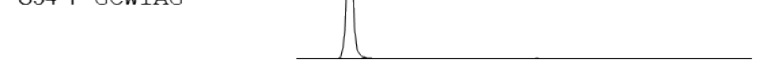

$\begin{array}{ll}\text { S34-1 } & \text { GCWIAG } \\ \text { S34-2 } & \text { GCWIAG }\end{array}$

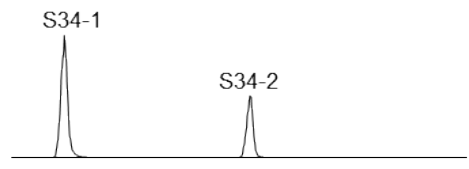

S34-1 GCWIAG S34-3 GCWiAG

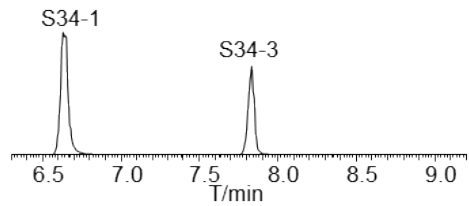

$\mathrm{B}$

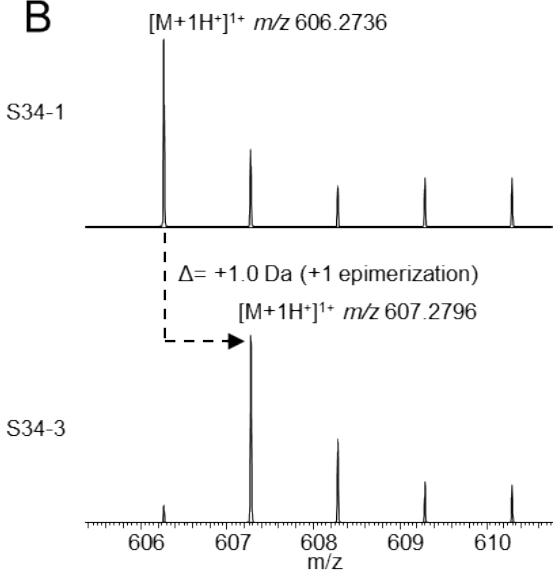

C
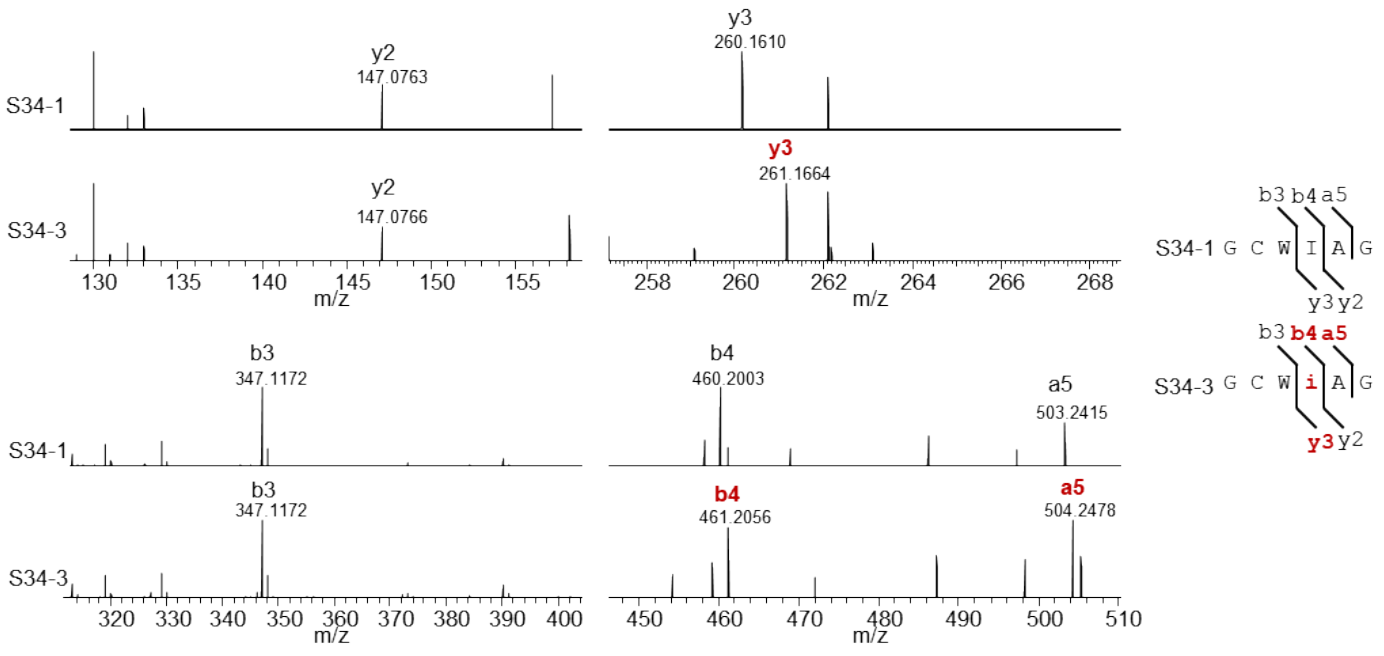

Figure S34. Characterization of selectively labeled products from co-expression of truncated $o s p A-S 7 X+o s p D$ using ODIS. His 6 -OspA variant was cleaved with Factor Xa. (A) EICs of unmodified core peptides (S34-1), core peptides epimerized in TB (S34-2), and epimerized in $\mathrm{D}_{2} \mathrm{O}$-based medium (S34-3). (B) MS for parent ions of S34-1 and S34-3 acquired by LC-MS full-scan of ODIS experiment. (C) MS ${ }^{2}$ derived from parent ions of S34-1 and S34-3 acquired by LC-MS² (PRM) of ODIS experiments. Bold (red) residues indicate epimerized residues. Small letter (red) indicates selective labeling with deuterium at the corresponding epimerized residue. 
A

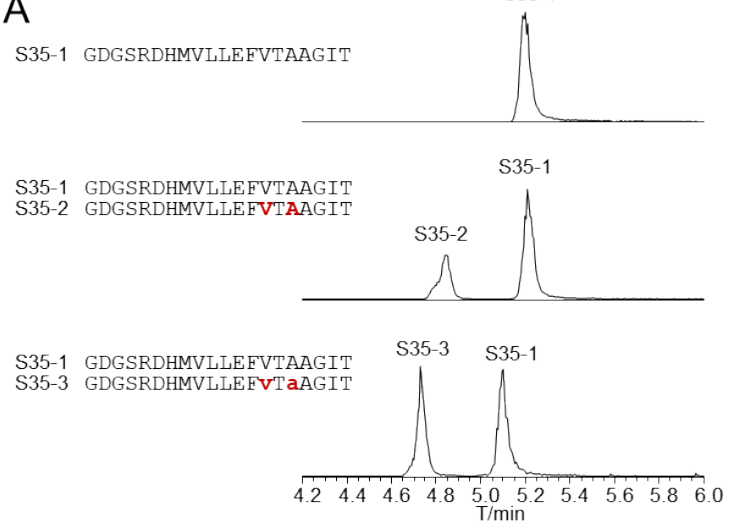

B

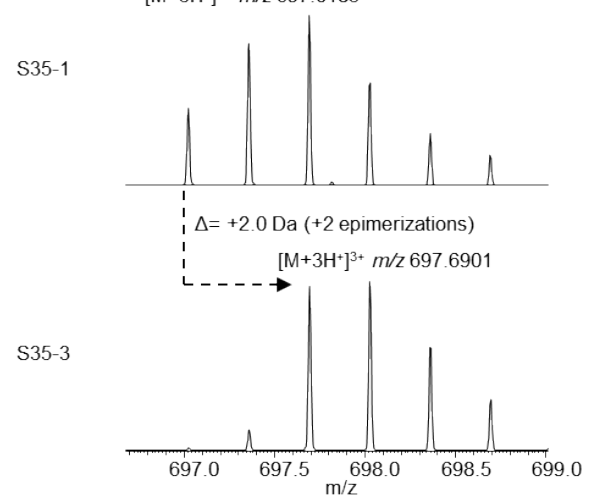

C

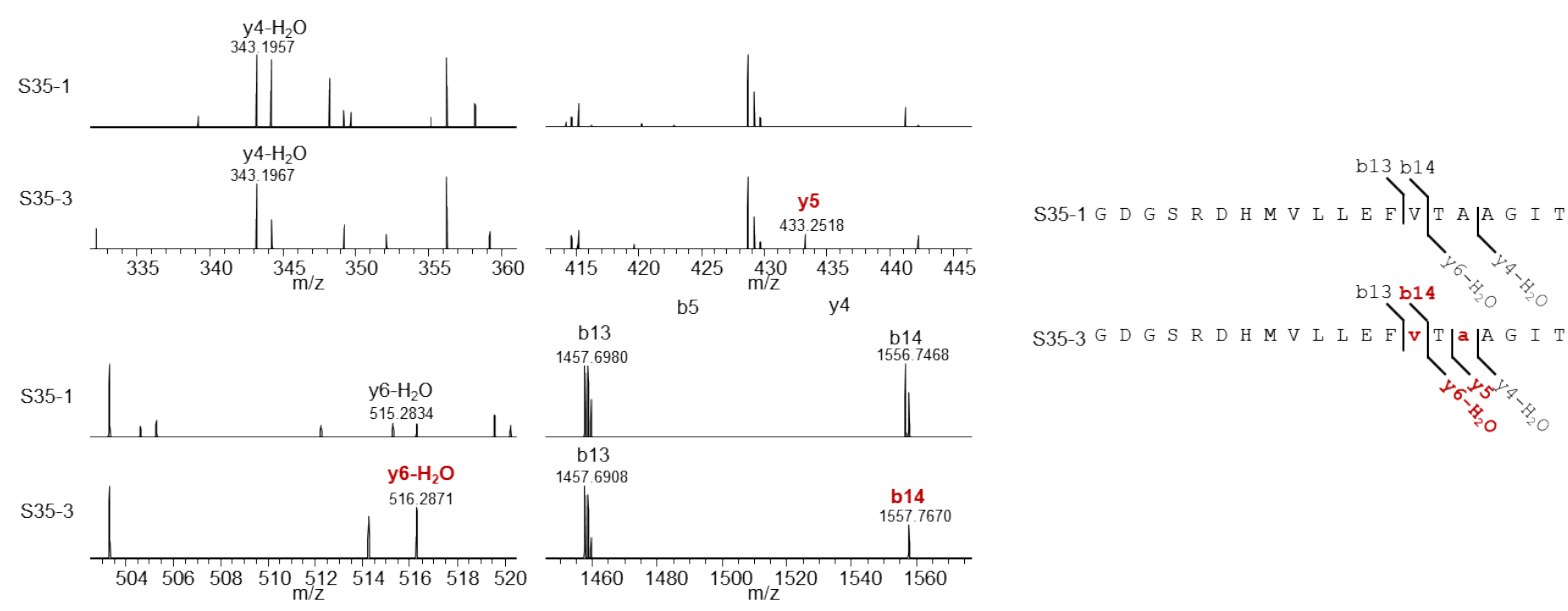

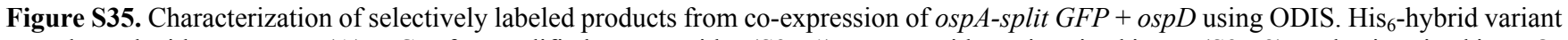
was cleaved with Factor Xa. (A) EICs of unmodified core peptides (S35-1), core peptides epimerized in TB (S35-2), and epimerized in $\mathrm{D}_{2} \mathrm{O}-$ based medium (S35-3). (B) MS for parent ions of S35-1 and S35-3 acquired by LC-MS full-scan of ODIS experiment. (C) MS ${ }^{2}$ derived from parent ions of S35-1 and S35-3 acquired by LC-MS 2 (PRM) of ODIS experiments. Bold (red) residues indicate epimerized residues. Small letter (red) indicates selective labeling with deuterium at the corresponding epimerized residue. 


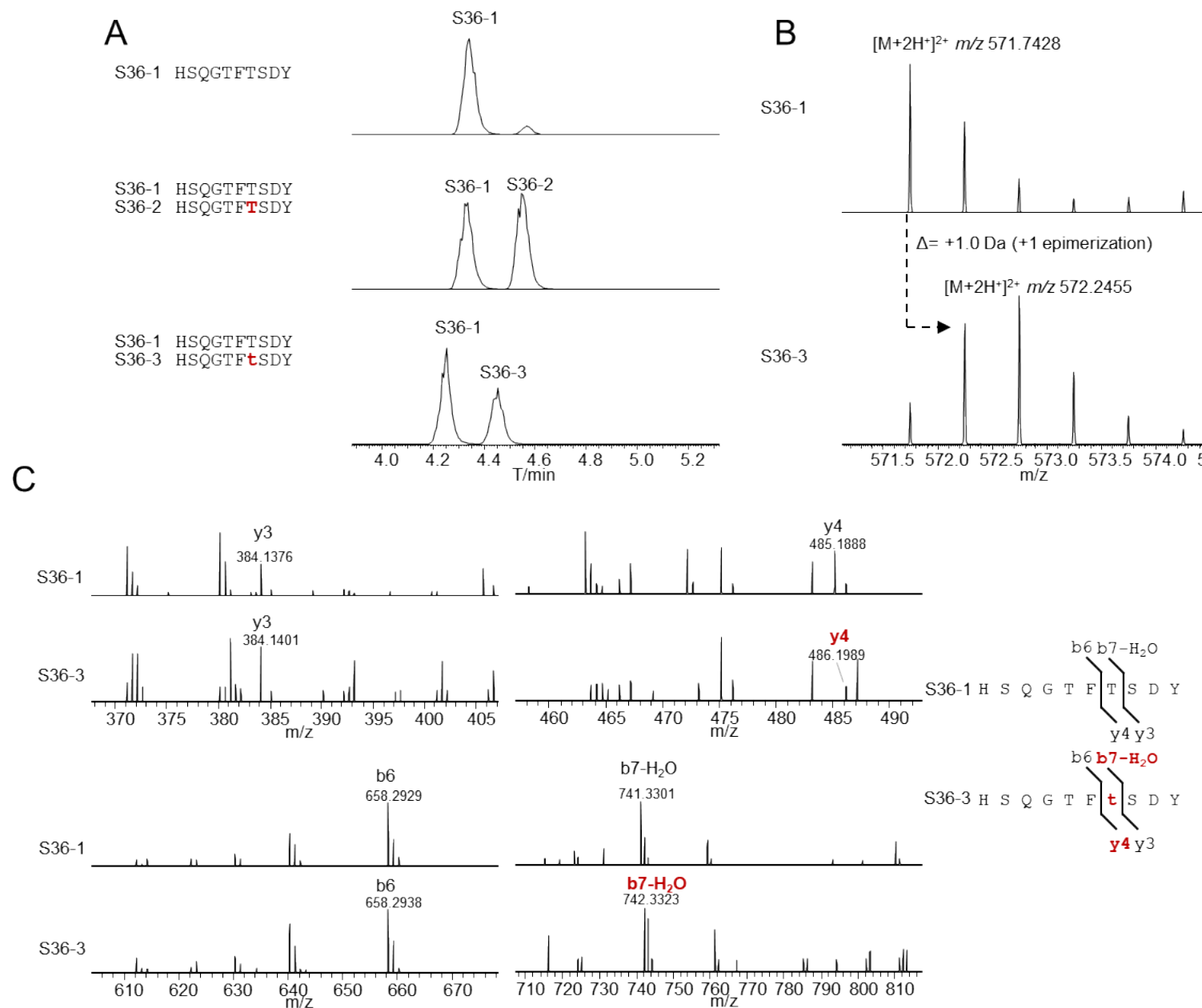

Figure S36. Characterization of selectively labeled products from co-expression of ospA-glucagon ${ }_{(1-10)}+o_{\text {sp }} D$ using ODIS. His 6 -hybrid variant was cleaved with Factor Xa. (A) EICs of unmodified core peptides (S36-1), core peptides epimerized in TB (S36-2), and epimerized in $\mathrm{D}_{2} \mathrm{O}$-based medium (S36-3). (B) MS for parent ions of S36-1 and S36-3 acquired by LC-MS full-scan of ODIS experiment. (C) MS ${ }^{2}$ derived from parent ions of S36-1 and S36-3 acquired by LC-MS ${ }^{2}$ (PRM) of ODIS experiments. Bold (red) residues indicate epimerized residues. Small letter (red) indicates selective labeling with deuterium at the corresponding epimerized residue. 


\section{A}

S37-1 AQDFVQWLMNT

S37-1 AQDFVQWLMNT
S37-2 AQDFVQWLMNT S37-2 AQDFVQWLMNT
S37-3* AQDFVQWLMNT

S37-1 AQDFVQWLMNT
S37-4 AODFVOWIMNT S37-4 AQDFVQWIMNT
S37-5* AQDFVQWLMNT

C
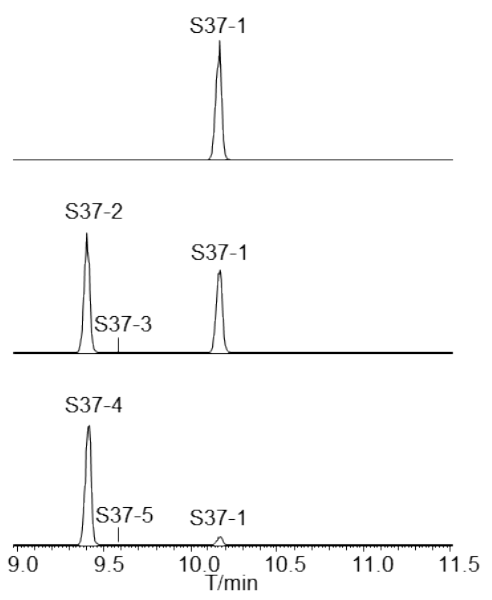

B $\quad[\mathrm{M}+2 \mathrm{H}+]^{2+}+m / 2676.8196$

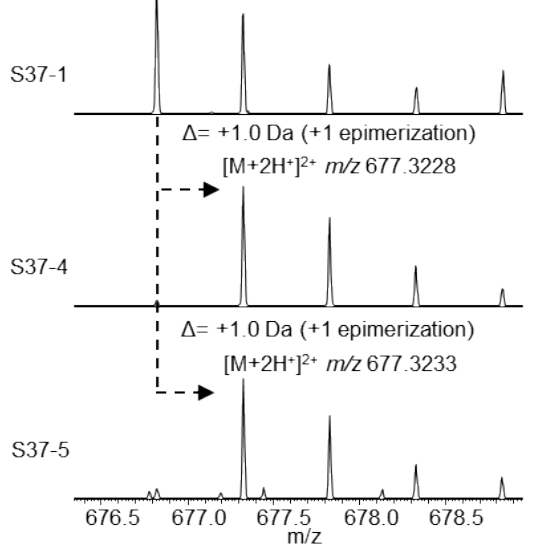

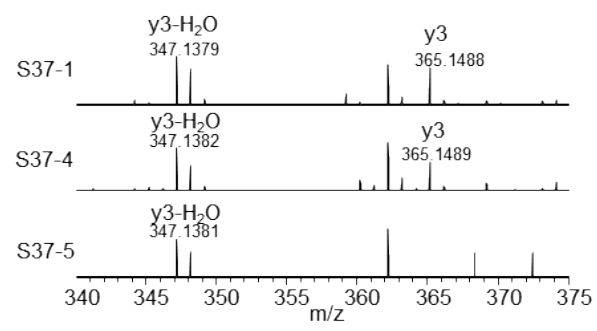
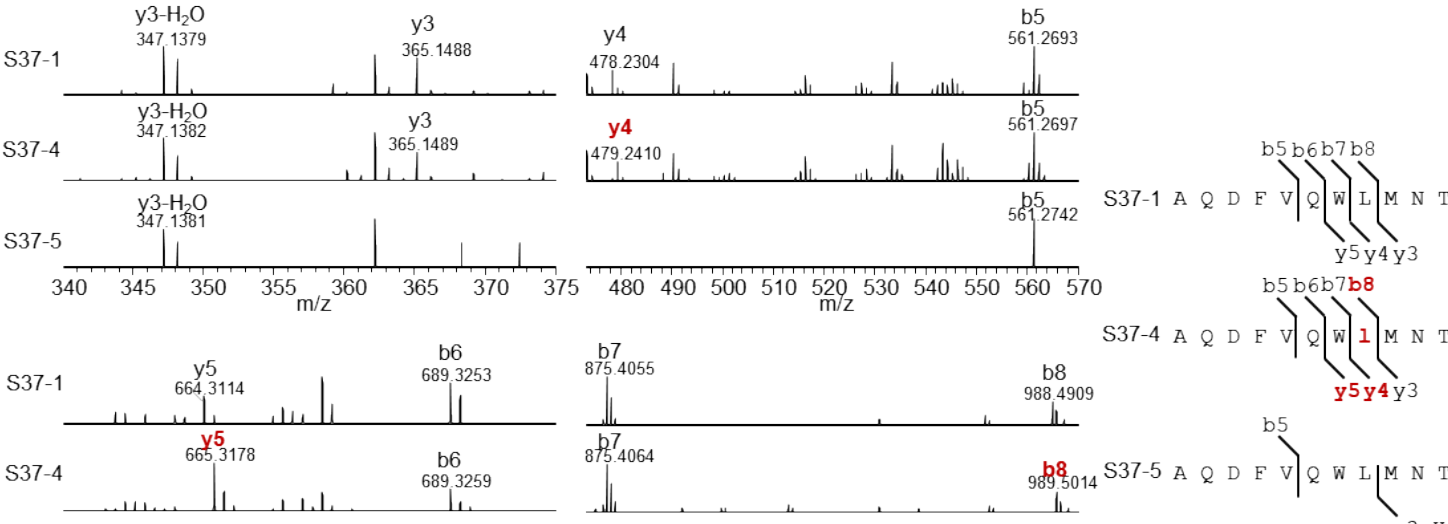

b5
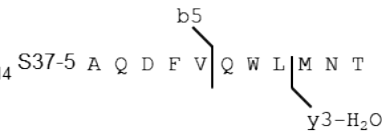

S37-5

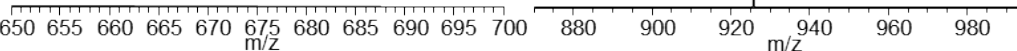

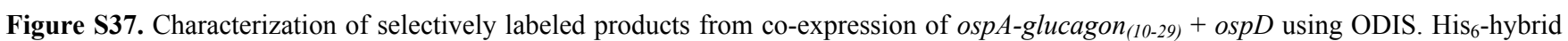
variant was cleaved with trypsin. (A) EICs of unmodified core peptides (S37-1), core peptides epimerized in TB (S37-2 to S37-3), and epimerized in $\mathrm{D}_{2} \mathrm{O}$-based medium (S37-4 to S37-5). (B) MS for parent ions of S37-1, S37-4 to S37-5 acquired by LC-MS full-scan of ODIS experiment. (C) $\mathrm{MS}^{2}$ derived from parent ions of S37-1, S37-4 to S37-5 acquired by LC-MS ${ }^{2}$ (PRM) of ODIS experiments. For S37-5, deuterium label could not be assigned. Bold (red) residues indicate epimerized residues. Small letter (red) indicates selective labeling with deuterium at the corresponding epimerized residue. 


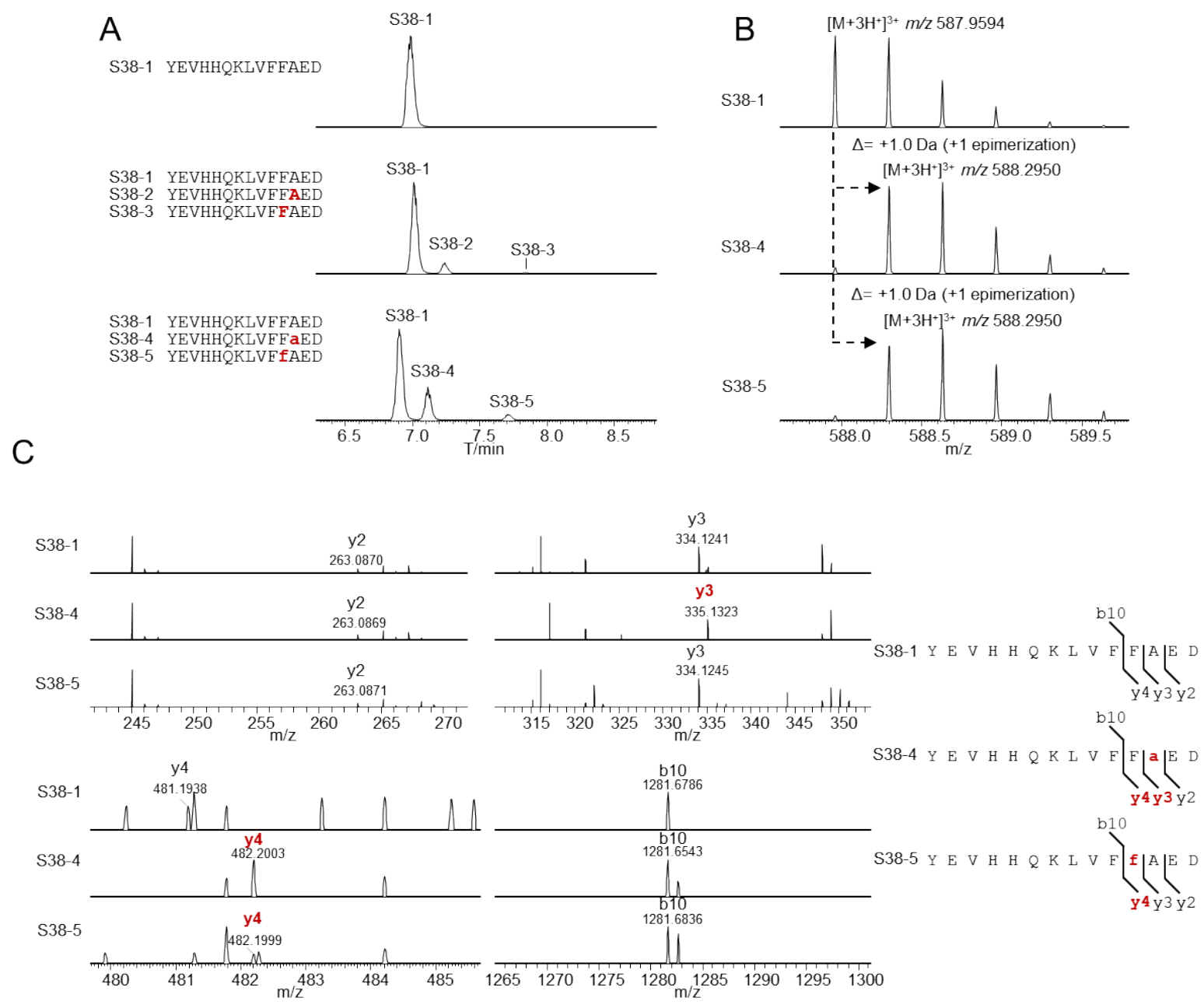

Figure S38. Characterization of selectively labeled products from co-expression of osp A-amyloid $\beta_{(10-23)}+o_{\text {sp }} D$ using ODIS. His 6 -hybrid variant was cleaved with Factor Xa. (A) EICs of unmodified core peptides (S38-1), core peptides epimerized in TB (S38-2 to S38-3), and epimerized in $\mathrm{D}_{2} \mathrm{O}$-based medium (S38-4 to S38-5). (B) MS for parent ions of S38-1, S38-4 to S38-5 acquired by LC-MS full-scan of ODIS experiment. (C) $\mathrm{MS}^{2}$ derived from parent ions of S38-1, S38-4 to S38-5 acquired by LC-MS ${ }^{2}$ (PRM) of ODIS experiments. Bold (red) residues indicate epimerized residues. Small letter (red) indicates selective labeling with deuterium at the corresponding epimerized residue. 
Figure S39. Characterization of selectively labeled products from co-expression of ospA-amyloid $\beta_{(30-41)}+o s p D$ using ODIS. His 6 -hybrid

A

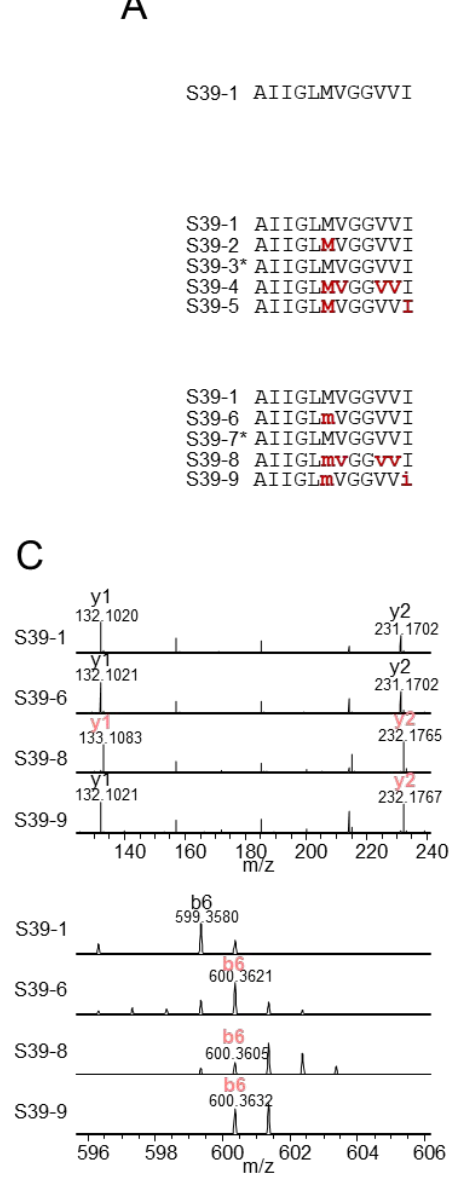

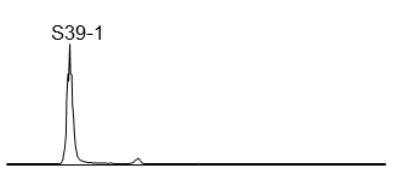
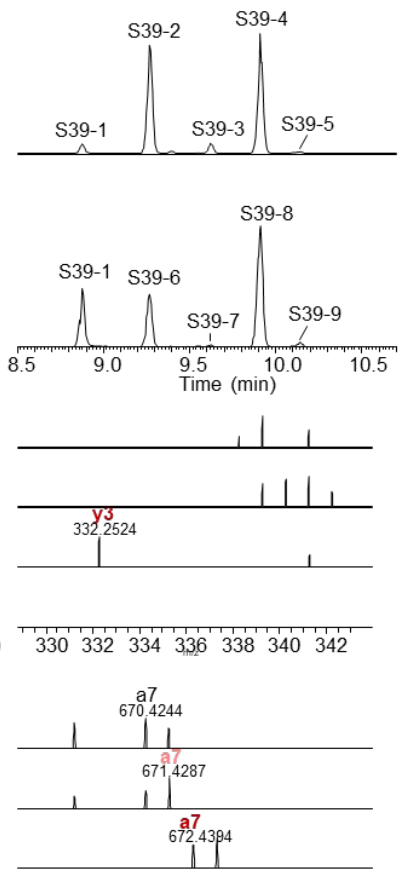

B
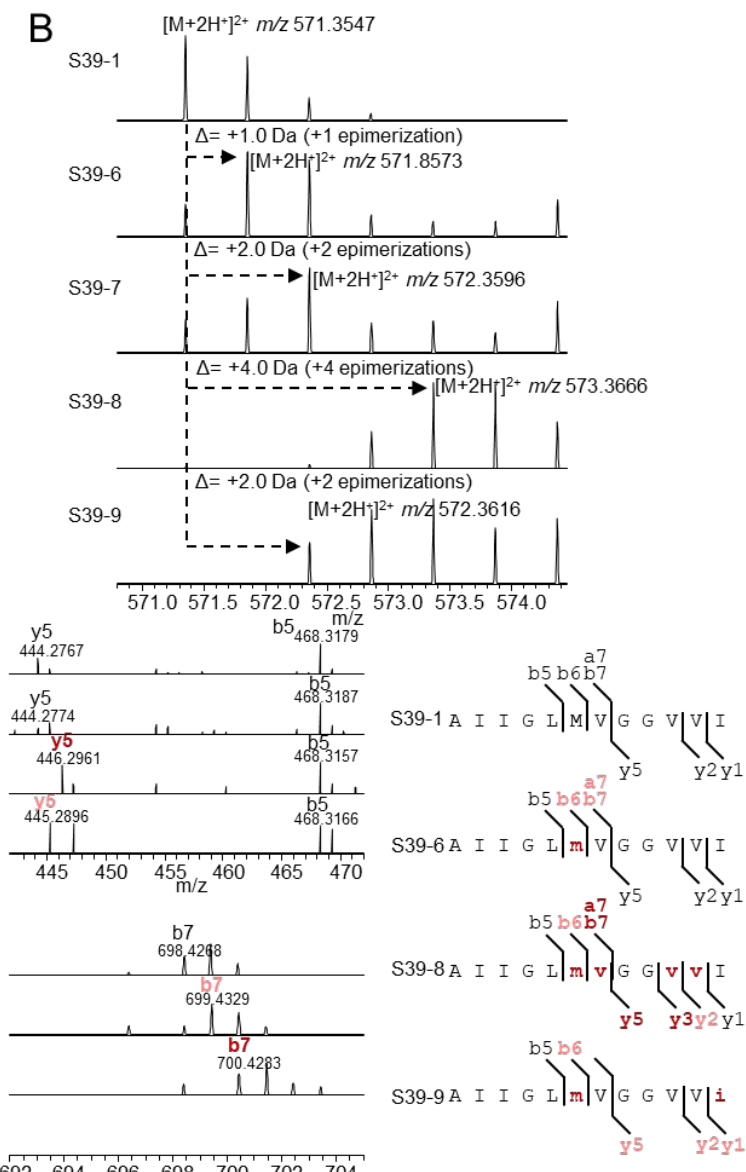

variant was cleaved with Factor Xa. (A) EICs of unmodified core peptides (S39-1), core peptides epimerized in TB (S39-2 to S39-5), and epimerized in $\mathrm{D}_{2} \mathrm{O}$-based medium (S39-6 to S39-9). (B) MS for parent ions of S39-1, S39-6 to S39-9 acquired by LC-MS full-scan of ODIS experiment. (C) MS ${ }^{2}$ derived from parent ions of S39-1, S39-6 and S39-8 to S39-9 acquired by LC-MS2 (PRM) of ODIS experiments. Trace amounts of S39-7 did not allow for $\mathrm{MS}^{2}$ assignment as indicated with an asterisk. Bold (red) residues indicate epimerized residues. Small letter (red) indicates selective labeling with deuterium at the corresponding epimerized residue. Rose-colored a/b/y ions have mass shift of $+1.0 \mathrm{Da}, \mathrm{red} \mathrm{a} / \mathrm{b} / \mathrm{y}$ ions of $+2.0 \mathrm{Da}$. 
Figure S40. Characterization of selectively labeled products from co-expression of $o s p A-G n R H G 6 S+o s p D$ using ODIS. His 6 -hybrid variant

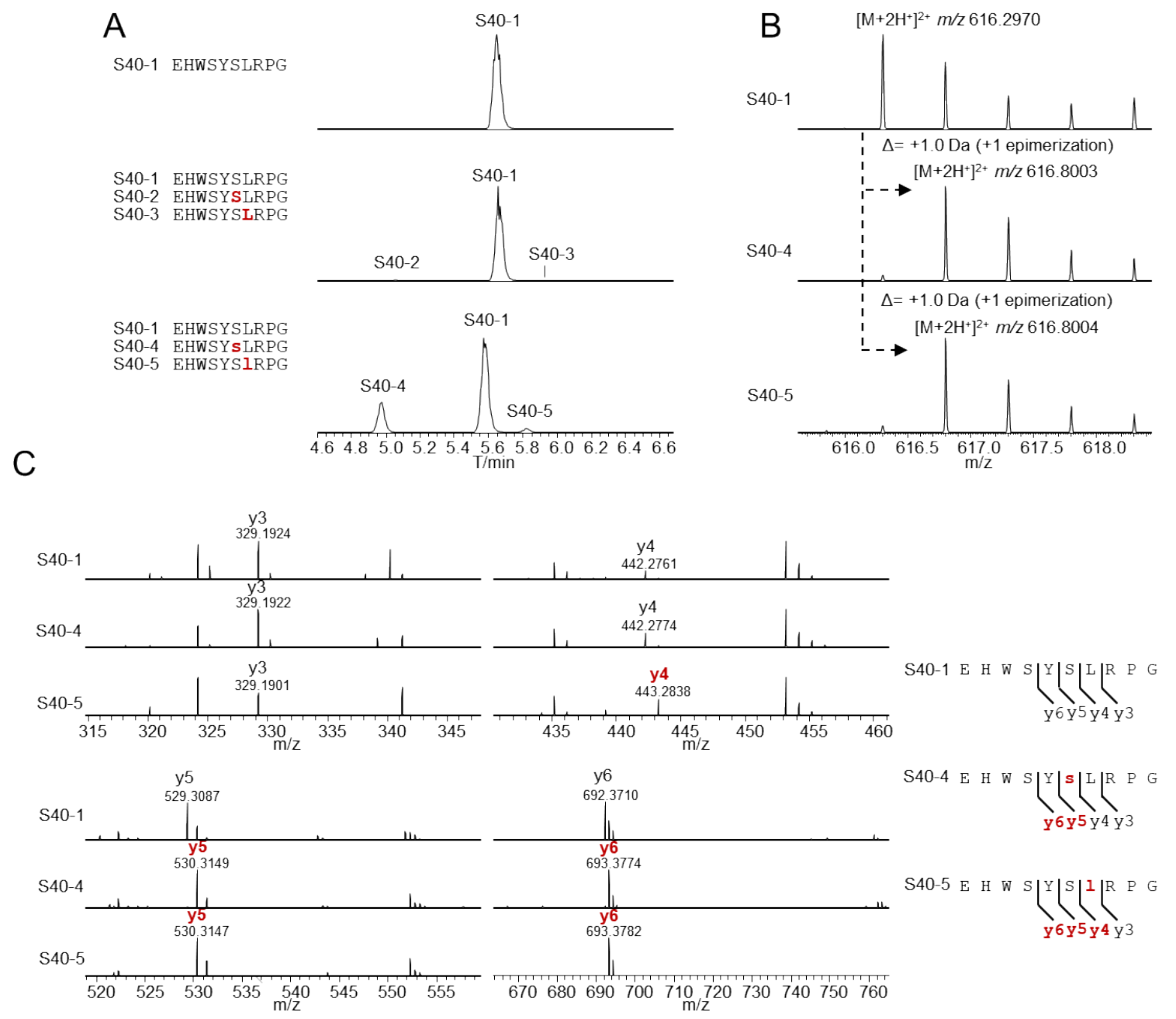

was cleaved with Factor Xa. (A) EICs of unmodified core peptides (S40-1), core peptides epimerized in TB (S40-2 to S40-3), and epimerized in $\mathrm{D}_{2}$ O-based medium (S40-4 to S40-5). (B) MS for parent ions of S40-1, S40-4 to S40-5 acquired by LC-MS full-scan of ODIS experiment. (C) $\mathrm{MS}^{2}$ derived from parent ions of S40-1, S40-4 to S40-5 acquired by LC-MS ${ }^{2}$ (PRM) of ODIS experiments. Bold (red) residues indicate epimerized residues. Small letter (red) indicates selective labeling with deuterium at the corresponding epimerized residue. 

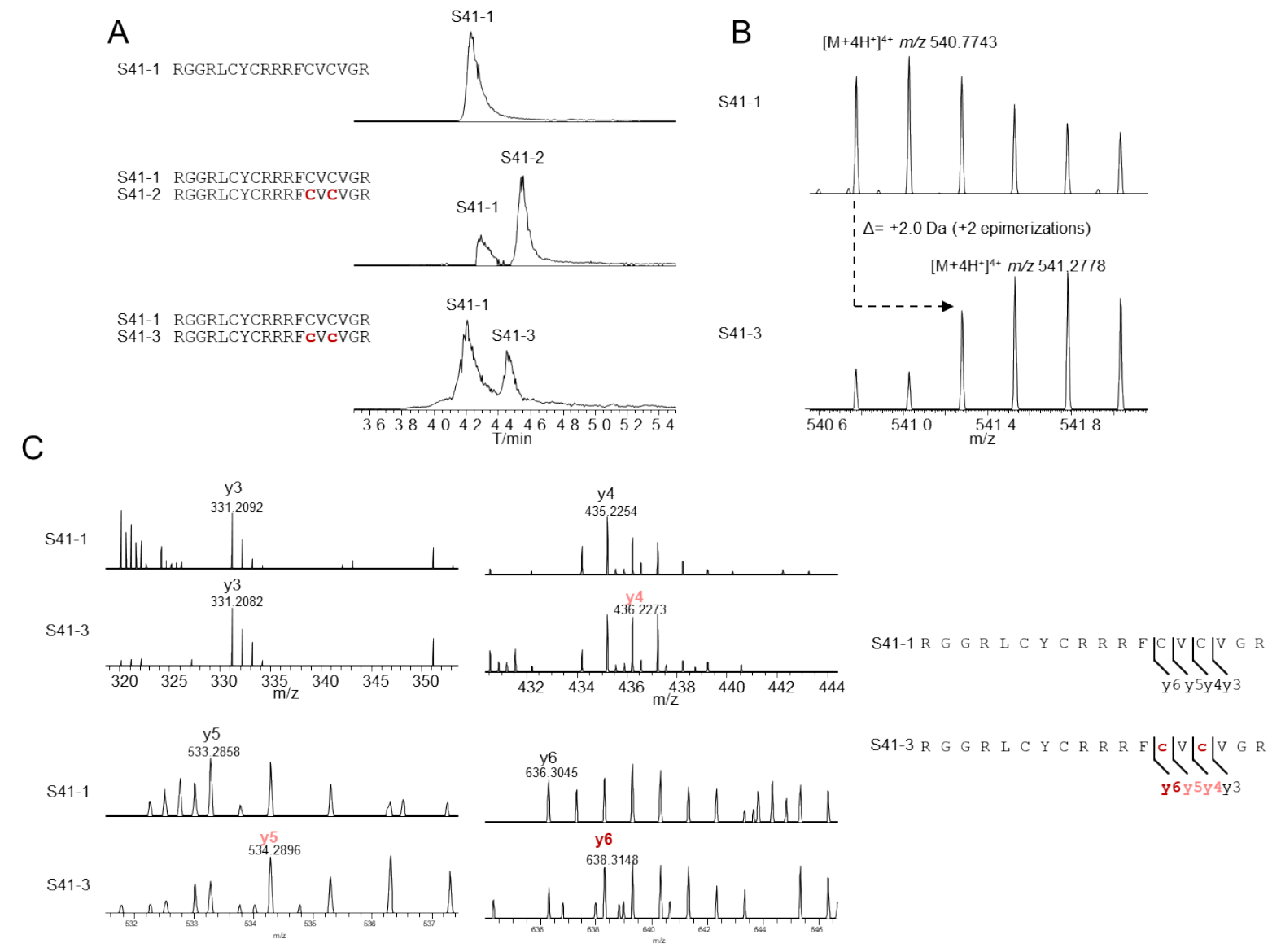

Figure S41. Characterization of selectively labeled products from co-expression of ospA-protegrin-1 + ospD using ODIS. His 6 -hybrid variant was cleaved with Factor Xa. (A) EICs of unmodified core peptides (S41-1), core peptides epimerized in TB (S41-2), and epimerized in $\mathrm{D}_{2} \mathrm{O}$-based medium (S41-3). (B) MS for parent ions of S41-1 and S41-3 acquired by LC-MS full-scan of ODIS experiment. (C) MS ${ }^{2}$ derived from parent ions of S41-1 and S41-3 acquired by LC-MS ${ }^{2}$ (PRM) of ODIS experiments. Bold (red) residues indicate epimerized residues. Small letter (red) indicates selective labeling with deuterium at the corresponding epimerized residue. Rose-colored b/y ions have mass shift of +1.0 Da, red b/y ions of +2.0 Da. 


\section{References}

(1) Cahn, J. K. B., Brinkmann-Chen, S., and Arnold, F. H. (2018) Enzyme nicotinamide cofactor specificity reversal guided by automated structural analysis and library design, Methods Mol. Biol. 1671, 15-26.

(2) Fuchs, S. W., Lackner, G., Morinaka, B. I., Morishita, Y., Asai, T., Riniker, S., and Piel, J. (2016) A Lanthipeptide - like N - terminal leader region guides peptide epimerization by radical SAM epimerases: implications for RiPP evolution, Angew. Chem. Int. Ed. 55, 12330-12333.

(3) Morinaka, B. I., Verest, M., Freeman, M. F., Gugger, M., and Piel, J. (2017) An orthogonal $\mathrm{D}_{2} \mathrm{O}$-based induction system that provides insights into D-amino acid pattern formation by radical S-adenosylmethionine peptide epimerases, Angew. Chem. Int. Ed. 56, $762-766$.

(4) Velappan, N., Sblattero, D., Chasteen, L., Pavlik, P., and Bradbury, A. R. M. (2007) Plasmid incompatibility: more compatible than previously thought?, Protein Eng. Des. Sel. 20, 309-313.

(5) Patiny, L., and Borel, A. (2013) ChemCalc: a building block for tomorrow's chemical infrastructure, J. Chem. Inf. Model. 53, $1223-1228$.

(6) Morinaka, B. I., Vagstad, A. L., Helf, M. J., Gugger, M., Kegler, C., Freeman, M. F., Bode, H. B., and Piel, J. (2014) Radical S-adenosyl methionine epimerases: regioselective introduction of diverse D-amino acid patterns into peptide natural products, Angew. Chem. Int. Ed. 53, 8503-8507.

\section{Author Contributions}

MK, SWF and JP designed the research; MK and JP wrote the manuscript and supporting information; MK, SWF, KF, CE and LVZ cloned, expressed and assayed the enzymes. 